\title{
On the Dirichlet problem for variational integrals in $B V$
}

\author{
By Lisa Beck at Pisa and Thomas Schmidt at Erlangen
}

\begin{abstract}
We investigate the Dirichlet problem for multidimensional variational integrals with linear growth which is formulated in a generalized way in the space of functions of bounded variation. We prove uniqueness of minimizers up to additive constants and deduce additional assertions about these constants and the possible (non-)attainment of the boundary values. Moreover, we provide several related examples. In the case of the model integral

$$
\int_{\Omega} \sqrt{1+|\nabla w|^{2}} d x \quad \text { for } w: \mathbb{R}^{n} \supset \Omega \rightarrow \mathbb{R}^{N}
$$

our results extend classical results from the scalar case $N=1$-where the problem coincides with the non-parametric least area problem - to the general vectorial setting $N \in \mathbb{N}$.
\end{abstract}

\section{Contents}

1. Introduction and statement of the main results

1.1. Graphs of least gradient

1.2. Variational integrals with linear growth

2. Notation and preliminaries

3. Various examples

3.1. Examples of integrands

3.2. Proof of Theorem 1.5

3.3. Santi's example, revisited

3.4. An $N$-parameter-family of minimizers

4. Local boundedness

5. Uniqueness

5.1. Regularization and approximation

5.2. Estimates for first and second derivatives

5.3. Proofs of the uniqueness results

5.4. The set of generalized minimizers 
6. Non-uniqueness and boundary behavior
A. (Semi-)Continuity and existence
B. Additional remarks on the Dirichlet problem
C. Non-autonomous integrals
D. Maximum principles
References

\section{Introduction and statement of the main results}

We consider multidimensional variational integrals

$$
F[w]:=\int_{\Omega} f(\nabla w) d x \quad \text { for } w: \Omega \rightarrow \mathbb{R}^{N}
$$

where throughout this paper $\Omega$ is a non-empty, bounded, open, and connected ${ }^{1)}$ subset of $\mathbb{R}^{n}(n \geqq 2)$ with Lipschitz boundary and $f: \mathbb{R}^{N n} \rightarrow[0, \infty)$ is a given integrand which will be specified below. We are interested in the minimization problem for $F$ in Dirichlet classes, and in particular in existence, uniqueness, and regularity of (generalized) minimizers. We stress that $N \in \mathbb{N}$ denotes an arbitrary natural number, and hence the problem is a vectorial one and is related to a system of partial differential equations.

1.1. Graphs of least gradient. Postponing the treatment of general integrands $f$ to Section 1.2 let us restrict the discussion to the model integrals

$$
E_{\lambda}[w]:=\int_{\Omega} e_{\lambda}(\nabla w) d x
$$

with the density ${ }^{2)}$

$$
e_{\lambda}(z):=\sqrt{\lambda^{2}+|z|^{2}}
$$

where $\lambda \geqq 0$ is a fixed parameter. These integrals can be understood as a limit case of the $p$-energies

$$
\int_{\Omega}\left(\lambda^{2}+|\nabla w|^{2}\right)^{\frac{p}{2}} d x
$$

where $p \geqq 1$ is another parameter. However, while for $p>1$ existence and uniqueness of minimizers can be obtained in the Sobolev space $W^{1, p}\left(\Omega, \mathbb{R}^{N}\right)$, in the case $p=1$ the application of the direct method generally fails in the non-reflexive Sobolev space $W^{1,1}\left(\Omega, \mathbb{R}^{N}\right)$, due to its lack of compactness properties. Existence results can rather be obtained in the space $B V\left(\Omega, \mathbb{R}^{N}\right)$ of functions of bounded variation. Precisely, one fixes a Dirichlet class

$$
\mathscr{D}=u_{0}+W_{0}^{1,1}\left(\Omega, \mathbb{R}^{N}\right)
$$

\footnotetext{
1) The assumption that $\Omega$ be connected is just made in order to simplify some statements. Indeed, if $\Omega$ is disconnected, our analysis works on the connected components of $\Omega$.

2) Throughout the article $|z|$ denotes the Hilbert-Schmidt norm of the matrix $z \in \mathbb{R}^{N n}$, that is, $|z|^{2}:=\sum_{\alpha=1}^{N} \sum_{i=1}^{n}\left(z_{i}^{\alpha}\right)^{2}$.
} 
with $u_{0} \in W^{1,1}\left(\Omega, \mathbb{R}^{N}\right)$ and extends $E_{\lambda}$ from $\mathscr{D}$ to $B V\left(\Omega, \mathbb{R}^{N}\right)$ by letting

$$
\mathscr{E}_{\lambda}^{\mathscr{D}}[w]:=\int_{\Omega} e_{\lambda}(\nabla w) d x+\left|D^{s} w\right|(\Omega)+\int_{\partial \Omega}\left|w-u_{0}\right| d \mathscr{H}^{n-1} \quad \text { for } w \in B V\left(\Omega, \mathbb{R}^{N}\right),
$$

where $D w=\nabla w \cdot \mathscr{L}^{n}+D^{s} w$ is the decomposition of the $\mathbb{R}^{N n}$-valued gradient measure $D w$ into its absolutely continuous and its singular part with respect to the Lebesgue measure $\mathscr{L}^{n}$. Moreover, $\left|D^{s} w\right|(\Omega)$ denotes the variation of $D^{s} w$ on $\Omega, \mathscr{H}^{n-1}$ the $(n-1)$-dimensional Hausdorff measure on $\mathbb{R}^{n}$, and the occurrence of $w$ and $u_{0}$ in the boundary integral is to be interpreted in the sense of trace ([40], [9]); see Section 2 for further details on terminology. The formula (1.3) has been proposed in [43], [7] and adapts classical ideas from [49] and the $B V$-theory of area minimizing hypersurfaces ([27], [65], [45], [66]); compare the monographs [37], [46], [44], [6]. Obviously, $\mathscr{E}_{\lambda}^{\mathscr{D}}[w]=E_{\lambda}[w]$ holds for $w \in \mathscr{D}$ and thus $\mathscr{E}_{\lambda}^{\mathscr{D}}$ is really an extension of $E_{\lambda}$. Moreover, building on results of [73] it was pointed out in [43] that $\mathscr{E}_{\lambda}^{\mathscr{D}}$ coincides with the extension by semicontinuity in the tradition of Lebesgue and Serrin [56], [78], [79], satisfies the relation

$$
\inf _{B V\left(\Omega, \mathbb{R}^{N}\right)} \mathscr{E}_{\lambda}^{\mathscr{D}}=\inf _{\mathscr{D}} E_{\lambda}
$$

and can be considered as the natural continuation of $E_{\lambda}$ outside $\mathscr{D}$. Thus, it is reasonable to give the following definition of generalized minimizers:

Definition 1.1 (generalized minimizer). A function $u \in B V\left(\Omega, \mathbb{R}^{N}\right)$ is called a generalized minimizer of $E_{\lambda}$ in $\mathscr{D}$ if

$$
\mathscr{E}_{\lambda}^{\mathscr{D}}[u] \leqq \mathscr{E}_{\lambda}^{\mathscr{D}}[w] \text { for all } w \in B V\left(\Omega, \mathbb{R}^{N}\right)
$$

We write $\mathscr{M}_{\lambda}^{\mathscr{D}}$ for the set of generalized minimizers of $E_{\lambda}$ in $\mathscr{D}$.

Generalized minimizers can alternatively be characterized ([43], [7], [46]) as the strong $L^{1}$-limits of minimizing sequences for $E_{\lambda}$ in $\mathscr{D}$ (see also Theorem 1.8). With this characterization at hand the existence problem can easily be solved: In fact, by Rellich's theorem in $B V$ there exists at least one generalized minimizer corresponding to each Dirichlet class $\mathscr{D}$ and hence the infimum on the left-hand side of (1.4) is a minimum.

It should be noted that Dirichlet classes are not closed in $B V\left(\Omega, \mathbb{R}^{N}\right)$ with respect to the strong $L^{1}$-topology (or the weak-*-topology of $B V$ ) and generalized minimizers do not necessarily attain the boundary values $u_{0}$. Thus, the boundary integral in (1.3) need not vanish for generalized minimizers, and we get the following interpretation of this integral as a penalization term: The non-attainment of the boundary values is not generally ruled out but will instead be penalized by an increase of energy.

In the completely degenerate case $\lambda=0$ generalized minimizers are called functions of least gradient and interesting results in the scalar case $N=1$ have been obtained; see for instance [71], [82]. Here we will not further pursue this issue, but we will deal with the case $^{3)} \lambda>0$. Still in the scalar case $N=1$ we note that $E_{1}[w]$ represents the $n$-dimensional

3) Once $\lambda$ is positive, its precise value is not important. Indeed, the problems corresponding to different positive values of $\lambda$ can be transformed into each other by multiplying all the functions under consideration with a positive constant. 
area of the graph of $w$, and thus the problem is a very classical one, namely the nonparametric least area problem. Its generalized minimizers are called area minimizing graphs or non-parametric area minimizing hypersurfaces. They are some of the most studied analytical objects in the last century, and we refer the reader to [64], [53], [25], [24], [80], [45], [66], [81] and to the monograph [46], where an exhaustive list of additional references can be found.

In contrast, for $N>1$ the integral $E_{\lambda}[w]$ is in general very different from the area of the graph of $w$. In fact, the area integral ${ }^{4)}$ involves all the minors of the gradient matrix while $E_{\lambda}$ just involves the modulus of this matrix, that is the first order minors. However, $E_{\sqrt{n}}[w]$ is the $L^{1}$-norm of the gradient of the graph mapping $x \mapsto(x, w(x))$ which motivates us to call the generalized minimizers of $E_{\sqrt{n}}$ graphs of least gradient. We will study uniqueness and regularity of these objects which will turn out to be quite delicately linked. The authors are aware of only two previous results concerning these topics in the vectorial setting. One result is the partial regularity theorem of Anzellotti and Giaquinta [10] (see also [18]) which gives $C_{\text {loc }}^{1, \alpha}$-regularity on an open subset of $\Omega$ with full Lebesgue measure. The other result, due to Bildhauer, concerns everywhere gradient regularity, and since it is closely related to our approach, we restate it in the present setting:

Theorem 1.2 (Bildhauer [15], [17]). Let $\lambda>0$ and suppose that there holds

$$
\text { either } n=2 \text { or } u_{0} \in L^{\infty}\left(\Omega, \mathbb{R}^{N}\right) \text {. }
$$

Then there exists a generalized minimizer $u$ of $E_{\lambda}$ in $\mathscr{D}$ which satisfies

$$
u \in W^{1,1}\left(\Omega, \mathbb{R}^{N}\right) \quad \text { and } \quad|\nabla u| \log \left(1+|\nabla u|^{2}\right) \in L_{\text {loc }}^{1}(\Omega) .
$$

Turning to uniqueness the reader should note that $\mathscr{E}_{\lambda}^{\mathscr{D}}$ is convex but not strictly convex. More precisely, for $\lambda>0$ the integrand $e_{\lambda}$ and the first term in (1.3) are strictly convex, but the other two terms are merely convex. Thus, uniqueness of generalized minimizers is not immediate, and there are two potential sources of non-uniqueness, namely the possible occurrence of singular parts of the derivative and the possible non-attainment of the boundary values. In this paper we will rule out the first source: We will prove that $u \in W^{1,1}\left(\Omega, \mathbb{R}^{N}\right)$ holds for every generalized minimizer $u$ and not just that there exists a suitable one as stated in Theorem 1.2. Moreover, we will remove the assumption (1.5). In this way we will establish the following theorem as a particular case of our main results (see Theorem 1.10, Corollary 1.13, and Theorem 1.16 for the general statements and Section 5 for the proofs).

Theorem 1.3 (uniqueness up to constants). Let $\lambda>0$. Then the generalized minimizers of $E_{\lambda}$ in $\mathscr{D}$ are unique up to additive constants, that is, for $u, v \in \mathscr{M}_{\lambda}^{\mathscr{D}}$ there exists a constant $y \in \mathbb{R}^{N}$ such that $u=v+y$ holds a.e. on $\Omega$. Furthermore, the minimizers form a 1-parameter family, and indeed $\mathscr{M}_{\lambda}^{\mathscr{D}}$ can be parametrized by a compact interval,

4) The non-parametric area integral in higher codimension $N>1$ is non-convex with a delicate degeneration structure. It behaves in many aspects different from $E_{\lambda}$ and is related to several challenging and unsolved problems. We refer to [44] for a detailed discussion, to [55] for counterexamples, and to [88], [1], [87], [86] for some results, relying on methods from topology and the parametric setting of geometric measure theory ([37]). 
precisely

$$
\mathscr{M}_{\lambda}^{\mathscr{D}}=\{\bar{u}+t \bar{y}: t \in[-1,1]\}
$$

for some $\bar{y} \in \mathbb{R}^{N}$ and a particular generalized minimizer $\bar{u}$.

We stress that in the generality of our setting one cannot expect to remove the second source of non-uniqueness mentioned above and thus cannot improve Theorem 1.3 to full uniqueness, not even in the case $n=2, N=1$ of two-dimensional area minimizing graphs. This is shown by a classical example of Santi [74]; see also [46], Example 15.12, Section 3.3 below, and [11]. The next theorem states that non-uniqueness happens only in quite particular situations and that Santi's scalar example is symptomatic even for the vectorial case.

Theorem 1.4 (non-uniqueness and boundary behavior). Let $\lambda>0$ and suppose that generalized minimizers of $E_{\lambda}$ in $\mathscr{D}$ are not unique, that is, $\mathscr{M}_{\lambda}^{\mathscr{D}}$ contains more than one element. Then for every $u \in \mathscr{M}_{\lambda}^{\mathscr{D}}$ the jump $u-u_{0}$ at the boundary takes values in the 1-dimensional subspace $\mathbb{R} \bar{y}$ a.e. on $\partial \Omega$. Moreover, $\partial \Omega$ can be decomposed into disjoint subsets $(\partial \Omega)_{-}$and $(\partial \Omega)_{+}$with

$$
\mathscr{H}^{n-1}\left((\partial \Omega)_{-}\right)=\mathscr{H}^{n-1}\left((\partial \Omega)_{+}\right)=\frac{1}{2} \mathscr{H}^{n-1}(\partial \Omega)
$$

such that

$$
\bar{u} \pm J \bar{y}=u_{0} \quad \mathscr{H}^{n-1} \text {-a.e. on }(\partial \Omega)_{ \pm}
$$

for some function $J: \partial \Omega \rightarrow \mathbb{R}$ with $^{5)}$

$$
\inf _{(\partial \Omega)_{-}} J=\inf _{(\partial \Omega)_{+}} J=1
$$

Here, $\bar{y}$ and $\bar{u}$ denote the constant and the particular minimizer introduced in the statement of Theorem 1.3.

Theorem 1.4 will be proved in Section 6 as a particular case of Theorem 1.17 and Remark 1.18 below.

Let us make a couple of related remarks. Primarily we mention that $\bar{y}$ is unique up to change of sign and-having assumed non-uniqueness of minimizers-different from 0 . Moreover, $\bar{u}$ is uniquely determined. Secondly we point out that the situation described in the theorem cannot occur and we must thus have full uniqueness if one of the following two assertions holds:

- The particular minimizer $\bar{u}$ (or some other minimizer apart from the extremal ones $\bar{u}+\bar{y}$ and $\bar{u}-\bar{y}$ ) attains the boundary values $u_{0}$ on a set of positive $\mathscr{H}^{n-1}$-measure.

- Some minimizer attains the boundary values $u_{0}$ on more than half the boundary.

5) The infima in Theorem 1.4 are essential infima with respect to $\mathscr{H}^{n-1}$. 
We stress that at this point we have already obtained a quite accurate description of (non-)uniqueness and (non-)attainment of the boundary values. In fact-apart from those already ruled out - every combination of these phenomena may occur; compare Proposition 6.4 and Remark 6.5.

At first glance one might wonder whether there is a counterpart of Theorem 1.4 for unique minimizers, or in other words for $\bar{y}=0$. While it is not clear what should be a reasonable analogon of (1.7), one might at least hope that $u-u_{0}$ still takes values in a 1-dimensional subspace. However, the following example shows that this is not true, and thus the non-uniqueness assumption in Theorem 1.4 cannot be removed.

Theorem 1.5 (an example of non-attainment). For $n=N=2$ consider the annulus

$$
\Omega=\left\{x \in \mathbb{R}^{2}: 1<|x|<2\right\}
$$

and the boundary datum $u_{0}$ given by

$$
u_{0}(x)= \begin{cases}M x & \text { for }|x|=1, \\ 0 & \text { for }|x|=2,\end{cases}
$$

where $M \in \mathbb{R}$ is a constant. Then there is a unique generalized minimizer $u$ of $E_{1}$ in $\mathscr{D}$ and there holds

$$
\sup _{\Omega}|u| \leqq \frac{2}{1-\log 2}
$$

In particular, for $|M|>2 /(1-\log 2)$ the values of $u-u_{0}$ on $\partial \Omega$ are not contained in any 1-dimensional subspace of $\mathbb{R}^{2}$.

Theorem 1.5 is a refinement of a well-known example for area minimizing graphs and will be established in Section 3.2.

1.2. Variational integrals with linear growth. In this subsection we generalize Theorem 1.3 and Theorem 1.4 and restate our results in the setting of (1.1) with a general integrand $f$. As in Section 1.1 the focus is on the vectorial case $N>1$, but nevertheless we believe that the general form of our results presented here is new and interesting even in the scalar case $N=1$.

Of course, we have to impose growth, coercivity, convexity, and structure conditions on $f$. To begin we just suppose that $f: \mathbb{R}^{N n} \rightarrow[0, \infty)$ is convex and has linear growth in the sense of

$$
\gamma|z| \leqq f(z) \leqq \Gamma(1+|z|) \quad \text { for all } z \in \mathbb{R}^{N n}
$$

with positive constants $\Gamma$ and $\gamma$. Then fixing a Dirichlet class

$$
\begin{array}{r}
\mathscr{D}=u_{0}+W_{0}^{1,1}\left(\Omega, \mathbb{R}^{N}\right) \\
\text { Bereitgestellt von | Universitaetsbibliothek Augsburg } \\
\text { Angemeldet } \\
\text { Heruntergeladen am | 04.02.19 08:18 }
\end{array}
$$


with $u_{0} \in W^{1,1}\left(\Omega, \mathbb{R}^{N}\right)$, we extend the integral $F$ in (1.1) from $\mathscr{D}$ to $B V\left(\Omega, \mathbb{R}^{N}\right)$ by letting

$$
\begin{aligned}
\mathscr{F}^{\mathscr{D}}[w]:= & \int_{\Omega} f(\nabla w) d x+\int_{\Omega} f^{\infty}\left(\frac{d D^{s} w}{d\left|D^{s} w\right|}\right) d\left|D^{s} w\right| \\
& +\int_{\partial \Omega} f^{\infty}\left(\left(u_{0}-w\right) \otimes v_{\Omega}\right) d \mathscr{H}^{n-1}
\end{aligned}
$$

for $w \in B V\left(\Omega, \mathbb{R}^{N}\right)$. This formula was introduced in [43], [7] (compare [8], [50] for a related notion of $B V$-solutions of elliptic equations), $v_{\Omega}$ denotes the outward unit normal vector to $\partial \Omega$ (which exists $\mathscr{H}^{n-1}$-a.e.), and

$$
f^{\infty}(z):=\lim _{s \rightarrow \infty} \frac{f(s z)}{s} \text { for } z \in \mathbb{R}^{N n}
$$

defines the recession function of $f$ which reflects the behavior of $f$ near infinity (see Section 2 for further terminology).

We extend Definition 1.1:

Definition 1.6 (generalized minimizer). Suppose that $f$ is convex with $(\mathrm{H} 1)$. A function $u \in B V\left(\Omega, \mathbb{R}^{N}\right)$ is called a generalized minimizer of $F$ in $\mathscr{D}$ if

$$
\mathscr{F}^{\mathscr{D}}[u] \leqq \mathscr{F}^{\mathscr{D}}[w] \text { for all } w \in B V\left(\Omega, \mathbb{R}^{N}\right)
$$

Definition 1.7 (minimizing sequence). Suppose that $f$ is convex with (H1). One says that a sequence $\left(u_{k}\right)_{k \in \mathbb{N}}$ in $\mathscr{D}$ is a minimizing sequence for $F$ in $\mathscr{D}$ if there holds

$$
F\left[u_{k}\right] \underset{k \rightarrow \infty}{\longrightarrow} \inf _{\mathscr{D}} F
$$

Next we state a characterization of generalized minimizers as limits of minimizing sequences. Basically, the result is well known (compare for instance [43], [7], [46], [5], [6]) but it seems that a precise proof which covers general integrands and Lipschitz domains has been written down only recently in [22], [16].

Theorem 1.8 (characterization of generalized minimizers). Suppose that $f$ is convex with $(\mathrm{H} 1)$. Then $u \in B V\left(\Omega, \mathbb{R}^{N}\right)$ is a generalized minimizer of $F$ in $\mathscr{D}$ if and only if there exists a minimizing sequence $\left(u_{k}\right)_{k \in \mathbb{N}}$ for $F$ in $\mathscr{D}$ such that $u_{k}$ converges to $u$ in $L^{1}\left(\Omega, \mathbb{R}^{N}\right)$. Moreover, one has

$$
\inf _{B V\left(\Omega, \mathbb{R}^{N}\right)} \mathscr{F}^{\mathscr{D}}=\inf _{\mathscr{D}} F .
$$

For convenience of the reader we outline a proof of Theorem 1.8 in Appendix A. By Rellich's theorem in $B V$ every minimizing sequence for $F$ in $\mathscr{D}$ has an $L^{1}$-convergent subsequence. Therefore, as an immediate consequence of Theorem 1.8 we get:

Corollary 1.9 (existence of generalized minimizers). Suppose that $f$ is convex with (H1). Then there exists a generalized minimizer of $F$ in $\mathscr{D}$ and hence the infimum on the left-hand side of (1.9) is a minimum. 
Now we state our main uniqueness result which will be proved in Section 5. It extends the $L \log L$-gradient regularity of [15] to all bounded minimizers, instead of only one, and gives in particular uniqueness of bounded minimizers. The boundedness assumption will be justified below.

Theorem 1.10 (uniqueness of bounded generalized minimizers). Assume that $f$ is a $C^{2}$-integrand which satisfies ${ }^{6)} f(0) \leqq \lambda,(\mathrm{H} 1)$ and

$$
\gamma(1+|z|)^{-3}|\tilde{z}|^{2} \leqq \nabla^{2} f(z)(\tilde{z}, \tilde{z}) \leqq \Gamma(1+|z|)^{-1}|\tilde{z}|^{2} \quad \text { for all } z, \tilde{z} \in \mathbb{R}^{N n}
$$

with the constants $\gamma$ and $\Gamma$ from $(\mathrm{H} 1)$. Then we have:

Regularity. Every generalized minimizer $u \in B V\left(\Omega, \mathbb{R}^{N}\right) \cap L^{\infty}\left(\Omega, \mathbb{R}^{N}\right)$ of $F$ satisfies

$$
u \in W^{1,1}\left(\Omega, \mathbb{R}^{N}\right) \quad \text { and } \quad|\nabla u| \log \left(1+|\nabla u|^{2}\right) \in L_{\text {loc }}^{1}(\Omega) .
$$

Moreover, for every ball $B_{2 r}\left(x_{0}\right) \subset \Omega$ there holds

$$
\int_{B_{r}\left(x_{0}\right)}|\nabla u| \log \left(1+|\nabla u|^{2}\right) d x \leqq C\left(\lambda+\frac{1}{r} \sup _{B_{2 r\left(x_{0}\right)}}|u|\right) \int_{B_{2 r}\left(x_{0}\right)}(1+|\nabla u|) d x
$$

with a positive constant $C$ depending only on $n, N, \gamma$, and $\Gamma$.

Uniqueness. Whenever $u, v \in B V\left(\Omega, \mathbb{R}^{N}\right) \cap L^{\infty}\left(\Omega, \mathbb{R}^{N}\right)$ are generalized minimizers of $F$ in $\mathscr{D}$, then there exists a constant $y \in \mathbb{R}^{N}$ such that $u=v+y$ holds a.e. on $\Omega$.

Let us mention that $(\mathrm{H} 2)$ is a particular case of the $\mu$-ellipticity condition

$$
\left(\mathrm{H} 2_{\mu}\right) \quad \gamma(1+|z|)^{-\mu}|\tilde{z}|^{2} \leqq \nabla^{2} f(z)(\tilde{z}, \tilde{z}) \leqq \Gamma(1+|z|)^{-1}|\tilde{z}|^{2} \quad \text { for all } z, \tilde{z} \in \mathbb{R}^{N n}
$$

where $\mu>1$ is a parameter and $\gamma$ and $\Gamma$ are positive constants. This condition allows that the dispersion (or ellipticity) ratio

$$
\Lambda_{f}(z):=\frac{\max _{|\tilde{z}|=1} \nabla^{2} f(z)(\tilde{z}, \tilde{z})}{\min _{|\tilde{z}|=1} \nabla^{2} f(z)(\tilde{z}, \tilde{z})},
$$

a crucial number for matters of regularity, may blow up, when $z$ approaches infinitywhich means that the problem is a degenerate one. Very similar degeneration phenomena have been studied in terms of Bernstein's genre ([13], [80]) and in connection with $(p, q)$ growth conditions ([57], [58], [59], [72], [33], [19], [34], [35], [60], [76], [77]) and the $L \log L-$ energy ([38], [63], [36]). In all these cases gradient regularity can still be obtained provided the blow-up is sufficiently slow. In particular, following previous developments in the $(p, q)$-context Bildhauer and Fuchs [14], [20], [15], [16] proposed a quite complete uniqueness and regularity theory for variational integrals with linear growth and $\mu$-ellipticity $\left(\mathrm{H} 2{ }_{\mu}\right)$

6) The requirement $f(0) \leqq \lambda$ is just imposed in order to record the dependence on $f(0)$ precisely. 
for $\mu<3$ (partially imposing the stronger bound $\mu<(n+2) / n$; compare also Marcellini and Papi [60]). The limit case $\mu=3$, however, is of particular interest since it is just the condition satisfied by the integrands $e_{\lambda}$ from Section 1.1 with $\lambda>0$ (in fact $\left.\Lambda_{e_{\lambda}}(z) \approx 1+|z|^{2}\right)$. While most of the theory fails in this case, some arguments have been adapted ([15], [17], [16]) yielding, as a special case, Theorem 1.2. It should be noted that for $\mu>3$ there is few hope for regularity (compare [23] and Remark C.2), and thus Theorem 1.10 deals with a borderline case.

The arguments employed for uniqueness in the case $\mu<3$ rely on everywhere $C^{1}$-regularity for (at least) one minimizer, and momentarily it seems quite hopeless to prove such a strong regularity assertion in the limit case $\mu=3$. Therefore, we rather base our proof of Theorem 1.10 on $W^{1,1}$-regularity for every minimizer. To this end we basically follow ideas from [15], but we use Ekeland's variational principle ([30], [31], [32]) to construct 'nice' minimizing sequences near a given minimizer. This approach first appeared in the context of regularity in [61], [39], [2], but as a novel feature we now apply the principle in the negative Sobolev space $W^{-1,1}$. Moreover, we employ a particular regularization procedure which is motivated by ideas of [26].

Next - as announced above-we will be concerned with the $L^{\infty}$-assumption. If the integrand has a particular structure, then boundedness of minimizers (or even of certain minimizing sequences) can be deduced from the boundedness of the boundary values by a maximum principle; see for instance [29], [33], [15], [21]. For the sake of completeness we shall discuss relevant versions of such principles in Appendix D. However, in the following we adopt a somewhat different strategy and we derive local boundedness by Moser's iteration technique ([68], [69]) as an interior regularity property of minimizers. This method requires a different structure condition but allows to remove the $L^{\infty}$-assumption in Theorem 1.10 without imposing a restriction on the boundary data.

Theorem 1.11 (local boundedness of generalized minimizers). Assume that $f$ is a convex $C^{1}$-integrand which satisfies $f(0) \leqq \lambda,(\mathrm{H} 1)$ and

$$
\left[\xi^{T} \nabla f(z)\right] \cdot\left[\xi^{T} z\right] \geqq-\lambda|\xi|^{2} \quad \text { for all } z \in \mathbb{R}^{N n}, \xi \in \mathbb{R}^{N}
$$

with a nonnegative constant $\lambda$. Then every generalized minimizer $u \in B V\left(\Omega, \mathbb{R}^{N}\right)$ of $F$ is locally bounded, i.e., $u \in L_{\mathrm{loc}}^{\infty}\left(\Omega, \mathbb{R}^{N}\right)$, and it satisfies for each pair $B_{r}\left(x_{0}\right) \subset B_{R}\left(x_{0}\right) \subset \Omega$ of concentric balls the estimate

$$
\sup _{B_{r}\left(x_{0}\right)}|u| \leqq \frac{C}{(R-r)^{n}} \int_{B_{R}\left(x_{0}\right)}[(R-r) \lambda+|u|] d x
$$

with a constant $C$ depending only on $n, N, \gamma$, and $\Gamma$.

Theorem 1.11 will be established in Section 4.

Remark 1.12. In principle, the convexity assumption in Theorem 1.11 is dispensable, and-employing an adequate version of Theorem 1.8 ([5], [6], [54]) - an extension to quasiconvex integrands $f$ is possible. Moreover, adopting notions of generalized minimizers as in [75] one might handle even non-quasiconvex situations. However, convexity 
seems to be inevitable for the other results of this paper, and thus we do not further pursue these generalizations.

We combine Theorem 1.10 and Theorem 1.11 getting (see Section 5.3 for a detailed proof):

Corollary 1.13 (uniqueness of generalized minimizers). Assume that $f$ is a $C^{2}$ integrand which satisfies the assumptions $(\mathrm{H} 1),(\mathrm{H} 2)$, and $(\mathrm{H} 3)$. Then generalized minimizers of $F$ in $\mathscr{D}$ are unique in $B V\left(\Omega, \mathbb{R}^{N}\right)$ up to additive constants. Furthermore, each such minimizer $u \in B V\left(\Omega, \mathbb{R}^{N}\right)$ satisfies

$$
u \in W^{1,1}\left(\Omega, \mathbb{R}^{N}\right) \quad \text { and } \quad|\nabla u| \log \left(1+|\nabla u|^{2}\right) \in L_{\text {loc }}^{1}(\Omega),
$$

and for every ball $B_{3 r}\left(x_{0}\right) \subset \Omega$ there holds the estimate

$$
\frac{1}{r^{n}} \int_{B_{r}\left(x_{0}\right)}|\nabla u| \log \left(1+|\nabla u|^{2}\right) d x \leqq C\left(1+\frac{1}{r^{n}} \int_{B_{3 r}\left(x_{0}\right)}|\nabla u| d x\right)^{2}
$$

with a positive constant $C$ depending only on $n, N, \gamma, \Gamma$, and $\lambda$.

Next let us discuss the mild structure condition (H3), which can be rewritten in coordinates as

$$
\sum_{i=1}^{n} \sum_{\alpha, \beta=1}^{N} \xi^{\alpha} \frac{\partial f}{\partial z_{i}^{\alpha}}(z) z_{i}^{\beta} \xi^{\beta} \geqq-\lambda|\xi|^{2}
$$

Thus, for $N=1$ assumption $(\mathrm{H} 3)$ reduces to $\nabla f(z) \cdot z \geqq-\lambda$ and is automatically satisfied by Lemma 2.8 . This is not surprising since both maximum principles and $L^{\infty}$-regularity are usually valid in the scalar case without particular hypotheses on the structure of the integrand. In contrast, in the vectorial case it is known by a series of striking examples [28], [48], [70], [52], [83], [84] that one cannot hope for everywhere regularity, not even for boundedness of minimizers without some kind of particular structure. A sufficient structure for regularity, namely rotational symmetry $f(z)=\tilde{f}(|z|)$, has first been identified by Uhlenbeck in connection with the interior $C^{1, \alpha}$-regularity for minimizers of the $p$-energies (1.2); see [85], [42], [3], [51], [60]. However, here we are just interested in boundedness of minimizers, and thus the weaker structure condition (H3) suffices; see Meier [62] for the first occurrence of a similar lower bound. Another reasonable condition occurring in [29], [33], [21] is

$$
f(z)=\tilde{f}\left(\left|z_{1}\right|,\left|z_{2}\right|, \ldots,\left|z_{n}\right|\right)
$$

where $z_{i} \in \mathbb{R}^{N}$ denotes the $i$ th column of the matrix $z \in \mathbb{R}^{N n}$. In our context $(1.10)$ is interesting since it provides a simple sufficient criterion for (H3). More precisely, for a $C^{1}$-integrand $f: \mathbb{R}^{N n} \rightarrow \mathbb{R}$ we have

$f$ is convex with $(1.10)$

$$
\begin{aligned}
& \Rightarrow \quad f \text { satisfies }(1.10) \text { and } \tilde{f}:[0, \infty)^{n} \rightarrow \mathbb{R} \text { is non-decreasing in each argument } \\
& \Rightarrow \quad f \text { satisfies }(\mathrm{H} 3) \text { with } \lambda=0 .
\end{aligned}
$$


To derive some consequences of uniqueness up to constants we shall have a closer look at the recession function $f^{\infty}$. We start noting that in our setting $f^{\infty}$ is positive on $\mathbb{R}^{N n} \backslash\{0\}, 1$-homogeneous, and convex; in other words $f^{\infty}$ is a norm on $\mathbb{R}^{N n}$. We shall need an additional assumption which is related to strict convexity of $f^{\infty}$. However, since $f^{\infty}$ is 1-homogeneous, it can obviously not be strictly convex in the radial directions. Therefore we use the following concept.

Definition 1.14 (strict convexity of norms). Let $m \in \mathbb{N}$. A norm $g$ on $\mathbb{R}^{m}$ is said to be a strictly convex norm if its unit ball is strictly convex, that is, if equality

$$
g\left(y_{1}\right)=g\left(y_{2}\right)=g\left(\lambda y_{1}+(1-\lambda) y_{2}\right)=1 \quad \text { with } \lambda \in(0,1), y_{1}, y_{2} \in \mathbb{R}^{m}
$$

already implies

$$
y_{1}=y_{2}
$$

In the following it will be convenient to consider for $0 \neq v \in \mathbb{R}^{n}$ the norm $y \mapsto f^{\infty}(y \otimes v)$ on $\mathbb{R}^{N}$. We will suppose:

$$
\begin{gathered}
\text { For every } 0 \neq v \in \mathbb{R}^{n} \text { the map } \\
y \mapsto f^{\infty}(y \otimes v) \text { is a strictly convex norm on } \mathbb{R}^{N} .
\end{gathered}
$$

We stress that (H4) can in general not be deduced from strict convexity of $f$ (see Remark 3.3). Nevertheless, the following simple criteria justify the introduction of (H4).

Remark 1.15. Suppose that $f$ is convex with (H1). Then (H4) is implied by each of the following conditions:

- $f^{\infty}$ itself is a strictly convex norm or

- $f$ satisfies $^{7)}$ the structure condition (1.10).

Next we deal with the remaining assertions of Theorem 1.3 and Theorem 1.4 and we restate them in a more general context.

Theorem 1.16 (the set of generalized minimizers). Suppose that $f$ is convex with (H1). Moreover, suppose that generalized minimizers of $F$ in $\mathscr{D}$ are unique up to additive constants and that $f^{\infty}$ satisfies (H4). Then the set of all generalized minimizers of $F$ in $\mathscr{D}$ can be written in the form

$$
\{\bar{u}+t \bar{y}: t \in[-1,1]\}
$$

with some constant $\bar{y} \in \mathbb{R}^{N}$ and some particular minimizer $\bar{u}$.

Theorem 1.16 will be proved in Section 5.4. Moreover, by means of a counterexample in Section 3.4 we demonstrate that $(\mathrm{H} 4)$ is indeed mandatory in Theorem 1.16.

7) It suffices to require somewhat less, namely that $f^{\infty}$ instead of $f$ has the structure in (1.10). 
Now we provide a statement about the boundary behavior of non-unique minimizers, which we will establish in Section 6.

Theorem 1.17 (non-uniqueness and boundary behavior). Suppose that we are in the situation of Theorem 1.16 with $\bar{y} \neq 0$. Then $\partial \Omega$ can be decomposed into disjoint subsets $(\partial \Omega)_{-}$and $(\partial \Omega)_{+}$such that

$$
\bar{u} \pm J \bar{y}=u_{0} \quad \mathscr{H}^{n-1} \text {-a.e. on }(\partial \Omega)_{ \pm}
$$

for some function $J: \partial \Omega \rightarrow \mathbb{R}$ with

$$
\inf _{(\partial \Omega)_{-}} J=\inf _{(\partial \Omega)_{+}} J=1
$$

Moreover, there holds

$$
\int_{(\partial \Omega)_{-}} f^{\infty}\left(-\bar{y} \otimes v_{\Omega}\right) d \mathscr{H}^{n-1}=\int_{(\partial \Omega)_{+}} f^{\infty}\left(\bar{y} \otimes v_{\Omega}\right) d \mathscr{H}^{n-1}
$$

Here, the condition (1.11) implies some inequalities for the size of the sets $(\partial \Omega)_{-}$and $(\partial \Omega)_{-}$, namely

$$
\frac{\min _{|z|=1} f^{\infty}(z)}{\max _{|z|=1} f^{\infty}(z)} \leqq \frac{\mathscr{H}^{n-1}\left((\partial \Omega)_{-}\right)}{\mathscr{H}^{n-1}\left((\partial \Omega)_{+}\right)} \leqq \frac{\max _{|z|=1} f^{\infty}(z)}{\min _{|z|=1} f^{\infty}(z)}
$$

but in general the identity (1.11) does not give a more precise information; compare Proposition 6.4. However, imposing another structure condition-which is in some sense the opposite of (1.10) - we come out with the simple assertion (1.6), which we previously had in Theorem 1.4.

Remark 1.18. Suppose that we are in the situation of Theorem 1.17 and additionally assume that $f$ satisfies $^{8)}$ the structure condition

$$
f(z)=\tilde{f}\left(\left|z^{1}\right|,\left|z^{2}\right|, \ldots,\left|z^{N}\right|\right)
$$

where $z^{\alpha}$ denotes the $\alpha$ th row of the matrix $z \in \mathbb{R}^{N n}$. Then there holds

$$
\mathscr{H}^{n-1}\left((\partial \Omega)_{-}\right)=\mathscr{H}^{n-1}\left((\partial \Omega)_{+}\right)=\frac{1}{2} \mathscr{H}^{n-1}(\partial \Omega) .
$$

Proof. As a consequence of (1.12) the recession function $f^{\infty}$ can be written as

$$
f^{\infty}(z)=(\tilde{f})^{\infty}\left(\left|z^{1}\right|,\left|z^{2}\right|, \ldots,\left|z^{N}\right|\right)
$$

8) Remark 1.18 still holds if instead of (1.12) for $f$ only the analogous structure condition for $f^{\infty}$ —as it occurs in the proof-is required. 
Consequently, we have

$$
f^{\infty}\left( \pm \bar{y} \otimes v_{\Omega}\right)=(\tilde{f})^{\infty}\left(\left|\bar{y}^{1}\right|,\left|\bar{y}^{2}\right|, \ldots,\left|\bar{y}^{N}\right|\right)
$$

the integrands in (1.11) are both constant with the same value, and (1.11) reduces to (1.13).

To finish this introductory exposition let us comment on two problems which seem to be open even for the model integral $E_{1}$ in the vectorial case.

The first problem is to determine what can be said-beyond the $L \log L$-regularity discussed in this paper-about the gradient of a generalized minimizer $u$ of $E_{1}$. To be more specific we would like to know whether necessarily $|\nabla u| \in L_{\text {loc }}^{1+\varepsilon}(\Omega)$ holds for some $\varepsilon>0$. Indeed, this question seems to be open even for $n=N=2$. However, while this is a delicate problem for $E_{1}$ and the limit case $\mu=3$ in $(\mathrm{H} 2 \mu), \nabla u$ is known to be Hölder continuous ([15], [20]) in the case $\mu<3$.

The second issue is boundary regularity of generalized minimizers. The above results describe the boundary behavior for general domains $\Omega$ and general boundary values $u_{0}$. However, imposing additional assumptions on $\Omega$ and $u_{0}$ one may hope to obtain stronger results, namely boundary regularity, attainment of the boundary values, and full uniqueness. For area minimizing graphs in the scalar case $N=1$ this program has been carried out in classical literature; see [66], [67], [81] as well as [46], Chapter 15, and the references quoted there. It is not clear to us whether these results or the relevant methods can be extended to the vectorial setting.

\section{Notation and preliminaries}

We briefly recall our global assumptions, imposed for the whole paper. As already mentioned in the introduction we fix $n, N \in \mathbb{N}$ (with $n \geqq 2$ ) and by $\Omega$ we always denote a non-empty, bounded, open, and connected subset of $\mathbb{R}^{n}$. Moreover, we generally assume that $\Omega$ has a Lipschitz boundary, that is, for every $x \in \partial \Omega$ there exists a bi-Lipschitz transformation $\Phi$ which maps the unit ball $B_{1}$ in $\mathbb{R}^{n}$ to a neighborhood of $x$ in such a way that for $y=\left(y_{1}, y_{2}, \ldots, y_{n}\right) \in B_{1}$ one has

$$
\begin{aligned}
& y_{n}>0 \Leftrightarrow \Phi(y) \in \Omega, \\
& y_{n}=0 \quad \Leftrightarrow \Phi(y) \in \partial \Omega, \\
& y_{n}<0 \quad \Leftrightarrow \quad \Phi(y) \notin \bar{\Omega} .
\end{aligned}
$$

Next we explain some general terminology and then we start collecting a couple of basic definitions and preliminary results.

Constants. We use various constants which are mostly understood to be positive and we generally indicate small constants by lowercase letters and large constants by uppercase letters. In particular, we write $c$ or $C$ for generic, positive constants which may vary from line to line and need not be the same in any two occurrences. 
Balls, boundary, closure. By $B_{r}^{n}\left(x_{0}\right)$ we abbreviate the open ball in $\mathbb{R}^{n}$ with center $x_{0}$ and radius $r$, that is, $B_{r}^{n}\left(x_{0}\right)=\left\{x \in \mathbb{R}^{n}:\left|x-x_{0}\right|<r\right\}$. The upper index ${ }^{n}$ will mostly be omitted when the context is unambiguous. Moreover, if $S$ is a set in $\mathbb{R}^{n}$, then we denote by $\partial S$ its topological boundary and by $\bar{S}$ its closure.

Measures, integration, function spaces, suprema, infima. Our terminology in this regard is mostly in accordance with [6] and some of it is explained after formula (1.3). Here, we just mention a few additional points: We write $w \cdot v$ for the weighted measure with weight $w$ and basic measure $v$, and we define the class $L_{M}^{\infty}\left(\Omega, \mathbb{R}^{N}\right)$ (with a positive constant $M)$ by letting

$$
L_{M}^{\infty}\left(\Omega, \mathbb{R}^{N}\right):=\left\{u \in L^{\infty}\left(\Omega, \mathbb{R}^{N}\right): \sup _{\Omega}|u| \leqq M\right\},
$$

where we used sup for the essential supremum with respect to the Lebesgue measure $\mathscr{L}^{n}$. In the following we will use inf and sup for essential infima and suprema with respect to either $\mathscr{L}^{n}$ or $\mathscr{H}^{n-1}$, depending on the context. Next we state a simple lemma involving the supnorm (see for instance [4], Theorem 2.14).

Lemma 2.1. For any Lebesgue-measurable function $w: \Omega \rightarrow \mathbb{R}^{N}$ there holds

$$
\sup _{\Omega}|w|=\lim _{p \rightarrow \infty}\left(\int_{\Omega}|w|^{p} d x\right)^{\frac{1}{p}}
$$

where the limit on the right-hand side exists in $[0, \infty]$.

The negative Sobolev space $\boldsymbol{W}^{-1,1}$. The negative Sobolev spaces $W^{-1, p}$ with $p>1$ are commonly viewed as the dual spaces of $W_{0}^{1, p /(p-1)}$. In contrast, the space $W^{-1,1}$ cannot be approached via duality and rarely occurs in the literature at all. Therefore, we now briefly review what is relevant for our purposes.

We introduce $W^{-1,1}\left(\Omega, \mathbb{R}^{N}\right)$ as the collection of all $\mathbb{R}^{N}$-valued distributions $T$ on $\Omega$ which can be written as

$$
T=w_{0}+\sum_{s=1}^{n} \partial^{s} w_{s}
$$

with $w_{0}, w_{1}, \ldots, w_{n} \in L^{1}\left(\Omega, \mathbb{R}^{N}\right)$. One easily checks that $W^{-1,1}\left(\Omega, \mathbb{R}^{N}\right)$ is a normed linear space if we let

$$
\|T\|_{W^{-1,1}\left(\Omega, \mathbb{R}^{N}\right)}:=\inf \sum_{s=0}^{n} \int_{\Omega}\left|w_{s}\right| d x \quad \text { for } T \in W^{-1,1}\left(\Omega, \mathbb{R}^{N}\right)
$$

where the infimum runs over all functions $w_{0}, w_{1}, \ldots, w_{n}$ representing $T$ as before.

To see that $W^{-1,1}\left(\Omega, \mathbb{R}^{N}\right)$ is complete and thus a Banach space we argue as follows. We consider the linear map

$$
P:\left[L^{1}\left(\Omega, \mathbb{R}^{N}\right)\right]^{1+n} \rightarrow W^{-1,1}\left(\Omega, \mathbb{R}^{N}\right), \quad\left(w_{0}, w_{1}, \ldots, w_{n}\right) \mapsto w_{0}+\sum_{s=1}^{n} \partial^{s} w_{s}
$$


and its factorial map

$$
J:\left[L^{1}\left(\Omega, \mathbb{R}^{N}\right)\right]^{1+n} / \operatorname{ker} P \rightarrow W^{-1,1}\left(\Omega, \mathbb{R}^{N}\right) .
$$

From the above definition of $W^{-1,1}$ we then infer that $J$ is onto and isometric ${ }^{9)}$. Hence $W^{-1,1}\left(\Omega, \mathbb{R}^{N}\right)$ is isometrically isomorphic to $\left[L^{1}\left(\Omega, \mathbb{R}^{N}\right)\right]^{1+n} / \operatorname{ker} P$. The latter space is a factor space of Banach spaces and thus is again a Banach space; see [89], Chapter I.11. In conclusion, $W^{-1,1}\left(\Omega, \mathbb{R}^{N}\right)$ is complete.

Moreover, we record the following inequalities for $w \in L^{1}\left(\Omega, \mathbb{R}^{N}\right)$ which are immediate by the above definition of $W^{-1,1}$ and its norm:

$$
\begin{aligned}
\|w\|_{W^{-1,1}\left(\Omega, \mathbb{R}^{N}\right)} & \leqq \int_{\Omega}|w| d x, \\
\left\|\partial^{S} w\right\|_{W^{-1,1}\left(\Omega, \mathbb{R}^{N}\right)} & \leqq \int_{\Omega}|w| d x .
\end{aligned}
$$

The space $\boldsymbol{B} \boldsymbol{V}$ of functions of bounded variation. The space $B V\left(\Omega, \mathbb{R}^{N}\right)$ is of substantial importance for this paper. By definition a function $w \in L^{1}\left(\Omega, \mathbb{R}^{N}\right)$ is in $B V\left(\Omega, \mathbb{R}^{N}\right)$ if the distributional derivative of $w$ can be represented by a finite Radon measure, which we then call $D w$. Moreover, by $D^{s} w$ we denote the singular part in the Lebesgue decomposition of $D w$ with respect to $\mathscr{L}^{n}$ and we write $\nabla w$ for the density of the absolutely continuous part. We refer-once more - to [6] for further information and proceed recalling the notion of strict convergence.

Definition 2.2 (strict convergence of measures and $B V$-functions). Consider a sequence $\left(\mu_{k}\right)_{k \in \mathbb{N}}$ of finite $\mathbb{R}^{m}$-valued Radon measures on $\Omega$ and a finite $\mathbb{R}^{m}$-valued Radon measure $\mu$ on $\Omega$. We say that $\mu_{k}$ converges strictly to $\mu$ on $\Omega$ if $\mu_{k}$ converges weakly-* to $\mu$ on $\Omega$ and if additionally there holds

$$
\left|\mu_{k}\right|(\Omega) \underset{k \rightarrow \infty}{\longrightarrow}|\mu|(\Omega)
$$

Moreover, we say that a sequence $\left(w_{k}\right)_{k \in \mathbb{N}}$ in $B V\left(\Omega, \mathbb{R}^{N}\right)$ converges strictly to some $w \in B V\left(\Omega, \mathbb{R}^{N}\right)$ if it converges in $L^{1}\left(\Omega, \mathbb{R}^{N}\right)$ and if moreover $D w_{k}$ converges strictly to $D w$ in the sense of measures.

The next lemma on approximations in $B V$ is similar to lemmas in [9], [10], [8]. The precise statement is taken from [16], Lemma B.1.

Lemma 2.3. For each $u \in B V\left(\Omega, \mathbb{R}^{N}\right)$ there exists a sequence $\left(w_{k}\right)_{k \in \mathbb{N}}$ in $W^{1,1}\left(\Omega, \mathbb{R}^{N}\right)$ with the following properties:

- Each $w_{k}$ coincides with $u$ on $\partial \Omega$ in the sense of trace.

- $w_{k}$ converges to $u$ in $L^{1}\left(\Omega, \mathbb{R}^{N}\right)$.

9) The factor space $\left[L^{1}\left(\Omega, \mathbb{R}^{N}\right)\right]^{1+n} / \operatorname{ker} P$ is endowed with the quotient norm. 

on $\Omega$.

- $\left(\mathscr{L}^{n}, D w_{k}\right)$ converges strictly to $\left(\mathscr{L}^{n}, D u\right)$ in the sense of $\mathbb{R}^{N n+1}$-valued measures

The recession function. For a convex function $f: \mathbb{R}^{m} \rightarrow[0, \infty)$ the recession function $f^{\infty}$ of $f$ is defined by

$$
f^{\infty}(z):=\lim _{s \rightarrow \infty} \frac{f(s z)}{s} \quad \text { for } z \in \mathbb{R}^{m}
$$
Here, by the convexity of $f$ the expression $\frac{f(s z)-f(0)}{s}$ is non-decreasing in $s$; thus the
limit in (2.4) always exists in $[0, \infty]$, satisfies

$$
f^{\infty}(z) \geqq f(z)-f(0),
$$

and $f^{\infty}$ is a well-defined, 1 -homogeneous function $\mathbb{R}^{m} \rightarrow[0, \infty]$ with possibly infinite values. Moreover, from the convexity of $f$ it follows that $f^{\infty}$ is lower semicontinuous and convex. If $f$ satisfies the right-hand inequality in $(\mathrm{H} 1)$, then $f^{\infty}$ is finite-valued, and if $f$ additionally satisfies the left-hand inequality in (H1), then $f^{\infty}$ is positive on $\mathbb{R}^{m} \backslash\{0\}$ and defines a norm on $\mathbb{R}^{m}$.

(Semi-)Continuity. Next we recall the (semi-)continuity theorem of Reshetnyak [73], which is a main ingredient in the proof of Theorem 1.8.

Theorem 2.4. Consider a sequence $\left(\mu_{k}\right)_{k \in \mathbb{N}}$ of finite $\mathbb{R}^{m}$-valued Radon measures on $\Omega$ which converges weakly-* to a finite $\mathbb{R}^{m}$-valued Radon measure $\mu$ on $\Omega$. Moreover, assume that all measures $\mu_{k}$ and $\mu$ take values ${ }^{10)}$ in some closed convex cone $K$ in $\mathbb{R}^{m}$.

Semicontinuity part. If $\bar{f}: K \rightarrow[0, \infty]$ is a lower semicontinuous, convex, and 1-homogeneous function, then there holds

$$
\int_{\Omega} \bar{f}\left(\frac{d \mu}{d|\mu|}\right) d|\mu| \leqq \liminf _{k \rightarrow \infty} \int_{\Omega} \bar{f}\left(\frac{d \mu_{k}}{d\left|\mu_{k}\right|}\right) d\left|\mu_{k}\right|
$$

Continuity part. If $\mu_{k}$ converges strictly to $\mu$ on $\Omega$ and $\bar{f}: K \rightarrow[0, \infty)$ is continuous and 1-homogeneous, then there holds

$$
\int_{\Omega} \bar{f}\left(\frac{d \mu}{d|\mu|}\right) d|\mu|=\lim _{k \rightarrow \infty} \int_{\Omega} \bar{f}\left(\frac{d \mu_{k}}{d\left|\mu_{k}\right|}\right) d\left|\mu_{k}\right| .
$$

Proof. The claims follow from Theorem 2.38 and Theorem 2.39 in [6] once one extends $f$ to all of $\mathbb{R}^{m}$ preserving the above assumptions. For the semicontinuity part such an extension is obtained by letting $\bar{f} \equiv \infty$ outside $K$; for the continuity part one extends $\bar{f}$ as a continuous, 1-homogeneous function.

10) By definition $\mu$ takes values in $K$ if $\mu(B) \in K$ holds for every Borel subset $B$ of $\Omega$. In this case the density $\frac{d \mu}{d|\mu|}$ also takes values in $K$. 
Remark 2.5. Following [43] in our applications of Theorem 2.4 we will mostly consider the function $\bar{f}: K \rightarrow[0, \infty]$ defined on the half-space $K=[0, \infty) \times \mathbb{R}^{N n}$ by

$$
\bar{f}(t, z):= \begin{cases}t f(z / t) & \text { for } t>0 \\ f^{\infty}(z) & \text { for } t=0\end{cases}
$$

where $f: \mathbb{R}^{N n} \rightarrow[0, \infty)$ is the integrand in (1.1). If $f$ is convex, then $\bar{f}$ is well-defined, lower semicontinuous, convex, and 1-homogeneous and moreover for all $w \in B V\left(\Omega, \mathbb{R}^{N}\right)$ there holds

$$
\int_{\Omega} \bar{f}\left(\frac{d\left(\mathscr{L}^{n}, D w\right)}{d\left|\left(\mathscr{L}^{n}, D w\right)\right|}\right) d\left|\left(\mathscr{L}^{n}, D w\right)\right|=\int_{\Omega} f(\nabla w) d x+\int_{\Omega} f^{\infty}\left(\frac{d D^{s} w}{d\left|D^{s} w\right|}\right) d\left|D^{s} w\right|
$$

Consequently, the semicontinuity part of Theorem 2.4 applies to the functional in (2.7) and a sequence $\left(w_{k}\right)_{k \in \mathbb{N}}$ in $B V\left(\Omega, \mathbb{R}^{N}\right)$ if $D w_{k}$ converges weakly-* in the sense of measures. If additionally (H1) holds, then $\bar{f}$ is finite-valued and continuous. Thus, also the continuity part of Theorem 2.4 applies if $\left(\mathscr{L}^{n}, D w_{k}\right)$ converges strictly in the sense of measures as in Lemma 2.3.

Next we state another semicontinuity result, tailored out for an application in Section 5.1, which we derive as a particular case of Theorem 2.4. that

Lemma 2.6. Let $p>1$ and suppose that $f: \mathbb{R}^{N n} \rightarrow[0, \infty)$ is a convex function such

$$
f(z) \geqq \gamma|z|^{p} \quad \text { for all } z \in \mathbb{R}^{N n}
$$

with some positive constant $\gamma$. Moreover, suppose that $g: \mathbb{R}^{N} \rightarrow[0, \infty)$ is a lower semicontinuous function and that $u_{0} \in W^{1, p}\left(\Omega, \mathbb{R}^{N}\right)$ is given, and let $\mathscr{D}^{p}:=u_{0}+W_{0}^{1, p}\left(\Omega, \mathbb{R}^{N}\right)$. Then the functional $F: W^{-1,1}\left(\Omega, \mathbb{R}^{N}\right) \rightarrow \mathbb{R}$ defined by

$$
F[w]:= \begin{cases}\int_{\Omega} f(\nabla w) d x+\int_{\Omega} g(w) d x & \text { for } w \in \mathscr{D}^{p} \\ \infty & \text { for } w \in W^{-1,1}\left(\Omega, \mathbb{R}^{N}\right) \backslash \mathscr{D}^{p}\end{cases}
$$

is lower semicontinuous with respect to convergence in the norm of $W^{-1,1}\left(\Omega, \mathbb{R}^{N}\right)$.

Proof. It suffices to prove $F[w] \leqq \lim _{k \rightarrow \infty} F\left[w_{k}\right]$ whenever $w_{k} \in \mathscr{D}^{p}$ converges to $w \in W^{-1,1}\left(\Omega, \mathbb{R}^{N}\right)$ and $\lim _{k \rightarrow \infty} F\left[w_{k}\right]$ exists in $[0, \infty)$. In this situation we exploit the lower bound on $f$ to deduce that $\left(\nabla w_{k}\right)_{k \in \mathbb{N}}$ is bounded in $L^{p}\left(\Omega, \mathbb{R}^{N n}\right)$. By Poincaré's inequality $\left(w_{k}\right)_{k \in \mathbb{N}}$ is bounded in $W^{1, p}\left(\Omega, \mathbb{R}^{N}\right)$, and a subsequence $\left(w_{k_{l}}\right)_{l \in \mathbb{N}}$ converges weakly in $W^{1, p}\left(\Omega, \mathbb{R}^{N}\right)$. The limit with respect to this convergence must be $w$, and since $\mathscr{D}^{p}$ is weakly closed in $W^{1, p}\left(\Omega, \mathbb{R}^{N}\right)$, also $w$ is in $\mathscr{D}^{p}$. Passing to another subsequence we may assume convergence $w_{k_{l}} \rightarrow w$ a.e., and moreover it follows that $D w_{k_{l}}$ converges to $D w$ weakly-* in the sense of measures on $\Omega$. Thus we may apply the semicontinuity part of Theorem 2.4 as explained in Remark 2.5 (note that in the present situation the involved measures are absolutely continuous and thus the last term in (2.7) vanishes) and Fatou's lemma getting 


$$
\begin{aligned}
F[w] & =\int_{\Omega} f(\nabla w) d x+\int_{\Omega} g(w) d x \\
& \leqq \liminf _{l \rightarrow \infty} \int_{\Omega} f\left(\nabla w_{k_{l}}\right) d x+\liminf _{l \rightarrow \infty} \int_{\Omega} g\left(w_{k_{l}}\right) d x \leqq \lim _{k \rightarrow \infty} F\left[w_{k}\right]
\end{aligned}
$$

Convex integrands. Next we deal with elementary properties of convex integrands $f$. We state two lemmas which provide upper and lower bounds for $\nabla f$. The first lemma is a particular case of [47], Lemma 5.2.

Lemma 2.7. Suppose that $f: \mathbb{R}^{m} \rightarrow[0, \infty)$ is a convex function with

$$
f(z) \leqq \Gamma(1+|z|) \quad \text { for all } z \in \mathbb{R}^{m}
$$

Then $f$ is Lipschitz continuous on $\mathbb{R}^{m}$. In particular, if $f$ is $C^{1}$, then there holds

$$
|\nabla f(z)| \leqq C \quad \text { for all } z \in \mathbb{R}^{m}
$$

with a constant $C$ depending only on $m$ and $\Gamma$. and

Lemma 2.8. Suppose that $f: \mathbb{R}^{m} \rightarrow[0, \infty)$ is a convex $C^{1}$-function with $f(0) \leqq \lambda$

$$
f(z) \geqq \gamma|z| \quad \text { for all } z \in \mathbb{R}^{m}
$$

Then there holds

$$
\nabla f(z) \cdot z \geqq \gamma|z|-\lambda \quad \text { for all } z \in \mathbb{R}^{m}
$$

Proof. By the convexity of $f$ we have

$$
\lambda \geqq f(0) \geqq f(z)-\nabla f(z) \cdot z \geqq \gamma|z|-\nabla f(z) \cdot z
$$

for all $z \in \mathbb{R}^{m}$ and the claim follows.

A lemma about generalized minimizers and Dirichlet classes. As discussed above generalized minimizers need not attain prescribed boundary values. The next lemma states that if we move the prescribed boundary values towards the trace of a generalized minimizer, then the minimizing property is preserved. In particular, generalized minimizers are always minimizing with respect to their own boundary values.

Lemma 2.9. For $u_{0}, \tilde{u}_{0} \in W^{1,1}\left(\Omega, \mathbb{R}^{N}\right)$ we consider the Dirichlet classes

$$
\mathscr{D}=u_{0}+W_{0}^{1,1}\left(\Omega, \mathbb{R}^{N}\right) \quad \text { and } \quad \tilde{\mathscr{D}}=\tilde{u}_{0}+W_{0}^{1,1}\left(\Omega, \mathbb{R}^{N}\right)
$$

and we suppose that $f: \mathbb{R}^{N n} \rightarrow[0, \infty)$ is convex with $(\mathrm{H} 1)$. If $u$ is a generalized minimizer of $F$ in $\tilde{\mathscr{D}}$ and

$$
u_{0}(x) \text { is a convex combination of } u(x) \text { and } \tilde{u}_{0}(x) \text { for } \mathscr{H}^{n-1} \text {-a.e. } x \in \partial \Omega,
$$

then $u$ is also a generalized minimizer of $F$ in $\mathscr{D}$. 
Proof. By $(2.8)$ the three vectors $\tilde{u}_{0}-u, \tilde{u}_{0}-u_{0}$, and $u_{0}-u$ point in the same direction, and thus by the 1 -homogeneity of $f^{\infty}$ we have

$$
f^{\infty}\left(\left(\tilde{u}_{0}-u\right) \otimes v_{\Omega}\right)=f^{\infty}\left(\left(\tilde{u}_{0}-u_{0}\right) \otimes v_{\Omega}\right)+f^{\infty}\left(\left(u_{0}-u\right) \otimes v_{\Omega}\right) \quad \mathscr{H}^{n-1} \text {-a.e. on } \partial \Omega
$$

Using this together with the minimality of $u$ in $\tilde{\mathscr{D}}$ we get for any $w \in B V\left(\Omega, \mathbb{R}^{N}\right)$

$$
\begin{gathered}
\mathscr{F}^{\mathscr{D}}[u]=\mathscr{F}^{\tilde{D}}[u]+\int_{\partial \Omega}\left[f^{\infty}\left(\left(u_{0}-u\right) \otimes v_{\Omega}\right)-f^{\infty}\left(\left(\tilde{u}_{0}-u\right) \otimes v_{\Omega}\right)\right] d \mathscr{H}^{n-1} \\
\leqq \mathscr{F}^{\tilde{D}}[w]-\int_{\partial \Omega} f^{\infty}\left(\left(\tilde{u}_{0}-u_{0}\right) \otimes v_{\Omega}\right) d \mathscr{H}^{n-1} \\
=\mathscr{F}^{\mathscr{D}}[w]+\int_{\partial \Omega}\left[f^{\infty}\left(\left(\tilde{u}_{0}-w\right) \otimes v_{\Omega}\right)-f^{\infty}\left(\left(u_{0}-w\right) \otimes v_{\Omega}\right)\right. \\
\left.-f^{\infty}\left(\left(\tilde{u}_{0}-u_{0}\right) \otimes v_{\Omega}\right)\right] d \mathscr{H}^{n-1} .
\end{gathered}
$$

By the convexity of $f^{\infty}$ the integrand in the last integral is nonpositive, and thus $u$ is also minimizing in $\mathscr{D}$.

A comparison principle. Now we come to a comparison principle which incorporates boundary integrals as in (1.8). The principle will only be used in Section 3.3 and we restrict ourselves to a plain version which is sufficient for our needs.

Lemma 2.10. Let $N=1$. Consider $u_{0}, v_{0} \in W^{1,1}(\Omega)$ and assume that $f: \mathbb{R}^{n} \rightarrow[0, \infty)$ is strictly convex. Moreover, suppose that $u \in W^{1,1}(\Omega)$ and $v \in W^{1,1}(\Omega)$ minimize the scalar integrals

$$
\int_{\Omega} f(\nabla w) d x+\int_{\partial \Omega}\left|u_{0}-w\right| d \mathscr{H}^{n-1} \quad \text { and } \quad \int_{\Omega} f(\nabla w) d x+\int_{\partial \Omega}\left|v_{0}-w\right| d \mathscr{H}^{n-1}
$$

respectively, among all $w \in W^{1,1}(\Omega)$, and that $v-u$ is not constant. Then we have the comparison principle

$$
u_{0} \geqq v_{0} \quad \mathscr{H}^{n-1} \text {-a.e. on } \partial \Omega \quad \Rightarrow \quad u \geqq v \quad \mathscr{L}^{n} \text {-a.e. in } \Omega \text {. }
$$

Proof. We first note that $\int_{\Omega} f(\nabla u) d x$ and $\int_{\Omega} f(\nabla v) d x$ are finite as one can see comparing $u$ and $v$ with the zero function. This observation justifies the following computations involving these integrals.

We assume for contradiction that $u_{0} \geqq v_{0}$ holds on $\partial \Omega$ but that

$$
A:=\{x \in \Omega: u(x)<v(x)\}
$$

has positive $\mathscr{L}^{n}$-measure. Then we exploit the minimality of $u$ and $v$ : We compare $u$ with $\tilde{u}:=\frac{u+\max \{u, v\}}{2} \in W^{1,1}(\Omega)$ and $v$ with $\tilde{v}:=\frac{\min \{u, v\}+v}{2} \in W^{1,1}(\Omega)$, and we use the fact that the integrals over $\Omega \backslash A$ cancel out. By this reasoning we find 


$$
\begin{aligned}
& \int_{A} f(\nabla u) d x+\int_{\partial \Omega}\left|u_{0}-u\right| d \mathscr{H}^{n-1} \leqq \int_{A} f\left(\nabla \frac{u+v}{2}\right) d x+\int_{\partial \Omega}\left|u_{0}-\tilde{u}\right| d \mathscr{H}^{n-1}, \\
& \int_{A} f(\nabla v) d x+\int_{\partial \Omega}\left|v_{0}-v\right| d \mathscr{H}^{n-1} \leqq \int_{A} f\left(\nabla \frac{u+v}{2}\right) d x+\int_{\partial \Omega}\left|v_{0}-\tilde{v}\right| d \mathscr{H}^{n-1} .
\end{aligned}
$$

With $A$ also $\{x \in A: \nabla u(x) \neq \nabla v(x)\}$ has positive $\mathscr{L}^{n}$-measure (otherwise $\max \{v-u, 0\}$ would equal a positive constant on $\Omega$, which contradicts our assumption). Taking into account the strict convexity of $f$ we thus have

$$
2 \int_{A} f\left(\nabla \frac{u+v}{2}\right) d x<\int_{A} f(\nabla u) d x+\int_{A} f(\nabla v) d x
$$

Combining the previous three inequalities we come out with an inequality containing only boundary integrals, namely

$$
\int_{\partial \Omega}\left[\left|u_{0}-u\right|+\left|v_{0}-v\right|\right] d \mathscr{H}^{n-1}<\int_{\partial \Omega}\left[\left|u_{0}-\tilde{u}\right|+\left|v_{0}-\tilde{v}\right|\right] d \mathscr{H}^{n-1} .
$$

Now we introduce $\tilde{A}:=\{x \in \partial \Omega: u(x) \leqq v(x)\}$. We recall the definitions ${ }^{11)}$ of $\tilde{u}$ and $\tilde{v}$, and we use $u_{0} \geqq v_{0}$ and $u \leqq v$ on $\tilde{A}$. Distinguishing the three cases $\frac{u+v}{2} \leqq v_{0}, v_{0}<\frac{u+v}{2}<u_{0}$, and $u_{0} \leqq \frac{u+v}{2}$ one verifies

$$
\left|u_{0}-\tilde{u}\right|+\left|v_{0}-\tilde{v}\right|=\left|u_{0}-\frac{u+v}{2}\right|+\left|v_{0}-\frac{u+v}{2}\right| \leqq\left|u_{0}-u\right|+\left|v_{0}-v\right| \quad \text { on } \tilde{A} .
$$

Furthermore, the definitions of $\tilde{u}$ and $\tilde{v}$ also give

$$
\left|u_{0}-\tilde{u}\right|+\left|v_{0}-\tilde{v}\right|=\left|u_{0}-u\right|+\left|v_{0}-v\right| \quad \text { on } \partial \Omega \backslash \tilde{A} .
$$

Since (2.10) and (2.11) are not compatible with (2.9), we have reached a contradiction. Thus, we must have $\mathscr{L}^{n}(A)=0$ and we have established the claim.

Ekeland's variational principle. Finally, we restate the famous variational principle from [30], [31], [32] which plays an important role in the proofs of our uniqueness results.

Lemma 2.11. Suppose that $X$ is a complete metric space, endowed with metric $d$, and that $F: X \rightarrow[0, \infty]$ is a lower semicontinuous functional with $\inf _{X} F<\infty$. If for some $\varepsilon>0$ and some $u \in X$ there holds

then there is a $v \in X$ such that

$$
F[u] \leqq \inf _{X} F+\varepsilon
$$

$$
\begin{gathered}
d(u, v) \leqq \sqrt{\varepsilon} \\
F[v] \leqq F[w]+\sqrt{\varepsilon} d(v, w) \quad \text { for all } w \in X .
\end{gathered}
$$

11) In the proof of Lemma 2.10 - as in the whole paper-we have suppressed an explicit notation for the continuous linear trace operator $T$. However, it should be noted that working with $\tilde{u}$ on $\partial \Omega$ we are implicitly using $T \max \{u, v\}=\max \{T u, T v\}$ on $\partial \Omega$. To establish this equality one first proves $T|u-v|=|T(u-v)|$ (approximating $u-v$ with continuous functions on $\bar{\Omega}$ ) and then writes $2 \max \{u, v\}=u+v+|u-v|$. 
A Young type inequality. The following inequality can be interpreted as a Young inequality in terms of $N$-functions. We state a particular version which is adapted to our needs in Section 5.

Lemma 2.12. For all $t \in[0, \infty)$ and $s \in \mathbb{R}$ there holds

$$
s(1+t) \leqq t \log \left(1+t^{2}\right)+\exp (s)
$$

Proof. Fixing $t$ we have by standard calculus

$$
\begin{aligned}
\max _{s \in \mathbb{R}}[s(1+t)-\exp (s)] & =(1+t) \log (1+t)-(1+t) \\
& \leqq t \log (1+t)-1 \leqq t \log \left(1+t^{2}\right)
\end{aligned}
$$

\section{Various examples}

3.1. Examples of integrands. In this subsection we discuss two classes of integrands.

The first class of examples is the 2-parameter family of integrands given by

$$
f_{\lambda, p}(z):=\left[1+\left(\lambda^{2}+|z|^{2}\right)^{\frac{p}{2}}\right]^{\frac{1}{p}} \quad \text { for } z \in \mathbb{R}^{N n}
$$

where $p \geqq 1$ and $\lambda \geqq 0$ are the parameters. We start recording that the model integrands $e_{\lambda}$ from Section 1.1 are included in this family, precisely the relation is given by

$$
f_{\lambda, 1}=1+e_{\lambda} \text { and } f_{\lambda, 2}=e \sqrt{1+\lambda^{2}}
$$

It is easily checked that $f_{\lambda, p}$ is $C^{2}$ (in the case $\lambda=0,1<p<2$ only away from 0 ) and convex with (H1). Moreover, it is rotationally symmetric and thus satisfies all the relevant structure conditions discussed in Section 1.2, that is the conditions (1.10), (1.12), and in particular $(\mathrm{H} 3)$. Additionally, we have $\left(f_{\lambda, p}\right)^{\infty}(z)=|z|$ and thus (H4) is also available (compare Remark 1.15).

Hence, it remains to discuss the assumption (H2) which is the limit case $\mu=3$ of the $\mu$-ellipticity condition $\left(\mathrm{H} 2_{\mu}\right)$. In fact, it can be checked by elementary computations that $f_{\lambda, p}$ satisfies $\left(\mathrm{H} 2_{\mu}\right)$ with

$$
\mu= \begin{cases}p+1 & \text { if } p>1, \lambda>0 \text { or } p=2 \\ 3 & \text { if } p=1, \lambda>0\end{cases}
$$

The main results of Section 1.2 are valid for $\mu \leqq 3$ and thus they cover $f_{\lambda, p}$ for $1 \leqq p \leqq 2$, $\lambda>0$ and for $p=2, \lambda=0$. However, for $\mu<3$ the results of Theorem 1.10 (and actually even $C^{1, \alpha}$-regularity) were already known from [15] and thus the most interesting feature of the present paper is the inclusion of the limit case $\mu=3$ and the integrands $e_{\lambda}$ from Section 1.1. 
We believe that the remaining cases $\lambda=0, p \neq 2$ are of some interest though the respective integrands

$$
f_{0, p}(z)=\left(1+|z|^{p}\right)^{\frac{1}{p}}
$$

do not fulfil the condition $\left(\mathrm{H} 2_{\mu}\right)$ for any value of $\mu$, essentially due to the degenerate resp. singular behavior of $f_{0, p}$ at the origin. For this reason most results in this paper-with the exception of Theorem 1.11 - do not apply to these integrands, which we plan to investigate in the forthcoming paper [12].

Even though the integrands $f_{\lambda, p}$ (and in particular $e_{\lambda}$ ) provide the main motivation for the present paper, this family of examples is quite limited. In particular, as remarked above all the integrands $f_{\lambda, p}$ have the same recession function. We take this as a motivation to provide a second class of examples. Basically, we will prescribe an arbitrary 1-homogeneous and convex function $g$ and construct a suitable $\mu$-elliptic integrand $f$ with $f^{\infty}=g$. We believe that this construction might be of some independent interest but in particular we have the following two applications in mind. On the one hand we deduce that the convexity assumption $(\mathrm{H} 4)$ for $f^{\infty}$ is independent from $(\mathrm{H} 1)$ and $\left(\mathrm{H} 2_{\mu}\right)$ and cannot be concluded from the strict convexity of $f$ (Remark 3.3). On the other hand the construction will be useful for the example in Section 3.4 below.

Next let us supply the precise ${ }^{12)}$ statements.

Proposition 3.1. Suppose that $g$ is a norm on $\mathbb{R}^{m}$ and $\mu>1$ is given. Then there exists a smooth convex function $f: \mathbb{R}^{m} \rightarrow[0, \infty)$ with $(\mathrm{H} 1), \nabla f(0)=0$,

$$
f^{\infty}=g
$$

and such that the left-hand inequality in $\left(\mathrm{H}_{2}\right)$ holds. In the case $\mu>2$ we may additionally achieve $f \geqq g$. Moreover, if $g$ has one (or both) of the following additional properties, then $f$ may be chosen such that it has the corresponding property (or properties):

- $g$ is even in one of its variables $\rightsquigarrow f$ is even in the same variable.

- $g$ is locally $C^{1,1}$ on $\mathbb{R}^{m} \backslash\{0\} \rightsquigarrow f$ satisfies the right-hand inequality in $\left(\mathrm{H} 2_{\mu}\right)$.

Remark 3.2. The requirement $\mu>2$ in Proposition 3.1 will have consequences in Sections 3.3 and 3.4 (see in particular the proof of Lemma 3.13 and Remark 3.15) and is in fact optimal in the following sense: There exists no $C^{2}$-function $f$ on $\mathbb{R}^{m}$ with $f \geqq f^{\infty}$ such that the left-hand inequality in $(\mathrm{H} 2 \mu)$ holds with $\mu \leqq 2$.

The last claim can be proved elementarily. Nevertheless, we briefly sketch the argument since - in our opinion - it is not completely straightforward:

12) Some of the following statements will be provided for an arbitrary Euclidean space $\mathbb{R}^{m}$ instead of $\mathbb{R}^{N n}$. When we write (H1) or (H2) in this context we refer-of course-to the respective conditions for $z, \tilde{z} \in \mathbb{R}^{m}$ instead of $z, \tilde{z} \in \mathbb{R}^{N n}$. 
Proof of the non-existence claim in Remark 3.2. It suffices to deal with the case $\mu=2, m=1$ (restricting $f$ to lines through the origin). We consider the auxiliary function

$$
h(t):=t \arctan t-\frac{1}{2} \log \left(1+t^{2}\right)
$$

the reason for this choice being $h^{\prime \prime}(t)=1 /\left(1+t^{2}\right)$. If $f$ satisfies the left-hand inequality in $\left(\mathrm{H} 2_{\mu}\right)$ with $\mu=2$, then $f^{\prime \prime} \geqq \gamma h^{\prime \prime}$ holds for some $\gamma>0$, and $f-\gamma h$ is convex. In particular, for $s, t \in \mathbb{R}$ it follows that

$$
(f-\gamma h)(s)+(f-\gamma h)^{\prime}(s)(t-s) \leqq(f-\gamma h)(t) .
$$

Dividing by $t$ and passing $t \rightarrow \infty$ we come out with

$$
f^{\prime}(s) \leqq f^{\infty}(1)+\gamma h^{\prime}(s)-\gamma \frac{\pi}{2}
$$

Integrating the last inequality with respect to $s$ yields

$$
\begin{aligned}
f(s) & \leqq f(0)+s f^{\infty}(1)+\gamma h(s)-\gamma \frac{\pi}{2} s \\
& \leqq f(0)+f^{\infty}(s)-\frac{\gamma}{2} \log \left(1+s^{2}\right)
\end{aligned}
$$

for all $s \geqq 0$. Choosing $s$ sufficiently large we see that $f \geqq f^{\infty}$ does not hold.

Remark 3.3. Proposition 3.1 can be used to construct a smooth integrand $f$ such that $(\mathrm{H} 1)$ and $\left(\mathrm{H} 2_{\mu}\right)$ hold, but $(\mathrm{H} 4)$ still fails. To this end the proposition is applied with a suitable non-strictly convex norm such as $g(z)=\sum_{\alpha=1}^{N}\left|z^{\alpha}\right|$ for $m=N n$ and $N>1$.

However, a structure assumption on $g$ yields (H3) and (H4):

Proposition 3.4. In the case $m=N n$ the list of additional properties in Proposition 3.1 may be extended by the following two points:

- $g$ has the structure in (1.10) $\rightsquigarrow f$ satisfies (1.10) (and thus (H3) and (H4)).

- $g$ has the structure in (1.12) $\rightsquigarrow f$ satisfies (1.12).

Then the construction of Proposition 3.1 is possible in such a way that any combination of properties of $g$ from this extended list leads to the corresponding properties of $f$.

The proof of Proposition 3.1 and Proposition 3.4 is based on the following lemma. Indeed, the assertion in the lemma is quite plausible, but we include its proof for the sake of completeness. 
Lemma 3.5. Suppose that $g$ is a norm on $\mathbb{R}^{m}$. Then there exists a smooth convex function $f_{*}: \mathbb{R}^{m} \rightarrow[0, \infty)$ with $\left(f_{*}\right)^{\infty}=g$ and $f_{*} \geqq g$ such that $\nabla f_{*}(0)=0$ holds and $\nabla^{2} f_{*}(0)$ is positive. Moreover, we may achieve additional properties as indicated in the following:

- $g$ is even in one of its variables $\rightsquigarrow f_{*}$ is even in the same variable.

- $g$ is locally $C^{1,1}$ on $\mathbb{R}^{m} \backslash\{0\} \rightsquigarrow f_{*}$ satisfies the right-hand inequality in $\left(\mathrm{H} 2{ }_{\mu}\right)$.

Proof. We start by introducing the positive numbers

$$
a:=\min _{|x|=1} g(x) \quad \text { and } \quad A:=\max _{|x|=1} g(x),
$$

and we write $\mathrm{Co} h$ for the convex envelope of the auxiliary function $h: \mathbb{R}^{m} \rightarrow(0, \infty)$ which is given by

$$
h(x):= \begin{cases}g(x) & \text { if }|x| \geqq 1 \\ \frac{a}{2}+\frac{A^{2}}{2 a}|x|^{2} & \text { if }|x|<1\end{cases}
$$

Now we prove that Co $h$ equals $h$ outside the unit ball. We first observe

$$
g(x) \leqq A|x| \leqq \frac{a}{2}+\frac{A^{2}}{2 a}|x|^{2}
$$

Thus we have $g \leqq h$ on $\mathbb{R}^{m}$ and by the properties of the convex envelope we deduce $g \leqq \mathrm{Co} h \leqq h$ on $\mathbb{R}^{m}$ which gives the equality $\mathrm{Co} h=g$ outside the unit ball $B_{1}$. Next we prove that $\mathrm{Co} h$ is a paraboloid near 0 . To this end we consider $\varepsilon \in(0,1 / 2)$ and $p_{\varepsilon}: \mathbb{R}^{m} \rightarrow(0, \infty)$ defined as

$$
p_{\varepsilon}(x):= \begin{cases}\frac{a}{2}+\frac{2 A^{2} \varepsilon^{2}}{a}+\frac{2 A^{2} \varepsilon}{a}(|x|-2 \varepsilon) & \text { if }|x| \geqq 2 \varepsilon, \\ \frac{a}{2}+\frac{A^{2}}{2 a}|x|^{2} & \text { if }|x| \leqq 2 \varepsilon .\end{cases}
$$

We note that $p_{\varepsilon}$ is constructed from the convex, increasing function $t \mapsto \frac{a}{2}+\frac{A^{2}}{2 a} t^{2}$ on the half-line $[0, \infty)$, linearizing it for $t \geqq 2 \varepsilon$ and then rotating it. Thus, $p_{\varepsilon}$ is convex, $p_{\varepsilon}(x) \leqq h(x)$ for $|x|<1$, and $p_{\varepsilon}(x)=h(x)$ for $|x| \leqq 2 \varepsilon$. Let us fix $\varepsilon$ (depending only on $a$ and $A$ ) small enough that

$$
\frac{a}{2}+\frac{2 A^{2} \varepsilon^{2}}{a}+\frac{2 A^{2} \varepsilon}{a}(1-2 \varepsilon) \leqq a \text { and } \quad \frac{2 A^{2} \varepsilon}{a} \leqq a
$$

hold. Now (by the first smallness condition) we have $p_{\varepsilon}(x) \leqq a \leqq g(x)$ for $|x|=1$ and then (by the second one)

$$
p_{\varepsilon}(x)=p_{\varepsilon}(x /|x|)+\frac{2 A^{2} \varepsilon}{a}(|x|-1) \leqq g(x /|x|)+a(|x|-1) \leqq g(x)=h(x)
$$


for $|x| \geqq 1$. All in all we find $p_{\varepsilon} \leqq h$ on $\mathbb{R}^{m}$ and using the properties of the convex envelope again we infer $p_{\varepsilon} \leqq \operatorname{Co} h \leqq h$ on $\mathbb{R}^{m}$. Recalling $p_{\varepsilon}(x)=h(x)$ for $|x| \leqq 2 \varepsilon$ we deduce that Co $h$ and $p_{\varepsilon}$ coincide on $B_{2 \varepsilon}$.

Finally, we define $f_{*}$ as the mollification of $\mathrm{Co} h$ with smoothing radius $\varepsilon$, that is,

$$
f_{*}(x):=\int_{\mathbb{R}^{m}} \operatorname{Coh}(x-\varepsilon y) \eta(y) d y=\int_{\mathbb{R}^{m}} \eta_{\varepsilon}(x-y) \operatorname{Coh}(y) d y,
$$

where $\eta$ is a (usual) smooth, symmetric mollifying kernel, compactly supported in $B_{1}$, and $\eta_{\varepsilon}(x):=\varepsilon^{-m} \eta(x / \varepsilon)$. Since convexity is preserved under mollification, $f_{*}$ is convex. Moreover, by Jensen's inequality there holds

$$
f_{*}(x) \geqq \operatorname{Coh}\left(\int_{\mathbb{R}^{m}}(x-\varepsilon y) \eta(y) d y\right)=\operatorname{Coh}(x) \geqq g(x)
$$

and for $x \neq 0$ and $s \gg 1$ we have

$$
\frac{f_{*}(s x)}{s}=\frac{1}{s_{B_{1}}} \int_{B_{1}} g(s x-\varepsilon y) \eta(y) d y=\int_{B_{1}} g(x-\varepsilon y / s) \eta(y) d y \underset{s \rightarrow \infty}{\longrightarrow} g(x),
$$

which means $\left(f_{*}\right)^{\infty}=g$. Next, computing the mollification of Co $h=p_{\varepsilon}$ near 0 explicitly we get $\nabla f_{*}(0)=0$ and $\nabla^{2} f_{*}(0)=\frac{A^{2}}{a} I_{m}$, where $I_{m}$ denotes the $(m \times m)$-unit matrix.

The claim about even dependence on the variables is obvious.

Finally, if $g$ is locally $C^{1,1}$, then by 1-homogeneity there holds ${ }^{13)}\left|\nabla^{2} g(z)\right| \leqq C|z|^{-1}$. Now, since $\nabla^{2} f_{*}$ is smooth and coincides near infinity with the mollification of $\nabla^{2} g$, we deduce

$$
\nabla^{2} f_{*}(z) \leqq C(1+|z|)^{-1}
$$

as claimed.

Proof of Proposition 3.1. Denote by $f_{*}$ the function constructed in Lemma 3.5. Now we let $f_{k}(z):=k\left[f_{*}(z / k)-f_{*}(0)\right]$ for all $k \in \mathbb{N}$ and evidently $f_{k}$ is convex with $f_{k}(0)=0$, $\nabla f_{k}(0)=0$, and $\left(f_{k}\right)^{\infty}=g$. By $(2.5)$ we thus have $0 \leqq f_{k} \leqq g$ on $\mathbb{R}^{m}$. Next we set $\zeta(\mu):=\sum_{k=1}^{\infty} k^{-\mu}$ and

$$
f:=M+\frac{1}{\zeta(\mu)} \sum_{k=1}^{\infty} k^{-\mu} f_{k}
$$

where $M \geqq 0$ will be chosen below. Then $f$ is convex with $f(0)=M, \nabla f(0)=0$. We note that on the one hand there hold $M \leqq f \leqq M+g$ and thus $f^{\infty} \leqq g$ on $\mathbb{R}^{m}$, and on the other

13) By Rademacher's theorem $\nabla^{2} g(z)$ exists for $\mathscr{L}^{m}$-a.e. $z \in \mathbb{R}^{m}$. 
hand $f^{\infty} \geqq \frac{1}{\zeta(\mu)} \sum_{k=1}^{K} k^{-\mu}\left(f_{k}\right)^{\infty}=\frac{1}{\zeta(\mu)} \sum_{k=1}^{K} k^{-\mu} g$ is valid on $\mathbb{R}^{m}$ for every $K \in \mathbb{N}$. In conclusion we thus find $f^{\infty}=g$ on $\mathbb{R}^{m}$ as claimed. Moreover, computing the $l$ th derivative of the series in (3.1) term by term, we end up with another series which can locally be majorized by $\sum_{k=1}^{\infty} k^{1-l-\mu}$ and thus converges locally uniformly for every $l \in \mathbb{N}$. Consequently, the limit function $f$ has a continuous $l$ th derivative for every $l \in \mathbb{N}$ and is smooth on $\mathbb{R}^{m}$. Next, let us derive the left-hand inequality in $\left(\mathrm{H} 2_{\mu}\right)$. We first observe that there exists an $\varepsilon>0$ such that

$$
\nabla^{2} f_{*}(z)(\tilde{z}, \tilde{z}) \geqq \varepsilon|\tilde{z}|^{2} \quad \text { for all } z \text { with }|z|<\varepsilon \text {. }
$$

Then for an arbitrary $z$ we choose $k_{0} \in \mathbb{N}$ such that $\left(k_{0}-1\right) \varepsilon \leqq|z|<k_{0} \varepsilon$ and find

$$
\begin{aligned}
\nabla^{2} f(z)(\tilde{z}, \tilde{z}) & \geqq \frac{1}{\zeta(\mu)} \sum_{k=1}^{\infty} k^{-\mu} \nabla^{2} f_{k}(z)(\tilde{z}, \tilde{z}) \geqq \frac{1}{\zeta(\mu)} \sum_{k=k_{0}}^{\infty} k^{-1-\mu} \nabla^{2} f_{*}(z / k)(\tilde{z}, \tilde{z}) \\
& \geqq \frac{\varepsilon|\tilde{z}|^{2}}{\zeta(\mu)} \sum_{k=k_{0}}^{\infty} k^{-1-\mu} \geqq \frac{\varepsilon|\tilde{z}|^{2}}{\zeta(\mu)} \int_{k_{0}}^{\infty} t^{-1-\mu} d t=\frac{\varepsilon|\tilde{z}|^{2}}{\zeta(\mu) \mu} k_{0}^{-\mu} \\
& \geqq c(\varepsilon, \mu)(1+|z|)^{-\mu}|\tilde{z}|^{2}
\end{aligned}
$$

Thus, we have established the left-hand side of $\left(\mathrm{H} 2_{\mu}\right)$. In order to derive $(\mathrm{H} 1)$ we recall $f(0)=M \geqq 0$ and $\nabla f(0)=0$, and we moreover note that $\nabla^{2} f(\xi)(z, z) \geqq \frac{1}{\zeta(\mu)} \nabla^{2} f_{*}(\xi)(z, z)$ holds by (3.1) and the convexity of $f_{k}$. From these properties and (3.2) we get

$$
\begin{aligned}
f(z) & \geqq \frac{1}{\zeta(\mu)} \int_{0}^{1} \int_{0}^{1} \nabla^{2} f_{*}(s t z) d s t d t(z, z) \\
& \geqq \frac{\varepsilon}{\zeta(\mu)} \int_{0}^{1} \int_{0}^{\frac{\varepsilon}{|z|}} d s t d t|z|^{2} \geqq \frac{\varepsilon^{2}}{2 \zeta(\mu)}|z| \quad \text { for }|z| \geqq \varepsilon .
\end{aligned}
$$

Thus, choosing $M \geqq \frac{\varepsilon^{3}}{2 \zeta(\mu)}$ we have $f(z) \geqq \frac{\varepsilon^{2}}{2 \zeta(\mu)}|z|$ for all $z \in \mathbb{R}^{m}$ and the left-hand side of (H1) holds. Moreover, the right-hand side of (H1) follows from $f \leqq M+g$ and the 1-homogeneity of $g$. Finally, we exploit the inequality $f_{*} \geqq g$ from Lemma 3.5. Recalling the above definitions of $f_{k}, f$, and $\zeta(\mu)$ and invoking the 1-homogeneity of $g$, we thus get

$$
f(z) \geqq M+\frac{1}{\zeta(\mu)} \sum_{k=1}^{\infty} k^{-\mu} k\left[g(z / k)-f_{*}(0)\right]=M+g(z)-\frac{f_{*}(0)}{\zeta(\mu)} \sum_{k=1}^{\infty} k^{1-\mu}
$$

In the case $\mu>2$ the last sum converges and thus enlarging $M$ if necessary we arrive at $f \geqq g$.

The even dependence on certain variables is preserved through the construction. 
Finally, if $g$ is locally $C^{1,1}$ on $\mathbb{R}^{m} \backslash\{0\}$, then Lemma 3.5 gives $\left|\nabla^{2} f_{*}(z)\right| \leqq C(1+|z|)^{-1}$. Tracing the consequences of this bound we infer first

$$
\left|\nabla^{2} f_{k}(z)\right| \leqq C(k+|z|)^{-1} \leqq C(1+|z|)^{-1}
$$

and then

$$
\left|\nabla^{2} f(z)\right| \leqq C(1+|z|)^{-1}
$$

with the same constant $C$ in all these conditions.

Lemma 3.6. Suppose that a function $g$ on $\mathbb{R}^{\text {Nn }}$ has both structures, the one from (1.10) and the one from (1.12). Then $g$ is rotationally symmetric, i.e., $g(z)$ depends only on $|z|$.

Proof. By assumption we have

$$
g(z)=\tilde{g}\left(\left|z_{1}\right|,\left|z_{2}\right|, \ldots,\left|z_{n}\right|\right)=\tilde{\tilde{g}}\left(\left|z^{1}\right|,\left|z^{2}\right|, \ldots,\left|z^{N}\right|\right) .
$$

Plugging a matrix $z \in \mathbb{R}^{N n}$ with $z^{1}=x \in[0, \infty)^{n}$ and $z^{2}=z^{3}=\cdots=z^{N}=0$ into (3.3) we deduce $\tilde{g}\left(x_{1}, x_{2}, \ldots, x_{n}\right)=\tilde{\tilde{g}}(|x|, 0, \ldots, 0)$. We hence get

$$
g(z)=\tilde{g}\left(\left|z_{1}\right|,\left|z_{2}\right|, \ldots,\left|z_{n}\right|\right)=\tilde{\tilde{g}}(|z|, 0, \ldots, 0) \quad \text { for all } z \in \mathbb{R}^{N n} .
$$

Proof of Proposition 3.4. We will deal with the case that $g$ satisfies (1.10) omitting the treatment of (1.12), which is completely analogous. Moreover, the case that $g$ satisfies both conditions, (1.10) and (1.12), will not be discussed in detail since exploiting Lemma 3.6 it can be treated by simplified versions of the following arguments.

We introduce the abbreviation $S z$ for $\left(\left|z_{1}\right|, \ldots,\left|z_{n}\right|\right.$ ) (note $\left.|S z|=|z|\right)$ and write down (1.10) for $g$, that is,

$$
g(z)=\tilde{g}(S z)
$$

for some function $\tilde{g}$, first defined on $[0, \infty)^{n}$. After extending $\tilde{g}$ to all of $\mathbb{R}^{n}$ as an even function in each of its $n$ variables we apply Proposition 3.1 (with $m=n$ ) to $\tilde{g}$. We come out with a smooth convex function $\tilde{f}: \mathbb{R}^{n} \rightarrow[0, \infty)$, even in all of its variables, satisfying $\nabla \tilde{f}(0)=0$ and $\tilde{f}^{\infty}=\tilde{g}$, and such that $(\mathrm{H} 1)$ and $\left(\mathrm{H} 2{ }_{\mu}\right)$ hold for $\tilde{f}$. We define $f$ by letting

$$
f(z):=\tilde{f}(S z) .
$$

Obviously $f$ has the structure (1.10), but since we have modified the above construction we still need to check that all the properties in Proposition 3.1 carry over from $\tilde{f}$ to $f$. It is easy to see from the corresponding properties of $\tilde{f}$ that $f$ is convex and satisfies $\nabla f(0)=0$, $f^{\infty}=g$, and (H1). Moreover, in the case $\mu>2$ by Proposition 3.1 we may achieve $\tilde{f} \geqq \tilde{g}$, and obviously this gives $f \geqq g$. In order to deal with the derivatives of $f$ let us make two observations: Primarily, since $\tilde{f}$ is even, $\partial^{i} \tilde{f}(S z)$ vanishes for $z_{i}=0$; secondly, as a consequence, for $j \neq i$ also $\partial^{i} \partial^{j} \tilde{f}(S z)=\partial^{j} \partial^{i} \tilde{f}(S z)$ vanishes for $z_{i}=0$. Starting from the fact that $\tilde{f}$ is smooth and even in all variables, one can then check that $f$ has continuous derivatives of any order, even near those points $z$ with $z_{i}=0$. (In particular for the second 
derivatives this can be seen from (3.5) keeping the preceding observations in mind.) Hence, $f$ is smooth. Next we will verify $\left(\mathrm{H} 2_{\mu}\right)$ for $f$. By $\left(\mathrm{H} 2_{\mu}\right)$ for $\tilde{f}$ we have

$$
\partial^{i} \tilde{f}(S z)=\int_{0}^{\left|z_{i}\right|} \partial^{i} \partial^{i} \tilde{f}\left(\left|z_{1}\right|, \ldots,\left|z_{i-1}\right|, t,\left|z_{i+1}\right|, \ldots,\left|z_{n}\right|\right) d t \geqq c(1+|z|)^{-\mu}\left|z_{i}\right|
$$

for all $z \in \mathbb{R}^{N n}$. Now let us compute the second derivatives of $f$ in terms of $\tilde{f}$. We find

$$
\frac{\partial^{2} f}{\partial z_{i}^{\alpha} \partial z_{j}^{\beta}}(z)=\left[\partial^{i} \partial^{j} \tilde{f}(S z)-\frac{\delta_{i j}}{\left|z_{i}\right|} \partial^{i} \tilde{f}(S z)\right] \frac{z_{i}^{\alpha} z_{j}^{\beta}}{\left|z_{i}\right|\left|z_{j}\right|}+\frac{\delta_{i j} \delta^{\alpha \beta}}{\left|z_{i}\right|} \partial^{i} \tilde{f}(S z) .
$$

Using $\left(\mathrm{H} 2{ }_{\mu}\right)$ for $\tilde{f}$ and $(3.4)$ we see

$$
\begin{aligned}
\nabla^{2} f(z)(\tilde{z}, \tilde{z}) & =\sum_{i, j=1}^{n} \partial^{i} \partial^{j} \tilde{f}(S z) \frac{z_{i} \cdot \tilde{z}_{i}}{\left|z_{i}\right|} \frac{z_{j} \cdot \tilde{z}_{j}}{\left|z_{j}\right|}+\sum_{i=1}^{n} \partial^{i} \tilde{f}(S z) \frac{\left|z_{i}\right|^{2}\left|\tilde{z}_{i}\right|^{2}-\left(z_{i} \cdot \tilde{z}_{i}\right)^{2}}{\left|z_{i}\right|^{3}} \\
& \geqq c(1+|z|)^{-\mu} \sum_{i=1}^{n} \frac{\left(z_{i} \cdot \tilde{z}_{i}\right)^{2}}{\left|z_{i}\right|^{2}}+c(1+|z|)^{-\mu} \sum_{i=1}^{n} \frac{\left|z_{i}\right|^{2}\left|\tilde{z}_{i}\right|^{2}-\left(z_{i} \cdot \tilde{z}_{i}\right)^{2}}{\left|z_{i}\right|^{2}} \\
& =c(1+|z|)^{-\mu}|\tilde{z}|^{2}
\end{aligned}
$$

and we have verified the left-hand inequality in $\left(\mathrm{H} 2_{\mu}\right)$ for $f$.

Finally, we discuss the interaction of (1.10) with the additional properties of Proposition 3.1. In fact, the claims about even dependence on the variables are trivial, and imposing the $C^{1,1}$-assumption on $g$ we argue as follows to derive the corresponding property of $f$. Under the $C^{1,1}$-assumption Proposition 3.1 gives the right-hand side of $(\mathrm{H} 2 \mu)$ for $\tilde{f}$. Integrating as in (3.4) we find the upper bound

$$
\partial^{i} \tilde{f}(S z) \leqq C \frac{\left|z_{i}\right|}{1+\left|\left(z_{1}, \ldots, z_{i-1}, z_{i+1}, \ldots, z_{n}\right)\right|} .
$$

We distinguish the cases of $\left|z_{i}\right|$ greater and not greater than $1+\left|\left(z_{1}, \ldots, z_{i-1}, z_{i+1}, \ldots, z_{n}\right)\right|$. Using Lemma 2.7 in the first situation and (3.6) in the second one we come out, for all $z \in \mathbb{R}^{N n}$, with the inequality

$$
\partial^{i} \tilde{f}(S z) \leqq C \frac{\left|z_{i}\right|}{1+|z|}
$$

Now we use $\left(\mathrm{H} 2_{\mu}\right)$ for $\tilde{f}$ and (3.7) on the right-hand side of (3.5). Keeping in mind that $\partial^{i} \tilde{f}(S z) \geqq 0$ we get

$$
\begin{aligned}
\nabla^{2} f(z)(\tilde{z}, \tilde{z}) & \leqq \sum_{i, j=1}^{n} \partial^{i} \partial^{j} \tilde{f}(S z) \frac{z_{i} \cdot \tilde{z}_{i}}{\left|z_{i}\right|} \frac{z_{j} \cdot \tilde{z}_{j}}{\left|z_{j}\right|}+\sum_{i=1}^{n} \frac{\partial^{i} \tilde{f}(S z)}{\left|z_{i}\right|}|\tilde{z}|^{2} \\
& \leqq C(1+|z|)^{-1}|\tilde{z}|^{2}
\end{aligned}
$$

Thus, the left-hand inequality in $\left(\mathrm{H} 2_{\mu}\right)$ is valid for $f$ as claimed. 
3.2. Proof of Theorem 1.5. In this subsection we work for $n=2$ on the twodimensional annulus

$$
B_{2} \backslash B_{1}=\left\{x \in \mathbb{R}^{2}: 1<|x|<2\right\}
$$

from Theorem 1.5.

The proof of Theorem 1.5 is motivated by a classical example from the theory of area minimizing graphs; see [46], Example 12.15. In that example one prescribes rotationally symmetric boundary values on $\partial\left(B_{2} \backslash B_{1}\right)$ in the case $N=1$. The symmetry allows to reduce to a 1-dimensional variational problem, whose Euler equation can be explicitly computed and solved. For a suitable choice of a parameter it can be seen that the unique generalized minimizer does not attain the boundary values.

As explained in the introduction we are interested in a similar vectorial example exhibiting a more complicated jump at the boundary. For our purposes it is compulsory that the boundary values are not contained in a 1-dimensional affine subspace and thus we may not choose them rotationally symmetric. Instead we use the function $u_{0}$ from Theorem 1.5 exhibiting a different kind of symmetry. However, we can still reduce to a scalar, 1-dimensional problem, but the Euler equation of the reduced problem is quite complicated and there is few hope to find explicit formulas for the solutions. Thus, in the following we will provide a somewhat different line of argument.

For the remainder of this subsection let us fix $N=2$ and the boundary values $u_{0}$ from Theorem 1.5, that is,

$$
u_{0}(x)= \begin{cases}M x & \text { for } x \in \partial B_{1} \\ 0 & \text { for } x \in \partial B_{2}\end{cases}
$$

where $M \in \mathbb{R}$ is a constant.

Lemma 3.7. Let $n=N=2$ and $\Omega=B_{2} \backslash B_{1}$. There exists a unique generalized minimizer $u \in W^{1,1}\left(\Omega, \mathbb{R}^{2}\right)$ of $E_{1}$ in $\mathscr{D}=u_{0}+W_{0}^{1,1}\left(\Omega, \mathbb{R}^{2}\right)$ and it can be written as

$$
u(x)=v(|x|) \frac{x}{|x|} \quad \text { for } x \in \Omega .
$$

Here, $v$ is the unique minimizer in $W^{1,1}(1,2)$ of the 1-dimensional scalar integral $H$, defined for $w \in W^{1,1}(1,2)$ by

$$
H[w]:=\int_{1}^{2} t \sqrt{1+w^{\prime}(t)^{2}+t^{-2} w(t)^{2}} d t+|w(1)-M|+2|w(2)| .
$$

Proof. By Theorem 1.2 and Theorem 1.3 (or alternatively by Corollary 1.13) the set $\mathscr{M}_{1}^{\mathscr{D}}$ of generalized minimizers of $E_{1}$ in $\mathscr{D}$ satisfies

$$
\mathscr{M}_{1}^{\mathscr{D}}=\{\bar{u}+t \bar{y}: t \in[-1,1]\} \subset W^{1,1}\left(\Omega, \mathbb{R}^{2}\right)
$$

for some particular minimizer $\bar{u} \in W^{1,1}\left(\Omega, \mathbb{R}^{2}\right)$ and some $\bar{y} \in \mathbb{R}^{2}$. For $u \in \mathscr{M}_{1}^{\mathscr{D}}$ and an orthogonal transformation $T \in \mathbb{O}(2)$ we define a function $u_{T} \in W^{1,1}\left(\Omega, \mathbb{R}^{2}\right)$ by

$$
u_{T}(x):=T u\left(T^{-1} x\right) \text { for } x \in \Omega .
$$


Then noting

$$
\nabla u_{T}(x)=T \nabla u\left(T^{-1} x\right) T^{-1}, \quad\left|\nabla u_{T}(x)\right|=\left|\nabla u\left(T^{-1} x\right)\right|, \quad T u_{0}\left(T^{-1} x\right)=u_{0}(x)
$$

we find

$$
\mathscr{E}_{1}^{\mathscr{D}}\left[u_{T}\right]=\mathscr{E}_{1}^{\mathscr{D}}[u]
$$

and thus $u_{T} \in \mathscr{M}_{1}^{\mathscr{D}}$. Applying this observation to $\bar{u}$ and $\bar{u}+\bar{y}$ we have $\bar{u}_{T} \in \mathscr{M}_{1}^{\mathscr{D}}$ and $\bar{u}_{T}+T \bar{y}=(\bar{u}+\bar{y})_{T} \in \mathscr{M}_{1}^{\mathscr{D}}$. By the above representation of $\mathscr{M}_{1}^{\mathscr{D}}$ this means that for every $T \in \mathbb{O}(2)$ there exists a $t \in \mathbb{R}$ with $T \bar{y}=t \bar{y}$. However, this can only happen for $\bar{y}=0$, and thus $\mathscr{M}_{1}^{\mathscr{D}}$ contains just one unique minimizer $u$. In conclusion for all $T \in \mathbb{O}(2)$ we have

$$
u(x)=T u\left(T^{-1} x\right) \quad \text { for } \mathscr{L}^{2} \text {-a.e. } x \in \Omega
$$

Using $T=\frac{1}{|x|}\left(\begin{array}{cc}x_{1} & -x_{2} \\ x_{2} & x_{1}\end{array}\right)$ in (3.9) we deduce

$$
u(x)=v(|x|) \frac{x}{|x|}+\tilde{v}(|x|) \frac{\left(-x_{2}, x_{1}\right)}{|x|} \quad \text { for } \mathscr{L}^{2} \text {-a.e. } x \in \Omega
$$

where we abbreviated the component functions on the $x_{1}$-axis as follows:

$$
v(t):=u_{1}(t, 0) \quad \text { and } \quad \tilde{v}(t):=u_{2}(t, 0)
$$

Here, it should be noted that as another consequence of (3.9) these formulas define functions $v$ and $\tilde{v}$ in $W^{1,1}(1,2)$. Next we compute the quantities in $\mathscr{E}_{1}^{\mathscr{D}}[u]$ :

$$
\begin{array}{ll}
|\nabla u(x)|^{2}=v^{\prime}(|x|)^{2}+\tilde{v}^{\prime}(|x|)^{2}+\left(\frac{v(|x|)}{|x|}\right)^{2}+\left(\frac{\tilde{v}(|x|)}{|x|}\right)^{2} & \text { for } \mathscr{L}^{2} \text {-a.e. } x \in \Omega, \\
\left|u(x)-u_{0}(x)\right|^{2}=|v(|x|)-M|^{2}+\tilde{v}(|x|)^{2} & \text { for } \mathscr{H}^{1} \text {-a.e. } x \in \partial B_{1}, \\
\left|u(x)-u_{0}(x)\right|^{2}=v(|x|)^{2}+\tilde{v}(|x|)^{2} & \text { for } \mathscr{H}^{1} \text {-a.e. } x \in \partial B_{2} .
\end{array}
$$

Let us consider $\hat{v}(x):=v(|x|) x /|x|$. Replacing $u$ with $\hat{v}$ on the left-hand sides of (3.10) corresponds to replacing $\tilde{v}$ with 0 on the right-hand sides. Thus we have $\mathscr{E}_{1}^{\mathscr{D}}[\hat{v}] \leqq \mathscr{E}_{1}^{\mathscr{D}}[u]$, and by the above uniqueness of the minimizer $u$ we deduce $\hat{v}=u$; in other words $\tilde{v}$ vanishes and we have verified the representation claimed in the lemma.

It remains to establish the minimizing property of $v$. To this end we apply (3.10) (with $\tilde{v}=0$ ) and get by radial integration

$$
\begin{aligned}
\mathscr{E}_{1}^{\mathscr{D}}[u]= & \int_{B_{2} \backslash B_{1}} \sqrt{1+v^{\prime}(|x|)^{2}+|x|^{-2} v(|x|)^{2}} d x+\int_{\partial B_{1}}|v(|x|)-M| d \mathscr{H}^{1}(x) \\
& +\int_{\partial B_{2}}|v(|x|)| d \mathscr{H}^{1}(x)=2 \pi H[v] .
\end{aligned}
$$


Writing $\hat{w}(x):=w(|x|) x /|x|$ for an arbitrary $w \in W^{1,1}(1,2)$ an analogous computation gives $\mathscr{E}_{1}^{\mathscr{D}}[\hat{w}]=2 \pi H[w]$ and thus

$$
2 \pi H[v]=\mathscr{E}_{1}^{\mathscr{D}}[u] \leqq \mathscr{E}_{1}^{\mathscr{D}}[\hat{w}]=2 \pi H[w]
$$

Hence, $v$ minimizes $H$ and the uniqueness of $v$ follows from the uniqueness of $u$.

We will now be concerned with further properties of the 1-dimensional minimizer $v \in W^{1,1}(1,2)$ found in the previous lemma. We recall that functions $w \in W^{1,1}(1,2)$ have a continuous representative on the compact interval [1,2]. In the following we identify these functions with that representative and we will simply write $w(1)$ and $w(2)$ for the values of the trace as we already did in Lemma 3.7.

Lemma 3.8. Let $M \geqq 0$. The function v from Lemma 3.7 has the following properties:

$$
\begin{gathered}
0 \leqq v \leqq M, \\
v \text { is non-increasing, } \\
v(2)=0 .
\end{gathered}
$$

Proof. The proof rests on the fact that $v$ minimizes the functional $H$ defined in (3.8).

It is easily checked that $\tilde{v}:=\max \{\min \{v, M\}, 0\}$ satisfies $H[\tilde{v}] \leqq H[v]$. By the uniqueness assertion in Lemma 3.7 we thus get $\tilde{v}=v$ and (3.11).

To prove (3.12) we fix $t_{0} \in[1,2]$ and define

$$
\tilde{v}(t):= \begin{cases}v(t) & \text { for } t \leqq t_{0}, \\ \min \left\{v(t), v\left(t_{0}\right)\right\} & \text { for } t \geqq t_{0} .\end{cases}
$$

It is not difficult to show $H[\tilde{v}] \leqq H[v]$ (using $v \geqq 0$ ), and exploiting the uniqueness of $v$ again we find $\tilde{v}=v$. In other words this means $v(t) \leqq v\left(t_{0}\right)$ for $t \geqq t_{0}$ and (3.12) is proved.

Finally, we compare $v$ with $\tilde{v}:=v-v(2)$. Keeping in mind that we already know $v \geqq v(2) \geqq 0$ from (3.11) and (3.12) it can be checked that

$$
H[\tilde{v}] \leqq H[v]-|v(2)|
$$

holds. Since $v$ is minimizing, we thus get (3.13).

Lemma 3.9. Let $M \geqq 0$. We consider the function $v$ from Lemma 3.7 and define $v_{\varepsilon} \in W^{1,1}(1,2)$ by

$$
v_{\varepsilon}(t):= \begin{cases}v(t+\varepsilon) & \text { for } t \leqq 2-\varepsilon, \\ 0 & \text { for } t \geqq 2-\varepsilon .\end{cases}
$$

Then for $0<\varepsilon \leqq 2$ we have

$$
\begin{array}{r}
H\left[v_{\varepsilon}\right] \leqq H[v]-\varepsilon(1-\varepsilon / 2)(1-\log 2) v(1+\varepsilon)+2 \varepsilon . \\
\text { Bereitgestellt von | Universitaetsbibliothek Augsburg } \\
\text { Angemeldet } \\
\text { Heruntergeladen am | 04.02.19 08:18 }
\end{array}
$$


Proof. By Lemma 3.8 we have $v(2)=0$ and thus $v_{\varepsilon}$ is continuous at $2-\varepsilon$ and in $W^{1,1}(1,2)$. Moreover, using the definition of $H$ in (3.8) and the definition of $v_{\varepsilon}$ we have

$$
\begin{aligned}
H\left[v_{\varepsilon}\right] & =\int_{1}^{2} \sqrt{t^{2}\left(1+v_{\varepsilon}^{\prime}(t)^{2}\right)+v_{\varepsilon}(t)^{2}} d t+\left|v_{\varepsilon}(1)-M\right| \\
& \leqq \int_{1+\varepsilon}^{2} \sqrt{(t-\varepsilon)^{2}\left(1+v^{\prime}(t)^{2}\right)+v(t)^{2}} d t+2 \varepsilon+|v(1+\varepsilon)-M|
\end{aligned}
$$

Noting

$$
\begin{aligned}
|v(1+\varepsilon)-M| & \leqq|v(1)-M|+\int_{1}^{1+\varepsilon}\left|v^{\prime}(t)\right| d t \\
& \leqq|v(1)-M|+\int_{1}^{1+\varepsilon} t \sqrt{1+v^{\prime}(t)^{2}+t^{-2} v(t)^{2}} d t
\end{aligned}
$$

we thus get

$$
\begin{aligned}
H\left[v_{\varepsilon}\right] & \leqq H[v]-\int_{1+\varepsilon}^{2}\left[\sqrt{t^{2}\left(1+v^{\prime}(t)^{2}\right)+v(t)^{2}}-\sqrt{(t-\varepsilon)^{2}\left(1+v^{\prime}(t)^{2}\right)+v(t)^{2}}\right] d t+2 \varepsilon \\
& =H[v]-\int_{1+\varepsilon}^{2} \frac{\left(2 \varepsilon t-\varepsilon^{2}\right)\left(1+v^{\prime}(t)^{2}\right)}{\sqrt{(t-\varepsilon)^{2}\left(1+v^{\prime}(t)^{2}\right)+v(t)^{2}}+\sqrt{t^{2}\left(1+v^{\prime}(t)^{2}\right)+v(t)^{2}}} d t+2 \varepsilon \\
& =: H[v]-I+2 \varepsilon .
\end{aligned}
$$

Next we estimate

$$
I \geqq \int_{1+\varepsilon}^{2} \frac{\left(2 \varepsilon t-\varepsilon^{2}\right)\left(1+v^{\prime}(t)^{2}\right)}{2 \sqrt{t^{2}\left(1+v^{\prime}(t)^{2}\right)+v(t)^{2}}} d t \geqq\left(\varepsilon-\varepsilon^{2} / 2\right) I I,
$$

where we introduced

$$
I I:=\int_{1+\varepsilon}^{2} \frac{1+v^{\prime}(t)^{2}}{\sqrt{1+v^{\prime}(t)^{2}+t^{-2} v(t)^{2}}} d t .
$$

Exploiting the properties from Lemma 3.8 we control $I I$ as follows:

$$
\begin{aligned}
I I & =\int_{1+\varepsilon}^{2} \sqrt{1+v^{\prime}(t)^{2}+t^{-2} v(t)^{2}} d t-\int_{1+\varepsilon}^{2} \frac{t^{-2} v(t)^{2}}{\sqrt{1+v^{\prime}(t)^{2}+t^{-2} v(t)^{2}}} d t \\
& \geqq \int_{1+\varepsilon}^{2}\left|v^{\prime}(t)\right| d t-\int_{1+\varepsilon}^{2} \frac{v(t)}{t} d t
\end{aligned}
$$




$$
\begin{aligned}
& \geqq v(2)-v(1+\varepsilon)-v(1+\varepsilon) \int_{1}^{2} \frac{d t}{t} \\
& =(1-\log 2) v(1+\varepsilon) .
\end{aligned}
$$

Collecting the estimates we end up with (3.14).

Proof of Theorem 1.5. It suffices to treat the case $M \geqq 0$, for which Lemma 3.8 and Lemma 3.9 are available. We denote by $v$ the function from Lemma 3.7 and recall that we work with a continuous representative of $v$ on $[1,2]$. Assuming $v(1)>2 /(1-\log 2)$, we may find an $\varepsilon>0$ such that $(1-\varepsilon / 2)(1-\log 2) v(1+\varepsilon)>2$. Then Lemma 3.9 gives $H\left[v^{\varepsilon}\right]<H[v]$ for this $\varepsilon$, which contradicts the minimizing property of $v$. Consequently, we must have $v(1) \leqq 2 /(1-\log 2)$ and by Lemma 3.8 we deduce

$$
0 \leqq v \leqq \frac{2}{1-\log 2}
$$

Recalling $u(x)=v(|x|) x /|x|$ the claim follows.

Remark 3.10. The bound $2 /(1-\log 2)$ in Theorem 1.5 is not optimal and some refinements are possible. For instance an improved bound can be obtained using the Jensen type inequality

$$
\int_{1+\varepsilon}^{2} \sqrt{1+v^{\prime}(t)^{2}} d t \geqq \sqrt{(1-\varepsilon)^{2}+(v(2)-v(1+\varepsilon))^{2}}
$$

in the estimation of $I I$ in (3.15). However, we do not know how an optimal bound can be reached and we abandon the discussion of further refinements.

3.3. Santi's example, revisited. In this subsection we will revisit Santi's example from [74]. Arguing as in Section 3.2 we will provide similar examples-still in the scalar case $N=1$-involving a more general class of integrands. This generalization of the example will be useful in Section 3.4.

We start recalling that the construction of [74] works on two-dimensional domains

$$
\left\{x \in B_{l}^{2}: x \notin \overline{B_{r}^{2}( \pm l, \pm l)} \text { for the four possible choices of signs }\right\}
$$

where $l$ and $r$ are positive parameters such that $l<r<\sqrt{2} l$. These domains are axially symmetric, starshaped Lipschitz-domains containing the origin and bounded by four circular arcs; see Figure 1. For the purposes of Proposition 6.4 below it will be convenient to work with a suitable one-parameter-family of domains. Thus we choose $l=r / \sqrt{2}+1 / 4$, which is possible for all $r \geqq 1$, and denote the corresponding domain in (3.16) by $\Omega_{S}^{r}$. For the moment it suffices to deal with $\Omega_{S}:=\Omega_{S}^{1}$. 


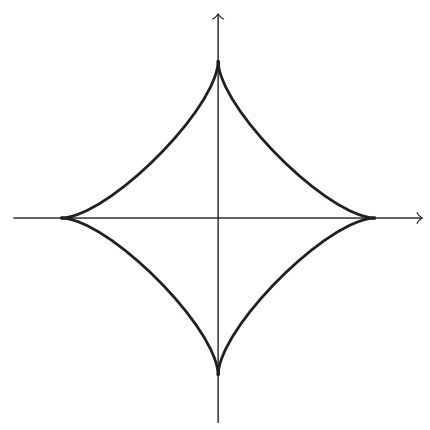

Figure 1. Santi's domain.

On $\partial \Omega_{S}$ we consider the piecewise constant boundary values $x \mapsto M \operatorname{sgn}\left(x_{1} x_{2}\right)$, where $M \geqq 0$ is fixed. In other words we choose some $u_{0} \in W^{1,1}\left(\Omega_{S}\right)$ such that

$$
u_{0}(x)=M \operatorname{sgn}\left(x_{1} x_{2}\right) \text { for } \mathscr{H}^{1} \text {-a.e. } x \in \partial \Omega_{S},
$$

and we let $\mathscr{D}:=u_{0}+W_{0}^{1,1}\left(\Omega_{S}\right)$. Then we have:

Proposition 3.11. Let $n=2, N=1$, and $\Omega=\Omega_{S}$. Assume that a $C^{2}$-integrand $f: \mathbb{R}^{2} \rightarrow[0, \infty)$ satisfies $(\mathrm{H} 1),(\mathrm{H} 2)$, and

$$
f(z)=\tilde{f}(|z|) \geqq f^{\infty}(z)=|z| \quad \text { for all } z \in \mathbb{R}^{2}
$$

and some $\tilde{f}:[0, \infty) \rightarrow[0, \infty)$. Then there exists a generalized minimizer $\hat{u} \in W^{1,1}\left(\Omega_{S}\right)$ of $F$ in $\mathscr{D}$ which is bounded independently of $M$, precisely

$$
\sup _{\Omega_{S}}|\hat{u}| \leqq 2 f(0)
$$

In particular, if $M>2 f(0)$ holds, then $\hat{u}+y$ is a generalized minimizer for every $y \in \mathbb{R}$ with $|y| \leqq M-2 f(0)$, and generalized minimizers of $F$ in $\mathscr{D}$ are not unique.

Following the idea of Santi [74] we will compare minimizers on $\Omega_{S}$ with minimizers on the two-dimensional annulus $B_{2}^{2} \backslash B_{1}^{2}$. Therefore, we look at the integral

$$
\int_{B_{2} \backslash B_{1}} f(\nabla w) d x+\int_{\partial B_{1}}|M-w| d \mathscr{H}^{1}+\int_{\partial B_{2}}|w| d \mathscr{H}^{1} \quad \text { for } w \in W^{1,1}(\Omega) .
$$

Analogously to Section 3.2 we have:

Lemma 3.12. Assume that $f$ is as in Proposition 3.11. Then there exists a unique minimizer $u$ of (3.19) among all scalar functions $w \in W^{1,1}(\Omega)$ and it can be written as

$$
u(x)=v(|x|) \quad \text { for } x \in \Omega
$$

Here, $v$ is the unique minimizer in $W^{1,1}(1,2)$ of the 1-dimensional scalar integral $H$, defined by

$$
H[w]:=\int_{1}^{2} \tilde{f}\left(\left|w^{\prime}(t)\right|\right) t d t+|w(1)-M|+2|w(2)| \quad \text { for } w \in W^{1,1}(1,2) .
$$


We omit the proof of Lemma 3.12 which exploits the radial symmetry of the boundary values and is similar to the proof of Lemma 3.7.

Lemma 3.13. Assume that $f$ is as in Proposition 3.11. Then the unique minimizer $u$ of (3.19) is bounded independently of $M$, precisely

$$
0 \leqq u \leqq 2 f(0) \quad \text { on } B_{2} \backslash B_{1} .
$$

Proof. We proceed as for Theorem 1.5 in Section 3.2. We first note that Lemma 3.8 carries over to the function $v$ in Lemma 3.12 with the same proof. Thus, $v$ is nonnegative and non-increasing with $v(2)=0$. Now for an arbitrary $\varepsilon>0$ we may use the comparison function $v_{\varepsilon}$ from Lemma 3.9 in the present situation. We have

$$
\begin{aligned}
H\left[v_{\varepsilon}\right] & =\int_{1}^{2} \tilde{f}\left(\left|v_{\varepsilon}^{\prime}(t)\right|\right) t d t+\left|v_{\varepsilon}(1)-M\right| \\
& \leqq \int_{1+\varepsilon}^{2} \tilde{f}\left(\left|v^{\prime}(t)\right|\right)(t-\varepsilon) d t+|v(1+\varepsilon)-M|+2 \varepsilon \tilde{f}(0) \\
& \leqq \int_{1+\varepsilon}^{2} \tilde{f}\left(\left|v^{\prime}(t)\right|\right) t d t+\int_{1}^{1+\varepsilon}\left|v^{\prime}(t)\right| d t+|v(1)-M|+\varepsilon\left[2 \tilde{f}(0)-\int_{1+\varepsilon}^{2} \tilde{f}\left(\left|v^{\prime}(t)\right|\right) d t\right] \\
& \leqq H[v]+\varepsilon\left[2 \tilde{f}(0)-\int_{1+\varepsilon}^{2}\left|v^{\prime}(t)\right| d t\right]
\end{aligned}
$$

where we exploited in the last estimate that $\tilde{f}(s) \geqq s$ holds by (3.18). Since $v$ minimizes $H$, the term in square brackets in the last line must be nonnegative, that is,

$$
v(1+\varepsilon) \leqq 2 \tilde{f}(0)=2 f(0)
$$

Recalling $u(x)=v(|x|)$ we arrive at the claim.

After these preparations we now establish Proposition 3.11. We remark that-with the preceding lemmas at hand-the remaining arguments are close to [74]. Nevertheless, for convenience of the reader we provide a proof in our terminology.

Proof of Proposition 3.11. We first apply Corollary 1.13 (recall that $(\mathrm{H} 3)$ holds trivially for $N=1$ ) which tells us that all generalized minimizers of $F$ are of class $W^{1,1}$. Then we start with an arbitrary generalized minimizer $w \in W^{1,1}\left(\Omega_{S}\right)$ of $F$ in $\mathscr{D}$ and we recall that the boundary values in (3.17) are odd in both variables $x_{1}$ and $x_{2}$. It follows that the minimizing property is preserved if we first pass from $w$ to $x \mapsto-w\left(-x_{1}, x_{2}\right)$ and then to the convex combination $x \rightarrow\left[w(x)-w\left(-x_{1}, x_{2}\right)\right] / 2$. The latter minimizer is odd in $x_{1}$, and by an analogous argument for $x_{2}$ we may find a generalized minimizer $\hat{u}$ of $F$ in $\mathscr{D}$ which is odd in both variables. This minimizer $\hat{u}$ will be fixed in the following and we record that it vanishes on both coordinate axes (in the sense of trace).

Next we consider the upper right quarter $\tilde{\Omega}_{S}$ of $\Omega_{S}$, that is,

$$
\tilde{\Omega}_{S}:=\left\{x \in \Omega_{S}: x_{1}>0, x_{2}>0\right\} .
$$


The boundary $\partial \tilde{\boldsymbol{\Omega}}_{S}$ is decomposed into a circular arc $\partial_{1} \tilde{\boldsymbol{\Omega}}_{S}$ and two line segments $\partial_{2} \tilde{\boldsymbol{\Omega}}_{S}$, and we notice that $\hat{u}$ has trace 0 on $\partial_{2} \tilde{\Omega}_{S}$. For an arbitrary $w \in W^{1,1}\left(\tilde{\Omega}_{S}\right)$ we now define $\tilde{w} \in B V\left(\Omega_{S}\right)$ by

$$
\tilde{w}(x):= \begin{cases}w(x) & \text { for } x \in \tilde{\Omega}_{S}, \\ \hat{u}(x) & \text { for } x \in \Omega_{S} \backslash \tilde{\boldsymbol{\Omega}}_{S}\end{cases}
$$

and observe $\mathscr{F}^{\mathscr{D}}[\hat{u}] \leqq \mathscr{F}^{\mathscr{D}}[\tilde{w}]$, by the minimizing property of $\hat{u}$. However, some terms in this inequality cancel out and we find that the restriction of $\hat{u}$ to $\tilde{\Omega}_{S}$ minimizes

$$
\int_{\tilde{\Omega}_{S}} f(\nabla w) d x+\int_{\partial_{1} \tilde{\Omega}_{S}}|M-w| d \mathscr{H}^{1}+\int_{\partial_{2} \tilde{\Omega}_{S}}|w| d \mathscr{H}^{1}
$$

among all $w \in W^{1,1}\left(\tilde{\boldsymbol{\Omega}}_{S}\right)$.

Now we come back to the nonnegative minimizer $u$ from Lemma 3.12 and Lemma 3.13. With a slight abuse of notation we shift the annulus in Lemma 3.12 in such a way that its center is the point $(1 / \sqrt{2}+1 / 4,1 / \sqrt{2}+1 / 4)$ but still denote it by $B_{2} \backslash B_{1}$. Recalling the construction of $\Omega_{S}$ we have thus arranged $\tilde{\Omega}_{S} \subset B_{2} \backslash B_{1}$ and $\partial_{1} \tilde{\Omega}_{S} \subset \partial B_{1}$. Arguing in the same way as we did with $\hat{u}$ before we find that the restriction of $u$ to $\tilde{\Omega}_{S}$ minimizes

$$
\int_{\tilde{\Omega}_{S}} f(\nabla w) d x+\int_{\partial_{1} \tilde{\Omega}_{S}}|M-w| d \mathscr{H}^{1}+\int_{\partial_{2} \tilde{\Omega}_{S}}|u-w| d \mathscr{H}^{1}
$$

among all $w \in W^{1,1}\left(\tilde{\Omega}_{S}\right)$. The minimizing properties of $\hat{u}$ and $u$ on $\tilde{\Omega}_{S}$ enable us to apply the comparison principle from Lemma 2.10 . Recalling $u \geqq 0$ we come out with $\hat{u} \leqq u$ on $\tilde{\Omega}_{S}$ and taking Lemma 3.13 into account we arrive at

$$
\hat{u} \leqq 2 f(0) \quad \text { on } \tilde{\Omega}_{S}
$$

Moreover, by a minimum principle (which is a simple variant of those in Appendix D) we have $\hat{u} \geqq 0$ and thus $|\hat{u}| \leqq 2 f(0)$ on $\tilde{\Omega}_{S}$. By the symmetries of $\hat{u}$ this inequality holds on the whole domain $\Omega_{S}$ and we have obtained the claimed estimate which is independent of $M$.

Finally, let us assume that $M>2 f(0)$ holds, which implies that $\hat{u}$ is bounded away from $u_{0}$ on $\partial \Omega_{S}$. If we add to $\hat{u}$ some $y \in \mathbb{R}$ with $|y| \leqq M-2 f(0)$, then we increase the integrand of the boundary integral in (1.8) by $|y|$ on one half of $\partial \Omega_{S}$ and we decrease it by $|y|$ on the other half. Thus, we have $\mathscr{F}^{\mathscr{D}}[\hat{u}+y]=\mathscr{F}^{\mathscr{D}}[\hat{u}]$ and $\hat{u}+y$ is a generalized minimizer of $F$ in $\mathscr{D}$.

3.4. An $N$-parameter-family of minimizers. Returning to the vector-valued setting with an arbitrary $N \in \mathbb{N}$ we will now demonstrate that the assumption (H4) in Theorem 1.16 is inevitable. To this end we will apply the results of Section 3.1 to construct an integrand $f$ which satisfies $(\mathrm{H} 1),(\mathrm{H} 2)$, and (1.12), but for which $(\mathrm{H} 4)$ fails. Then we will show that the generalized minimizers of $F$ in a suitable Dirichlet class form an $N$-parameterfamily.

Indeed in the following construction we use the domain $\Omega_{S}$ from the beginning of Section 3.3 and the boundary values $x \mapsto\left(M \operatorname{sgn}\left(x_{1} x_{2}\right), 0, \ldots, 0\right)$ on $\partial \Omega_{S}$. As in Section 
3.3 we write $\mathscr{D}=u_{0}+W_{0}^{1,1}\left(\Omega_{S}\right)$ for the scalar Dirichlet class corresponding to a function $u_{0} \in W^{1,1}\left(\Omega_{S}\right)$ with $u_{0}(x)=M \operatorname{sgn}\left(x_{1} x_{2}\right)$ for $\mathscr{H}^{1}$-a.e. $x \in \partial \Omega_{s}$. Additionally, we introduce the vector-valued Dirichlet class

$$
\mathscr{D} \times\{0\}=\left(u_{0}, 0, \ldots, 0\right)+W_{0}^{1,1}\left(\Omega_{S}, \mathbb{R}^{N}\right) .
$$

With this terminology we may state:

Theorem 3.14. Let $n=2$ and $\Omega=\Omega_{S}$. We fix $\mu>2$ and the Dirichlet class $\mathscr{D} \times\{0\}$ from (3.20). If $M \geqq M_{0}$ holds for some positive constant $M_{0}$, depending only on $N$ and $\mu$, then there exist a smooth convex integrand $f: \mathbb{R}^{N 2} \rightarrow[0, \infty)$ and a generalized minimizer $\bar{u}$ of $F$ in $\mathscr{D} \times\{0\}$ with the following properties:

- (H1), (H2 $\left.{ }_{\mu}\right)$, and (1.12) are valid, but (H4) fails for $f$.

- For all $y \in \mathbb{R}^{N}$ with $|y| \leqq M / 2$ the function $\bar{u}+y$ is a generalized minimizer of $F$ in $\mathscr{D} \times\{0\}$.

Proof. It suffices to treat the case $2<\mu \leqq 3$ since $(\mathrm{H} 2 \mu)$ is a weaker condition when $\mu$ is larger. The bound $\mu \leqq 3$ will allow an application of Proposition 3.11.

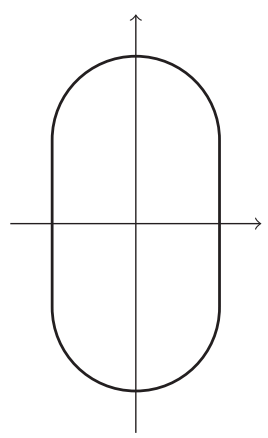

Figure 2. The unit ball of $\tilde{g}$.

Now we start defining $\tilde{g}: \mathbb{R}^{N} \rightarrow[0, \infty)$ by

$$
\tilde{g}(y):= \begin{cases}\left|y^{1}\right| & \text { for }\left|y^{\prime}\right| \leqq\left|y^{1}\right|, \\ \frac{|y|^{2}}{2\left|y^{\prime}\right|} & \text { for }\left|y^{\prime}\right|>\left|y^{1}\right|,\end{cases}
$$

where $y=\left(y^{1}, y^{\prime}\right) \in \mathbb{R} \times \mathbb{R}^{N-1}$. Then $\tilde{g}$ is 1 -homogeneous, convex, and locally $C^{1,1}$ on $\mathbb{R}^{N} \backslash\{0\}$. In fact, this properties can be seen looking only at the unit ball of $\tilde{g}$ which can be visualized for $N=2$ as the union of a square and two balls (see Figure 2) and in higher dimensions by rotating the two-dimensional picture. For our purposes it is crucial that this unit ball of $\tilde{g}$ is convex but not strictly convex. We now define $g: \mathbb{R}^{N 2} \rightarrow[0, \infty)$ by

$$
g(z):=\tilde{g}\left(\left|z^{1}\right|,\left|z^{2}\right|, \ldots,\left|z^{N}\right|\right),
$$


where $z^{\alpha} \in \mathbb{R}^{2}$ denotes the $\alpha$ th row of $z \in \mathbb{R}^{N 2}$ as in (1.12). Then $g$ is also 1-homogeneous, convex, and locally $C^{1,1}$ on $\mathbb{R}^{N 2} \backslash\{0\}$. Now we apply Proposition 3.1 to obtain a smooth integrand $f: \mathbb{R}^{N 2} \rightarrow[0, \infty)$ with $(\mathrm{H} 1),\left(\mathrm{H} 2{ }_{\mu}\right)$, and

$$
f \geqq f^{\infty}=g \quad \text { on } \mathbb{R}^{N 2}
$$

where the last inequality relies on the hypothesis $\mu>2$; compare Remark 3.2. Moreover, in view of Proposition 3.4 we may write

$$
f(z)=\tilde{f}\left(\left|z^{1}\right|,\left|z^{2}\right|, \ldots,\left|z^{N}\right|\right)
$$

for some function $\tilde{f}:[0, \infty)^{N} \rightarrow[0, \infty)$. Since only the first component function of the above boundary values does not vanish, we now concentrate on the first argument of $\tilde{f}$ : We introduce

$$
f_{\diamond}(\xi):=\tilde{f}(|\xi|, 0, \ldots, 0) \quad \text { for } \xi \in \mathbb{R}^{2}
$$

and the corresponding integral

$$
F_{\diamond}[w]:=\int_{\Omega_{S}} f_{\diamond}(\nabla w) d x
$$

for scalar functions $w$ on $\Omega_{S}$. Next we observe that $f$ is convex and even in each of its variables. Thus $z^{i} \mapsto f(z)$ attains its minimum for $z^{i}=0$ and we have the inequality

$$
f(z) \geqq f_{\diamond}\left(z^{1}\right) \quad \text { for } z \in \mathbb{R}^{N 2}
$$

which will be useful below. From the above construction and the corresponding properties of $f$ we deduce that $f_{\diamond}$ satisfies $(\mathrm{H} 1),\left(\mathrm{H} 2_{\mu}\right)$, and

$$
f_{\diamond}(\xi) \geqq\left(f_{\diamond}\right)^{\infty}(\xi)=\tilde{g}(|\xi|, 0, \ldots, 0)=|\xi| .
$$

In particular, (3.18) holds for $f_{\diamond}$ (in place of $f$ ) and we may apply Proposition 3.11 (remember that we assumed $\mu \leqq 3$ ) to the scalar integral $F_{\diamond}$. We come out with a generalized minimizer $\hat{u}$ of $F_{\diamond}$ in $\mathscr{D}$ such that

$$
\sup _{\Omega_{S}}|\hat{u}| \leqq 2 f_{\diamond}(0)=2 f(0)
$$

holds. Adding zero-components to $\hat{u}$ we define the $\mathbb{R}^{N}$-valued function

$$
\bar{u}:=(\hat{u}, 0, \ldots, 0)
$$

and we observe that for all $w=\left(w^{1}, w^{2}, \ldots, w^{N}\right) \in B V\left(\Omega_{S}, \mathbb{R}^{N}\right)$ it holds

$$
\mathscr{F}^{\mathscr{D} \times\{0\}}[\bar{u}]=\mathscr{F}_{\diamond}{ }^{\mathscr{D}}[\hat{u}] \leqq \mathscr{F}_{\diamond}{ }^{\mathscr{D}}\left[w^{1}\right] \leqq \mathscr{F}^{\mathscr{D} \times\{0\}}[w],
$$

where we used (3.21) to derive the last inequality. In particular, $\bar{u}$ is a generalized minimizer of $F$ in $\mathscr{D} \times\{0\}$. 
In order to construct more minimizers we consider an arbitrary $y \in \mathbb{R}^{N}$ with $|y| \leqq M / 2$. Then we have

$$
\left|u_{0}-\hat{u}-y_{1}\right|-\left|y^{\prime}\right| \geqq\left|u_{0}\right|-|\hat{u}|-\sqrt{2}|y| \geqq\left(1-\frac{\sqrt{2}}{2}\right) M-2 f(0) \quad \text { on } \partial \Omega_{S},
$$

where we decomposed $y=\left(y^{1}, y^{\prime}\right) \in \mathbb{R} \times \mathbb{R}^{N-1}$ as before. We choose $M_{0}$ large enough such that for $M \geqq M_{0}$ the right-hand side of the previous estimate is nonnegative, and we get

$$
\left|y^{\prime}\right| \leqq\left|u_{0}-\hat{u}-y_{1}\right| \quad \text { on } \partial \Omega_{s} .
$$

We record that the preceding choice of $M_{0}$ depends only on the construction of $f$, which in turn depends only on $N$ and $\mu$. Recalling (1.8) we observe that $\mathscr{F} \mathscr{D} \times\{0\}[\bar{u}+y]$ and $\mathscr{F}_{\diamond}^{\mathscr{D}}\left[\hat{u}+y^{1}\right]$ differ at most in the boundary integrals. Written out these integrals are

$$
\int_{\partial \Omega_{S}} \tilde{g}\left(u_{0}-\hat{u}-y^{1}, y^{\prime}\right) d \mathscr{H}^{1} \quad \text { and } \quad \int_{\partial \Omega_{S}}\left|u_{0}-\hat{u}-y^{1}\right| d \mathscr{H}^{1} .
$$

By (3.22) and the definition of $\tilde{g}$ these two quantities indeed coincide and hence we also have

$$
\mathscr{F}^{\mathscr{D}} \times\{0\}[\bar{u}+y]=\mathscr{F}_{\diamond}^{\mathscr{D}}\left[\hat{u}+y^{1}\right] .
$$

However, by the last part of Proposition 3.11 (note that $\left|y^{1}\right| \leqq M / 2 \leqq M-2 f(0)$ by the choice of $M_{0}$ ) we know that $\hat{u}+y^{1}$ is a generalized minimizer of $F_{\diamond}$, and thus we moreover have

$$
\mathscr{F}_{\diamond}^{\mathscr{D}}\left[\hat{u}+y^{1}\right]=\mathscr{F}_{\diamond}^{\mathscr{D}}[\hat{u}]
$$

Collecting the above equalities we come out with

$$
\mathscr{F}^{\mathscr{D}} \times\{0\}[\bar{u}+y]=\mathscr{F} \mathscr{D} \times\{0\}[\bar{u}] .
$$

Consequently, $\bar{u}+y$ is a generalized minimizer of $F$ in $\mathscr{D} \times\{0\}$ for all $y \in \mathbb{R}^{N}$ with $|y| \leqq M / 2$.

We close this section with a comment on the hypothesis $\mu>2$.

Remark 3.15. The assumption $\mu>2$ in Theorem 3.14 is related to Serrin's classification of non-uniformly elliptic equations from [80]. In particular, Serrin showed that the classical Dirichlet problem for equations with a well-defined Bernstein genre $g_{B}$ is generally solvable if and only if one has $g_{B} \leqq 1$. On the contrary, for $g_{B}>1$ general solvability fails if a part of the boundary has negative generalized mean curvature. In our setting the same phenomenon occurs. In fact, $\mu$ essentially corresponds to $g_{B}+1$, and revisiting the arguments of this section we see that $\mu>2$ was needed to construct an integrand with (3.18); compare Remark 3.2. In turn (3.18) was exploited in Lemma 3.13 which gives (for large 
$M)$ non-attainment of the boundary values on a negatively curved part of boundary. Finally, this non-attainment implies that the classical Dirichlet problem is not solvable.

\section{Local boundedness}

In this section we will prove Theorem 1.11. Let us briefly sketch the proof. We will start with an approximation procedure based on the application of Ekeland's variational principle in the Dirichlet class $\mathscr{D}=u_{0}+W_{0}^{1,1}\left(\Omega, \mathbb{R}^{N}\right)$. Then exploiting the structure condition (H3) we will use Moser's iteration technique to obtain interior $L^{p(k)}$-estimates for the functions $u_{k}$ in a minimizing sequence. Since the exponents $p(k)$ tend to $\infty$, we may deduce the claimed $L^{\infty}$-estimate for minimizers $u$.

We assume that the hypotheses of Theorem 1.11 are valid, and we fix a generalized minimizer $u \in B V\left(\Omega, \mathbb{R}^{N}\right)$ of $F$ in $\mathscr{D}$. Then by Theorem 1.8 there exists a minimizing sequence $\left(w_{k}\right)_{k \in \mathbb{N}}$ for $F$ in $\mathscr{D}$ such that $w_{k}$ converges to $u$ in $L^{1}\left(\Omega, \mathbb{R}^{N}\right)$. Passing possibly to a subsequence we may assume

$$
F\left[w_{k}\right] \leqq \inf _{\mathscr{D}} F+\frac{1}{k^{2}}
$$

Next we will apply Lemma 2.11 to the functional $F$ on the Dirichlet class $\mathscr{D}$. Here, $\mathscr{D}$ is endowed with the metric $d_{\mathscr{D}}$ defined by

$$
d_{\mathscr{D}}(u, v):=\int_{\Omega}|\nabla u-\nabla v| d x \quad \text { for } u, v \in \mathscr{D} .
$$

With respect to this metric the semicontinuity assumption in Lemma 2.11 is satisfied as a consequence of Fatou's lemma. Applying Lemma 2.11 to each $w_{k}$ we get functions $u_{k} \in \mathscr{D}$ such that

$$
\begin{gathered}
d_{\mathscr{D}}\left(u_{k}, w_{k}\right) \leqq \frac{1}{k} \\
F\left[u_{k}\right] \leqq F[w]+\frac{1}{k} d_{\mathscr{D}}\left(u_{k}, w\right) \quad \text { for all } w \in \mathscr{D}
\end{gathered}
$$

In particular, (4.1) implies by Poincaré's inequality that $u_{k}-w_{k}$ converges to 0 strongly in $W^{1,1}\left(\Omega, \mathbb{R}^{N}\right)$ and thus we have the convergence

$$
u_{k} \underset{k \rightarrow \infty}{\longrightarrow} u \text { strongly in } L^{1}\left(\Omega, \mathbb{R}^{N}\right)
$$

to the given minimizer $u$. For every $\varphi \in W_{0}^{1,1}\left(\Omega, \mathbb{R}^{N}\right)$ we know by (4.2) that the function

$$
\mathbb{R} \rightarrow \mathbb{R}, \quad a \mapsto F\left[u_{k}+a \varphi\right]+\frac{|a|}{k} \int_{\Omega}|\nabla \varphi| d x
$$


has a minimum at 0 . Writing down the first-order criteria for this minimality we have

$$
\begin{aligned}
& \left.\frac{d}{d a}\right|_{a=0+}\left[F\left[u_{k}+a \varphi\right]+\frac{a}{k} \int_{\Omega}|\nabla \varphi| d x\right] \geqq 0, \\
& \left.\frac{d}{d a}\right|_{a=0-}\left[F\left[u_{k}+a \varphi\right]-\frac{a}{k} \int_{\Omega}|\nabla \varphi| d x\right] \leqq 0 .
\end{aligned}
$$

Computing the derivatives we then end up with the perturbed Euler equation

$$
\left|\int_{\Omega} \nabla f\left(\nabla u_{k}\right) \cdot \nabla \varphi d x\right| \leqq \frac{1}{k} \int_{\Omega}|\nabla \varphi| d x \quad \text { for all } \varphi \in W_{0}^{1,1}\left(\Omega, \mathbb{R}^{N}\right) .
$$

In the following lemmas we will implement the announced variant of Moser's iteration technique, permanently assuming that the hypotheses of Theorem 1.11 hold. In particular, we will use the structure condition (H3). we have

Lemma 4.1. We fix $t \geqq 1$ and suppose that (4.4) holds for $u_{k} \in \mathscr{D}$ with $k \geqq 2 t / \gamma$. Then

$$
\left|u_{k}\right|^{t} \in L_{\mathrm{loc}}^{1}(\Omega) \Rightarrow\left|u_{k}\right|^{t} \in W_{\mathrm{loc}}^{1,1}(\Omega)
$$

and moreover for every $s \geqq 1$ and every $\eta \in C_{\mathrm{cpt}}^{\infty}(\Omega)$ with $M_{\eta}:=\max _{\Omega}|\nabla \eta|>0$ the following Caccioppoli type estimate holds true:

$$
\int_{\Omega}\left|\nabla\left(\eta^{s}\left|u_{k}\right|^{t}\right)\right| d x \leqq C\left(t^{2}+s\right) t M_{\eta}\left[M_{\eta}^{-t} \lambda^{t} t^{-t} \int_{\Omega} \eta^{t+s-1} d x+\int_{\Omega} \eta^{s-1}\left|u_{k}\right|^{t} d x\right] .
$$

Here, $C$ depends only on $N n, \gamma$, and $\Gamma$.

Proof. We first recall that by Lemma 2.7 and Lemma 2.8 we have

$$
\begin{gathered}
|\nabla f(z)| \leqq C, \\
\nabla f(z) \cdot z \geqq \gamma|z|-\lambda .
\end{gathered}
$$

We define for $H>0$ the truncation operator $T_{H} y:=\min \{y, H\}$. Setting

$$
\varphi:=\eta^{s}\left(T_{H}\left|u_{k}\right|\right)^{t-1} u_{k}
$$

we compute

$$
\begin{aligned}
\nabla \varphi= & s \eta^{s-1}\left(T_{H}\left|u_{k}\right|\right)^{t-1} u_{k} \otimes \nabla \eta+\eta^{s}\left(T_{H}\left|u_{k}\right|\right)^{t-1} \nabla u_{k} \\
& +\eta^{s}(t-1)\left|u_{k}\right|^{t-3} u_{k} \otimes\left(u_{k}^{T} \nabla u_{k}\right) 1_{\left\{\left|u_{k}\right| \leqq H\right\}}
\end{aligned}
$$

(where the right-hand side is to be understood as 0 at the zeros of $u_{k}$ ) and

$$
|\nabla \varphi| \leqq s \eta^{s-1}\left(T_{H}\left|u_{k}\right|\right)^{t-1}\left|u_{k}\right||\nabla \eta|+t \eta^{s}\left(T_{H}\left|u_{k}\right|\right)^{t-1}\left|\nabla u_{k}\right|
$$

In particular, we infer $\varphi \in W_{0}^{1,1}\left(\Omega, \mathbb{R}^{N}\right)$ and thus we may use $\varphi$ as a test function in (4.4). Rearranging the terms and using (4.5) we come out with 


$$
\begin{aligned}
& \int_{\Omega} \eta^{s}\left[\left(T_{H}\left|u_{k}\right|\right)^{t-1} \nabla f\left(\nabla u_{k}\right) \cdot \nabla u_{k}+(t-1)\left|u_{k}\right|^{t-3}\left(u_{k}^{T} \nabla f\left(\nabla u_{k}\right)\right) \cdot\left(u_{k}^{T} \nabla u_{k}\right) \mathbb{1}_{\left\{\left|u_{k}\right| \leqq H\right\}}\right] d x \\
& \quad \leqq C s \int_{\Omega} \eta^{s-1}\left|u_{k}\right|^{t}|\nabla \eta| d x+\frac{1}{k} \int_{\Omega}|\nabla \varphi| d x
\end{aligned}
$$

Next we use (4.6) for the first and (H3) for the second term on the left-hand side. We shift the terms containing $\lambda$ to the right-hand side and get

$$
\gamma \int_{\Omega} \eta^{s}\left(T_{H}\left|u_{k}\right|\right)^{t-1}\left|\nabla u_{k}\right| d x \leqq \lambda t \int_{\Omega} \eta^{s}\left|u_{k}\right|^{t-1} d x+C s \int_{\Omega} \eta^{s-1}\left|u_{k}\right|^{t}|\nabla \eta| d x+\frac{1}{k} \int_{\Omega}|\nabla \varphi| d x
$$

Employing (4.7) we remove the remaining occurrence of $\nabla \varphi$ on the right-hand side and find

$$
\begin{aligned}
\gamma \int_{\Omega} \eta^{s}\left(T_{H}\left|u_{k}\right|\right)^{t-1}\left|\nabla u_{k}\right| d x \leqq & \lambda t \int_{\Omega} \eta^{s}\left|u_{k}\right|^{t-1} d x+C s \int_{\Omega} \eta^{s-1}\left|u_{k}\right|^{t}|\nabla \eta| d x \\
& +\frac{t}{k} \int_{\Omega} \eta^{s}\left(T_{H}\left|u_{k}\right|\right)^{t-1}\left|\nabla u_{k}\right| d x
\end{aligned}
$$

Thus, for $k \geqq 2 t / \gamma$ we may absorb the last term, and passing to the limit $H \rightarrow \infty$ via Fatou's lemma, we arrive at

$$
\int_{\Omega} \eta^{s}\left|u_{k}\right|^{t-1}\left|\nabla u_{k}\right| d x \leqq C\left[\lambda t \int_{\Omega} \eta^{s}\left|u_{k}\right|^{t-1} d x+s M_{\eta} \int_{\Omega} \eta^{s-1}\left|u_{k}\right|^{t} d x\right] .
$$

By the inequality

$$
\left|\nabla\left(\eta^{s}\left|u_{k}\right|^{t}\right)\right| \leqq t \eta^{s}\left|u_{k}\right|^{t-1}\left|\nabla u_{k}\right|+s M_{\eta} \eta^{s-1}\left|u_{k}\right|^{t}
$$

we deduce

$$
\int_{\Omega}\left|\nabla\left(\eta^{s}\left|u_{k}\right|^{t}\right)\right| d x \leqq C\left(t^{2}+s\right)\left[\lambda \int_{\Omega} \eta^{s}\left|u_{k}\right|^{t-1} d x+t M_{\eta} \int_{\Omega} \eta^{s-1}\left|u_{k}\right|^{t} d x\right] .
$$

A final application of Young's inequality gives the claimed estimate. equality:

Combining Lemma 4.1 with Sobolev's embedding we deduce a reverse Hölder in-

Lemma 4.2. We fix $t \geqq 1$ and suppose that (4.4) holds for $u_{k} \in \mathscr{D}$ with $k \geqq 2 t / \gamma$. Then

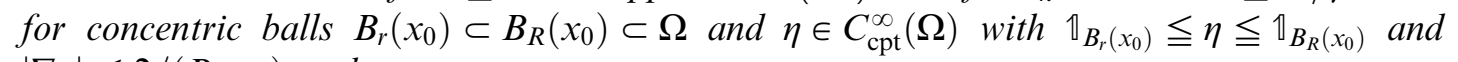
$|\nabla \eta| \leqq 2 /(R-r)$ we have

$$
\begin{aligned}
& {\left[\frac{1}{R^{n}} \int_{B_{R}\left(x_{0}\right)}\left(\eta^{n}\left|u_{k}\right|\right)^{\frac{n}{n-1} t} \eta^{-n} d x\right]^{\frac{n-1}{n}}} \\
& \quad \leqq C_{P} \frac{R t^{3}}{R-r}\left[(R-r)^{t} \lambda^{t} t^{-t}+\frac{1}{R^{n}} \int_{B_{R}\left(x_{0}\right)}\left(\eta^{n}\left|u_{k}\right|\right)^{t} \eta^{-n} d x\right]
\end{aligned}
$$

with a constant $C_{P} \geqq 1$, depending only on $n, N, \gamma$, and $\Gamma$. 
Proof. We set $s:=1-n+n t$ and note $1 \leqq s \leqq n t$. With this choice of $s$ the claim follows from the previous lemma by Sobolev's embedding.

Proof of Theorem 1.11. We will iterate the inequality in Lemma 4.2. To this aim we introduce for $j \in \mathbb{N} \cup\{0\}$ and $k \in \mathbb{N}$ the abbreviations

$$
\begin{aligned}
t_{j} & :=\left(\frac{n}{n-1}\right)^{j}, \\
\Psi_{k}(j) & :=\left[\frac{1}{R^{n}} \int_{B_{R}\left(x_{0}\right)}\left(\eta^{n}\left|u_{k}\right|\right)^{t_{j}} \eta^{-n} d x\right]^{\frac{1}{t_{j}}}, \\
A_{j} & :=\left(\frac{C_{P} R t_{j}^{3}}{R-r}\right)^{\frac{1}{t_{j}}},
\end{aligned}
$$

where $C_{P}$ denotes the constant from Lemma 4.2. With this terminology the estimate in Lemma 4.2 reads

$$
\Psi_{k}(j+1) \leqq A_{j}\left[\frac{R-r}{t_{j}} \lambda+\Psi_{k}(j)\right] \text { provided that } k \geqq \frac{2 t_{j}}{\gamma},
$$

and iterating this inequality we conclude for $m \in \mathbb{N}$

$$
\Psi_{k}(m+1) \leqq \sum_{l=0}^{m}\left(\prod_{j=l}^{m} A_{j}\right) \frac{R-r}{t_{l}} \lambda+\left(\prod_{j=0}^{m} A_{j}\right) \Psi_{k}(0) \quad \text { provided that } k \geqq \frac{2 t_{m}}{\gamma} .
$$

At this stage we may pass to the limit $k \rightarrow \infty$. To this end we define $\Psi(j)$ analogous to $\Psi_{k}(j)$, but with $u$ instead of $u_{k}$. Then using Fatou's lemma on the left-hand side and the strong convergence in (4.3) on the right-hand side, we may omit the indices $k$ in the last formula. Since the infinite product

$$
\prod_{j=0}^{\infty} A_{j}=\left(\frac{C_{P} R}{R-r}\right)^{\sum_{j=0}^{\infty}\left(\frac{n-1}{n}\right)^{j}} \prod_{j=0}^{\infty} t_{j}^{\frac{3}{t_{j}}}=\left(\frac{C_{P} R}{R-r}\right)^{n}\left(\frac{n}{n-1}\right)^{3 \sum_{j=0}^{\infty} j\left(\frac{n-1}{n}\right)^{j}}
$$

converges, we infer

$$
\Psi(m+1) \leqq C\left(\frac{R}{R-r}\right)^{n}\left[(R-r) \lambda \sum_{l=0}^{\infty}\left(\frac{n-1}{n}\right)^{l}+\Psi(0)\right] .
$$

Passing $m \rightarrow \infty$ and applying Lemma 2.1 we end up with

$$
\sup _{B_{r}\left(x_{0}\right)}|u| \leqq C\left(\frac{R}{R-r}\right)^{n}\left[(R-r) \lambda+\frac{1}{R^{n}} \int_{B_{R}\left(x_{0}\right)}|u| d x\right] .
$$

\section{Uniqueness}

Up to the end of Section 5.3 we will impose the hypotheses of Theorem 1.10 on the integrand $f$ and we will consider bounded generalized minimizers of the integral $F$ in (1.1) 
which are a priori in the space $B V\left(\Omega, \mathbb{R}^{N}\right) \cap L^{\infty}\left(\Omega, \mathbb{R}^{N}\right)$. If a boundedness condition for an approximating sequence is imposed, then by a result of [15] there exists one such minimizer which is in fact in $W^{1,1}\left(\Omega, \mathbb{R}^{N}\right)$, with an additional $L \log L$-bound for the derivative. Basically following the estimates of [15] we will now prove that this regularity result is valid for each minimizer.

Let us briefly sketch our line of argument. As in Section 4 we apply Ekeland's variational principle in Section 5.1 to construct a minimizing sequence $\left(u_{k}\right)_{k \in \mathbb{N}}$ which stays close to the given minimizer $u$. However, this approach leads to the occurrence of an additional perturbation term. We will show that it is convenient-in particular for the purposes of Section 5.2-to apply Ekeland's principle in the Sobolev space $W^{-1,1}$ leading to a rather harmless ${ }^{14)}$ perturbation. We remark that even though Ekeland's principle is nowadays a standard tool, this particular way of applying seems to be new.

Proceeding with the proof we exploit that $u$ is in $L^{\infty}$ via a suitable regularization procedure, which is partially inspired by arguments of [26]. In this way we derive some uniform exponential integrability for the sequence $u_{k}$. In the next step we establish estimates involving $\nabla^{2} u_{k}$ and then we provide uniform $L \log L$-estimates for $\nabla u_{k}$. In Section 5.3 we complete the regularity proof, and we deduce Theorem 1.10 and Corollary 1.13. Finally, Section 5.4 is devoted to the proof of Theorem 1.16 and Theorem 1.3.

Now let us go into the details.

First we observe that $\nabla f$ is bounded by Lemma 2.7 and thus $F$ is Lipschitz, that is,

$$
|F[\tilde{w}]-F[w]| \leqq L\|\tilde{w}-w\|_{W^{1,1}\left(\Omega, \mathbb{R}^{N}\right)} \quad \text { for all } w, \tilde{w} \in W^{1,1}\left(\Omega, \mathbb{R}^{N}\right)
$$

where the constant $L$ depends only on $N n$ and $\Gamma$ and is fixed for the remainder of the section.

For the purpose of proving regularity we fix an arbitrarily given bounded generalized minimizer $u$ of $F$ in a Dirichlet class, say $\tilde{\mathscr{D}}=\tilde{u}_{0}+W_{0}^{1,1}\left(\Omega, \mathbb{R}^{N}\right)$. Setting

$$
M:=\sup _{\Omega}|u|
$$

we then have

$$
u \in B V\left(\Omega, \mathbb{R}^{N}\right) \cap L_{M}^{\infty}\left(\Omega, \mathbb{R}^{N}\right)
$$

(see (2.1) for the definition of $L_{M}^{\infty}$ ). By Gagliardo's result [40], Teorema 1.II, and a cut-off argument there exists a function

$$
u_{0} \in W^{1,1}\left(\Omega, \mathbb{R}^{N}\right) \cap L_{M}^{\infty}\left(\Omega, \mathbb{R}^{N}\right)
$$

14) We could work with even weaker perturbations. Actually, instead of $W^{-1,1}\left(\Omega, \mathbb{R}^{N}\right)$ we might employ every complete metric space into which $W^{-1,1}\left(\Omega, \mathbb{R}^{N}\right)$ is continuously embedded. 
which coincides with $u$ on $\partial \Omega$ in the sense of trace. By Lemma 2.9, $u$ minimizes with respect to its own boundary values, precisely $u$ is a generalized minimizer of $F$ not only in $\tilde{\mathscr{D}}$ but also in

$$
\mathscr{D}:=u_{0}+W_{0}^{1,1}\left(\Omega, \mathbb{R}^{N}\right) .
$$

5.1. Regularization and approximation. In this subsection we implement the announced approximation procedure relying on the application of Lemma 2.11 in $W^{-1,1}$.

By Lemma 2.3 there exists a sequence $\left(w_{k}\right)_{k \in \mathbb{N}}$ in $\mathscr{D}$ such that

$$
w_{k} \text { converges to } u \text { in } L^{1}\left(\Omega, \mathbb{R}^{N}\right)
$$

and $\left(\mathscr{L}^{n}, D w_{k}\right)$ converges strictly to $\left(\mathscr{L}^{n}, D u\right)$ in the sense of measures on $\Omega$. Moreover, as the following argument shows we may assume that $\left(w_{k}\right)_{k \in \mathbb{N}}$ is a sequence in $L_{M}^{\infty}\left(\Omega, \mathbb{R}^{N}\right)$.

Indeed, if $\left(w_{k}\right)_{k \in \mathbb{N}}$ were not in $L_{M}^{\infty}\left(\Omega, \mathbb{R}^{N}\right)$, we would replace it by the truncated sequence $\left(\tilde{w}_{k}\right)_{k \in \mathbb{N}}$ defined by

$$
\tilde{w}_{k}(x):=\left\{\begin{array}{ll}
w_{k}(x) & \text { if }\left|w_{k}(x)\right| \leqq M, \\
\frac{w_{k}(x)}{\left|w_{k}(x)\right|} M & \text { if }\left|w_{k}(x)\right|>M,
\end{array} \text { for } x \in \Omega \text { and } k \in \mathbb{N} .\right.
$$

Since $u$ and $u_{0}$ are in $L_{M}^{\infty}\left(\Omega, \mathbb{R}^{N}\right)$ the functions $\tilde{w}_{k}$ are still in $\mathscr{D}$ and converge to $u$ in $L^{1}\left(\Omega, \mathbb{R}^{N}\right)$. Noting

$$
\left|\nabla \tilde{w}_{k}\right| \leqq\left|\nabla w_{k}\right|
$$

we moreover find that $D \tilde{w}_{k}$ converges to $D u$ weakly-* in the sense of measures on $\Omega$. Then using (5.4) again and invoking semicontinuity we find that $\left(\mathscr{L}^{n}, D \tilde{w}_{k}\right)$ converges strictly to $\left(\mathscr{L}^{n}, D u\right)$ in the sense of measures on $\Omega$. we deduce

By the continuity part of Theorem 2.4 (applied as in Remark 2.5; recall $u=u_{0}$ on $\partial \Omega$ )

$$
F\left[w_{k}\right]=\mathscr{F}^{\mathscr{D}}\left[w_{k}\right] \underset{k \rightarrow \infty}{\longrightarrow} \mathscr{F}^{\mathscr{D}}[u]=\inf _{\mathscr{D}} F
$$

where we used (1.9) for the last equality. Thus the sequence $\left(w_{k}\right)_{k \in \mathbb{N}}$ is minimizing for $F$ in $\mathscr{D}$. Replacing $\left(w_{k}\right)_{k \in \mathbb{N}}$ by a subsequence, if necessary, we assume that

$$
F\left[w_{k}\right] \leqq \inf _{\mathscr{D}} F+\frac{1}{8 k^{2}}
$$

holds for all $k \in \mathbb{N}$.

We now fix some number

$$
p>n,
$$


depending only on the dimension $n$. In order to work with a $W^{1, p}$-regularization we reduce to a minimizing sequence of class $W^{1, p}$ : First approximating the boundary values we choose a sequence ${ }^{15)}\left(u_{0 ; k}\right)_{k \in \mathbb{N}}$ in $W^{1, p}\left(\Omega, \mathbb{R}^{N}\right) \cap L_{M}^{\infty}\left(\Omega, \mathbb{R}^{N}\right)$ such that

$$
\left\|u_{0 ; k}-u_{0}\right\|_{W^{1,1}\left(\Omega, \mathbb{R}^{N}\right)} \leqq \frac{1}{8 L k^{2}}
$$

holds for all $k \in \mathbb{N}$, where $L$ is the constant from (5.1). Then we define

$$
\mathscr{D}_{k}:=\left(u_{0 ; k}+W_{0}^{1, p}\left(\Omega, \mathbb{R}^{N}\right)\right)
$$

and we record that $\mathscr{D}_{k} \subset W^{1, p}\left(\Omega, \mathbb{R}^{N}\right)$ holds by the above choice of $u_{0 ; k}$. Since $w_{k}-u_{0}$ is in $W_{0}^{1,1}\left(\Omega, \mathbb{R}^{N}\right) \cap L_{2 M}^{\infty}\left(\Omega, \mathbb{R}^{N}\right)$, we may find a function $v_{k} \in \mathscr{D}_{k} \cap L_{3 M}^{\infty}\left(\Omega, \mathbb{R}^{N}\right)$ with

$$
\left\|\left(v_{k}-u_{0 ; k}\right)-\left(w_{k}-u_{0}\right)\right\|_{W^{1,1}\left(\Omega, \mathbb{R}^{N}\right)} \leqq \frac{1}{8 L k^{2}}
$$

and consequently

$$
\left\|v_{k}-w_{k}\right\|_{W^{1,1}\left(\Omega, \mathbb{R}^{N}\right)} \leqq \frac{1}{4 L k^{2}} .
$$

Using (5.1) we get

$$
\inf _{\mathscr{D}} F=\inf _{u_{0}+W_{0}^{1, p}\left(\Omega, \mathbb{R}^{N}\right)} F \leqq \inf _{\mathscr{D} k} F+\frac{1}{8 k^{2}}
$$

and invoking (5.5) we come up with

$$
F\left[v_{k}\right] \leqq F\left[w_{k}\right]+\frac{1}{4 k^{2}} \leqq \inf _{\mathscr{D}} F+\frac{3}{8 k^{2}} \leqq \inf _{\mathscr{D}_{k}} F+\frac{1}{2 k^{2}} .
$$

We choose ${ }^{16)}$ a convex $C^{2}$-function $g: \mathbb{R}^{N} \rightarrow[0, \infty)$ such that for all $y \in \mathbb{R}^{N}$ we have

$$
g(y)=0 \text { whenever }|y| \leqq 1, \quad \exp \left(|y|^{4}\right) \leqq 3+g(y)
$$

In particular, $g$ grows exponentially. Next, we introduce the abbreviations

$$
\begin{aligned}
V_{k} & :=1+\int_{\Omega}\left(1+\left|\nabla v_{k}\right|^{2}\right)^{\frac{p}{2}} d x \\
f_{k}(z) & :=f(z)+\frac{1}{2 V_{k} k^{2}}\left(1+|z|^{2}\right)^{\frac{p}{2}},
\end{aligned}
$$

15) Such a sequence can be obtained, for instance, by mollifying an extension of $u_{0}$ to all of $\mathbb{R}^{n}$.

16) Such a function $g$ can be constructed by mollifying $\left[\exp \left(|y|^{4}\right)-A\right]_{+}$, where $\exp (1)<A<3$ is a parameter. 
and we notice that $(\mathrm{H} 2)$ and some computations give

$$
\begin{aligned}
{\left[\gamma(1+|z|)^{-3}+\frac{c}{V_{k} k^{2}}(1+|z|)^{p-2}\right]|\tilde{z}|^{2} } & \leqq \nabla^{2} f_{k}(z)(\tilde{z}, \tilde{z}) \\
& \leqq\left[\Gamma(1+|z|)^{-1}+\frac{C}{V_{k} k^{2}}(1+|z|)^{p-2}\right]|\tilde{z}|^{2}
\end{aligned}
$$

for all $z, \tilde{z} \in \mathbb{R}^{N n}$, with constants $c$ and $C$ depending only ${ }^{17)}$ on $n$ and $N$. Moreover, letting

$$
F_{k}[w]:= \begin{cases}\int_{\Omega} f_{k}(\nabla w) d x+\int_{\Omega} g\left(\frac{w}{3 M}\right) d x & \text { for } w \in \mathscr{D}_{k}, \\ \infty & \text { for } w \in W^{-1,1}\left(\Omega, \mathbb{R}^{N}\right) \backslash \mathscr{D}_{k}\end{cases}
$$

we define auxiliary functionals $F_{k}$. Noting $g\left(\frac{v_{k}}{3 M}\right) \equiv 0$, we have

$$
F_{k}\left[v_{k}\right] \leqq F\left[v_{k}\right]+\frac{1}{2 k^{2}} \leqq \inf _{\mathscr{D}_{k}} F+\frac{1}{k^{2}} \leqq \inf _{W^{-1,1}\left(\Omega, \mathbb{R}^{N}\right)} F_{k}+\frac{1}{k^{2}},
$$

and from Lemma 2.6 we deduce that $F_{k}$ is lower semicontinuous with respect to convergence in the norm of $W^{-1,1}\left(\Omega, \mathbb{R}^{N}\right)$; thus we may apply Ekeland's variational principle to each $F_{k}$ coming out with a sequence $\left(u_{k}\right)_{k \in \mathbb{N}}$ in $W^{-1,1}\left(\Omega, \mathbb{R}^{N}\right)$ such that

$$
\begin{gathered}
\left\|u_{k}-v_{k}\right\|_{W^{-1,1}\left(\Omega, \mathbb{R}^{N}\right)} \leqq \frac{1}{k} \\
F_{k}\left[u_{k}\right] \leqq F_{k}[w]+\frac{1}{k}\left\|w-u_{k}\right\|_{W^{-1,1}\left(\Omega, \mathbb{R}^{N}\right)} \quad \text { for all } w \in W^{-1,1}\left(\Omega, \mathbb{R}^{N}\right)
\end{gathered}
$$

In particular, we have

$$
\begin{aligned}
& \int_{\Omega}\left[\gamma\left|\nabla u_{k}\right|+\frac{1}{2 V_{k} k^{2}}\left(1+\left|\nabla u_{k}\right|^{2}\right)^{\frac{p}{2}}+g\left(\frac{u_{k}}{3 M}\right)\right] d x \\
& \quad \leqq F_{k}\left[u_{k}\right] \leqq F_{k}\left[v_{k}\right]+\frac{1}{k}\left\|v_{k}-u_{k}\right\|_{W^{-1,1}\left(\Omega, \mathbb{R}^{N}\right)}<\infty
\end{aligned}
$$

from which we infer $u_{k} \in \mathscr{D}_{k}$. To get another estimate for the left-hand side of the previous inequality we go through the above considerations, use the right-hand side of $(\mathrm{H} 1)$, and estimate

$$
F_{k}\left[v_{k}\right] \leqq \frac{C}{k^{2}}+\mathscr{F}^{\mathscr{D}}[u] \leqq C\left(\frac{1}{k^{2}}+\mathscr{L}^{n}(\Omega)+|D u|(\Omega)\right)
$$


with the result

$$
\begin{gathered}
\int_{\Omega}\left[\left|\nabla u_{k}\right|+\frac{1}{V_{k} k^{2}}\left(1+\left|\nabla u_{k}\right|^{2}\right)^{\frac{p}{2}}+\exp \left(\left|\frac{u_{k}}{3 M}\right|^{4}\right)\right] d x \\
\leqq C\left(\frac{1}{k^{2}}+\mathscr{L}^{n}(\Omega)+|D u|(\Omega)\right)
\end{gathered}
$$

for all $k \in \mathbb{N}$, where $C$ depends only on $\gamma$ and $\Gamma$. Now we return to the minimality property in (5.10). Using first-order criteria for minimality as for (4.4) we come up with the perturbed Euler equation:

$$
\left|\int_{\Omega} \nabla f_{k}\left(\nabla u_{k}\right) \cdot \nabla \varphi d x+\int_{\Omega} \nabla g\left(\frac{u_{k}}{3 M}\right) \cdot \frac{\varphi}{3 M} d x\right| \leqq \frac{1}{k}\|\varphi\|_{W^{-1,1}\left(\Omega, \mathbb{R}^{N}\right)}
$$

for all $\varphi \in W_{0}^{1, p}\left(\Omega, \mathbb{R}^{N}\right)$. We record that in view of (5.6) and Sobolev's embedding ${ }^{18)}$ we have

$$
u_{k} \in L^{\infty}\left(\Omega, \mathbb{R}^{N}\right)
$$

and thus here and in the following the integrals involving $g$ are finite.

5.2. Estimates for first and second derivatives. Next exploiting (5.12) we will derive some estimates for the functions $u_{k}$. In fact, we will first establish some estimates for the second derivatives, and then we will derive a uniform $L \log L$-bound for the first derivatives.

Let us start proving that the second derivatives exist and are square integrable. have

Lemma 5.1. For the sequence $\left(u_{k}\right)_{k \in \mathbb{N}}$ in $W^{1, p}\left(\Omega, \mathbb{R}^{N}\right)$, constructed in Section 5.1, we

$$
u_{k} \in W_{\mathrm{loc}}^{2,2}\left(\Omega, \mathbb{R}^{N}\right) \quad \text { and } \quad\left(1+\left|\nabla u_{k}\right|\right)^{p-2}\left|\nabla^{2} u_{k}\right|^{2} \in L_{\mathrm{loc}}^{1}\left(\Omega, \mathbb{R}^{N}\right)
$$

Proof. In this proof we establish estimates which are not uniform in $k$ and thus we allow that all our constants depend on $k$. For $s \in\{1,2,3, \ldots, n\}$ and $h \in \mathbb{R}$ we use the notation

$$
\Delta_{h}^{s} v(x):=\frac{v\left(x+h e_{s}\right)-v(x)}{h}
$$

for difference quotients, where $e_{s}$ denotes the $s$ th canonical basis vector in $\mathbb{R}^{n}$. Now we consider a nonnegative function $\eta \in C_{\mathrm{cpt}}^{\infty}(\Omega)$ and suppose $|h|<\operatorname{dist}(\operatorname{spt} \eta, \partial \Omega)$. Testing (5.12) with $\varphi=\Delta_{-h}^{s}\left(\eta^{2} \Delta_{h}^{s} u_{k}\right)$, using partial integration for difference quotients, and discarding the small factor $1 / k$ in $(5.12)$ we find

18) Most of the arguments in this section would work in a simpler way with the choice $p=2$. However, it is at this point that we are forced to take $p$ according to (5.6). 


$$
\begin{aligned}
& \int_{\Omega} \eta^{2} \Delta_{h}^{s}\left[\nabla f_{k}\left(\nabla u_{k}\right)\right] \cdot \Delta_{h}^{s} \nabla u_{k} d x+2 \int_{\Omega} \eta \Delta_{h}^{s}\left[\nabla f_{k}\left(\nabla u_{k}\right)\right] \cdot\left(\Delta_{h}^{s} u_{k} \otimes \nabla \eta\right) d x \\
& +\int_{\Omega} \eta^{2} \Delta_{h}^{s}\left[\nabla g\left(\frac{u_{k}}{3 M}\right)\right] \cdot \frac{\Delta_{h}^{s} u_{k}}{3 M} d x \\
& \leqq\left\|\Delta_{-h}^{s}\left(\eta^{2} \Delta_{h}^{s} u_{k}\right)\right\|_{W^{-1,1}\left(\Omega, \mathbb{R}^{N}\right)} \\
& \leqq \int_{\Omega}\left|\Delta_{-h}^{s}\left(\eta^{2} \Delta_{h}^{s} u_{k}\right)\right| d x \\
& \leqq \int_{\Omega}\left|\partial^{s}\left(\eta^{2} \Delta_{h}^{s} u_{k}\right)\right| d x \\
& \leqq \int_{\Omega} \eta^{2}\left|\Delta_{h}^{s} \nabla u_{k}\right| d x+2 \int_{\Omega} \eta\left|\Delta_{h}^{s} u_{k} \otimes \nabla \eta\right| d x
\end{aligned}
$$

Here we also used (2.2) and a standard estimate for difference quotients. By the convexity of $g$ we get

$$
\begin{aligned}
& \Delta_{h}^{s}\left[\nabla g\left(\frac{u_{k}}{3 M}\right)\right](x) \cdot \frac{\Delta_{h}^{s} u_{k}(x)}{3 M} \\
& \quad=\int_{0}^{1} \nabla^{2} g\left(\frac{(1-t) u_{k}(x)+t u_{k}\left(x+h e_{s}\right)}{3 M}\right) d t\left(\frac{\Delta_{h}^{s} u_{k}(x)}{3 M}, \frac{\Delta_{h}^{s} u_{k}(x)}{3 M}\right) \geqq 0 .
\end{aligned}
$$

Now we introduce for every $x \in \operatorname{spt} \eta$ the positive symmetric bilinear form

$$
\mathscr{A}_{k}^{x}:=\int_{0}^{1} \nabla^{2} f_{k}\left((1-t) \nabla u_{k}(x)+t \nabla u_{k}\left(x+h e_{s}\right)\right) d t .
$$

We infer

$$
\begin{aligned}
& \int_{\Omega} \eta^{2} \mathscr{A}_{k}^{\bullet}\left(\Delta_{h}^{s} \nabla u_{k}, \Delta_{h}^{s} \nabla u_{k}\right) d x \\
& \quad \leqq-2 \int_{\Omega} \eta \mathscr{A}_{k}^{\bullet}\left(\Delta_{h}^{s} \nabla u_{k}, \Delta_{h}^{s} u_{k} \otimes \nabla \eta\right) d x+\int_{\Omega} \eta^{2}\left|\Delta_{h}^{s} \nabla u_{k}\right| d x+2 \int_{\Omega} \eta\left|\Delta_{h}^{s} u_{k} \otimes \nabla \eta\right| d x
\end{aligned}
$$

Using Young's inequality for the positive forms $\mathscr{A}_{k}^{\bullet}$ and absorbing a term on the left-hand side we get

$$
\begin{aligned}
\int_{\Omega} \eta^{2} \mathscr{A}_{k}^{\bullet}\left(\Delta_{h}^{s} \nabla u_{k}, \Delta_{h}^{s} \nabla u_{k}\right) d x \leqq & 4 \int_{\Omega} \mathscr{A}_{k}^{\bullet}\left(\Delta_{h}^{s} u_{k} \otimes \nabla \eta, \Delta_{h}^{s} u_{k} \otimes \nabla \eta\right) d x \\
& +2 \int_{\Omega} \eta^{2}\left|\Delta_{h}^{s} \nabla u_{k}\right| d x+4 \int_{\Omega} \eta\left|\Delta_{h}^{s} u_{k} \otimes \nabla \eta\right| d x
\end{aligned}
$$

Now we recall from [41], Lemma 2.1, that for all $z_{1}, z_{2} \in \mathbb{R}^{N n}$ there holds

$$
\int_{0}^{1}\left(1+\left|(1-t) z_{1}+t z_{2}\right|\right)^{p-2} d t \geqq c\left(1+\left|z_{1}\right|+\left|z_{2}\right|\right)^{p-2} .
$$


Using this in (5.8) we have in particular

$$
\begin{aligned}
& \frac{c}{V_{k} k^{2}}\left(1+\left|\nabla u_{k}(x)\right|\right)^{p-2}|\xi|^{2} \\
& \quad \leqq \mathscr{A}_{k}^{x}(\xi, \xi) \leqq\left(\Gamma+\frac{C}{V_{k} k^{2}}\left(1+\left|\nabla u_{k}(x)\right|+\left|\nabla u_{k}\left(x+h e_{s}\right)\right|\right)^{p-2}\right)|\xi|^{2}
\end{aligned}
$$

with constants depending only on $n$ and $N$. With these estimates for $\mathscr{A}_{k}^{\bullet}$ we find

$$
\begin{aligned}
& \int_{\Omega} \eta^{2}\left(1+\left|\nabla u_{k}\right|\right)^{p-2}\left|\Delta_{h}^{s} \nabla u_{k}\right|^{2} d x \\
& \leqq C\left[\int_{\Omega}\left(1+\left|\nabla u_{k}(x)\right|+\left|\nabla u_{k}\left(x+h e_{s}\right)\right|\right)^{p-2}|\nabla \eta|^{2}\left|\Delta_{h}^{s} u_{k}\right|^{2} d x\right. \\
& \left.+\int_{\Omega} \eta^{2}\left|\Delta_{h}^{s} \nabla u_{k}\right| d x+\int_{\Omega} \eta|\nabla \eta|\left|\Delta_{h}^{s} u_{k}\right| d x\right]
\end{aligned}
$$

for a constant $C$ depending only on $n, N, \Gamma, k$, and $V_{k}$. Employing Young's inequality and absorbing again we arrive at

$$
\begin{aligned}
& \int_{\Omega} \eta^{2}\left(1+\left|\nabla u_{k}\right|\right)^{p-2}\left|\Delta_{h}^{s} \nabla u_{k}\right|^{2} d x \\
& \quad \leqq C \sup _{\Omega}\left(\eta^{2}+|\nabla \eta|^{2}\right) \int_{\Omega}\left(1+\left|\Delta_{h}^{s} u_{k}\right|^{2}+\left|\Delta_{h}^{s} u_{k}\right|^{p}+\left|\nabla u_{k}\right|^{p}\right) d x .
\end{aligned}
$$

Letting $h \rightarrow 0$ and exploiting $u_{k} \in W^{1, p}\left(\Omega, \mathbb{R}^{N}\right)$ we deduce the first claim, that is $u_{k} \in W_{\text {loc }}^{2,2}\left(\Omega, \mathbb{R}^{N}\right)$. Moreover, $\Delta_{h}^{s} \nabla u_{k}$ converges strongly in $L_{\text {loc }}^{2}\left(\Omega, \mathbb{R}^{N n}\right)$ to $\partial^{s} \nabla u_{k}$, and the second claim follows via Fatou's lemma.

Next, as in [15], Lemma 3.2, we derive uniform estimates involving the second derivatives $\nabla^{2} u_{k}$.

Lemma 5.2. For the sequence $\left(u_{k}\right)_{k \in \mathbb{N}}$ in $W^{1, p}\left(\Omega, \mathbb{R}^{N}\right)$, constructed in Section 5.1, there holds

$$
\begin{array}{r}
\int_{\Omega} \eta^{2}\left[\frac{\left|\nabla^{2} u_{k}\right|^{2}}{\left(1+\left|\nabla u_{k}\right|\right)^{3}}+\frac{1}{V_{k} k^{2}}\left(1+\left|\nabla u_{k}\right|\right)^{p-2}\left|\nabla^{2} u_{k}\right|^{2}\right] d x \\
\leqq C \sup _{\Omega}\left(\eta^{2} / k+|\nabla \eta|^{2}\right)\left(\frac{1}{k^{2}}+\mathscr{L}^{n}(\Omega)+|D u|(\Omega)\right)
\end{array}
$$

where $\eta \in C_{\mathrm{cpt}}^{\infty}(\Omega)$ is a nonnegative function, and $C$ depends only on $n, N, \gamma, \Gamma$, but not on $k$.

Proof. We first record that (5.8) yields in particular

$$
\begin{array}{r}
\left|\nabla^{2} f_{k}(z)\right| \leqq C(1+|z|)^{p-2}, \\
\text { Bereitgestellt von | Universitaetsbibliothek Augsburg } \\
\text { Angemeldet } \\
\text { Heruntergeladen am | 04.02.19 08:18 }
\end{array}
$$


where $C$ depends only on $n, N, \Gamma, k$, and $V_{k}$. From Lemma 5.1 we deduce

$$
\left(1+\left|\nabla u_{k}\right|\right)^{p-2}\left|\nabla^{2} u_{k}\right| \in L_{\text {loc }}^{\frac{p}{p-1}}(\Omega)
$$

via Hölder's inequality, and by the chain rule we get

$$
\partial^{s}\left[\nabla f_{k}\left(\nabla u_{k}\right)\right]=\nabla^{2} f_{k}\left(\nabla u_{k}\right)\left(\partial^{s} \nabla u_{k}, \cdot\right) \in L_{\text {loc }}^{\frac{p}{p-1}}\left(\Omega,\left(\mathbb{R}^{N n}\right)^{*}\right)
$$

for $s \in\{1,2, \ldots, n\}$. Keeping (5.13) in mind, we also have

$$
\partial^{s}\left[\nabla g\left(\frac{u_{k}}{3 M}\right)\right]=\nabla^{2} g\left(\frac{u_{k}}{3 M}\right)\left(\frac{\partial^{s} u_{k}}{3 M}, \cdot\right) \in L_{\mathrm{loc}}^{p}\left(\Omega,\left(\mathbb{R}^{N}\right)^{*}\right)
$$

For $\psi \in C_{\mathrm{cpt}}^{\infty}\left(\Omega, \mathbb{R}^{N}\right)$ we next use $\varphi=-\partial^{s} \psi$ as a test function in (5.12). Using also (2.3) we get

$$
\begin{gathered}
\int_{\Omega} \nabla^{2} f_{k}\left(\nabla u_{k}\right)\left(\partial^{s} \nabla u_{k}, \nabla \psi\right) d x+\int_{\Omega} \nabla^{2} g\left(\frac{u_{k}}{3 M}\right)\left(\frac{\partial^{s} u_{k}}{3 M}, \frac{\psi}{3 M}\right) d x \\
\leqq \frac{1}{k}\left\|\partial^{s} \psi\right\|_{W^{-1,1}\left(\Omega, \mathbb{R}^{N}\right)} \leqq \frac{1}{k} \int_{\Omega}|\psi| d x
\end{gathered}
$$

Taking into account the above integrability properties, a standard approximation argument shows that (5.17) holds in fact for every $\psi \in W_{\mathrm{cpt}}^{1, p}\left(\Omega, \mathbb{R}^{N}\right)$. Next we reason that the inequality is still valid for $\psi:=\eta^{2} \partial^{s} u_{k}$, even though this function need not be in $W_{\mathrm{cpt}}^{1, p}\left(\Omega, \mathbb{R}^{N}\right)$. To this end we first plug in $\psi_{h}:=\eta^{2} \Delta_{h}^{s} u_{k} \in W_{\mathrm{cpt}}^{1, p}\left(\Omega, \mathbb{R}^{N}\right)$ with small $|h|$. Then $\psi_{h}$ converges to $\psi$ strongly in $L^{p}\left(\Omega, \mathbb{R}^{N}\right)$ as $h \rightarrow 0$, and furthermore going back to the last formula in the proof of Lemma 5.1 we infer that $\nabla \psi_{h}$ remains bounded in the weighted Lebesgue space $\widetilde{L^{2}}:=L^{2}\left(\Omega, \mathbb{R}^{N n} ;\left(1+\left|\nabla u_{k}\right|\right)^{p-2} \cdot \mathscr{L}^{n}\right)$. It follows that $\nabla \psi_{h}$ converges weakly to $\nabla \psi$ in $\widetilde{L^{2}}$. By (5.16), Hölder's inequality, and Lemma 5.1, the mapping

$$
\psi \mapsto \int_{\Omega} \nabla^{2} f_{k}\left(\nabla u_{k}\right)\left(\partial^{s} \nabla u_{k}, \nabla \psi\right) d x
$$

defines a continuous linear form on $\widetilde{L^{2}}$. Hence passing to the limit $h \rightarrow 0$ and exploiting the above convergences we find that (5.17) still holds for $\psi=\eta^{2} \partial^{s} u_{k}$ as claimed. Now we repeat the arguments from the proof of Lemma 5.1, just in terms of derivatives rather than difference quotients. Proceeding in this way up to (5.14) (and keeping the factor $1 / k$ this time) we come out with

$$
\begin{aligned}
& \int_{\Omega} \eta^{2} \nabla^{2} f_{k}\left(\nabla u_{k}\right)\left(\partial^{s} \nabla u_{k}, \partial^{s} \nabla u_{k}\right) d x \\
& \quad \leqq 4 \int_{\Omega} \nabla^{2} f_{k}\left(\nabla u_{k}\right)\left(\partial^{s} u_{k} \otimes \nabla \eta, \partial^{s} u_{k} \otimes \nabla \eta\right) d x+\frac{2}{k} \int_{\Omega} \eta^{2}\left|\partial^{s} u_{k}\right| d x
\end{aligned}
$$

Invoking the estimates for $\nabla^{2} f_{k}$ in (5.8) and summing over $s$ yields 


$$
\begin{aligned}
& \int_{\Omega} \eta^{2}\left[\frac{\left|\nabla^{2} u_{k}\right|^{2}}{\left(1+\left|\nabla u_{k}\right|\right)^{3}}+\frac{1}{V_{k} k^{2}}\left(1+\left|\nabla u_{k}\right|\right)^{p-2}\left|\nabla^{2} u_{k}\right|^{2}\right] d x \\
& \quad \leqq C \sup _{\Omega}\left(\eta^{2} / k+|\nabla \eta|^{2}\right) \int_{\Omega}\left(\left|\nabla u_{k}\right|+\frac{1}{V_{k} k^{2}}\left(1+\left|\nabla u_{k}\right|\right)^{p}\right) d x .
\end{aligned}
$$

Taking into account (5.11) we arrive at the claim.

We next adapt the proof of [15], Theorem 4.1, to our situation and derive a uniform $L \log L$-estimate for the gradients $\nabla u_{k}$. To this end we test (5.12) once more and we employ both the uniform bounds given by (5.11) and the estimate from Lemma 5.2.

Lemma 5.3. For the sequence $\left(u_{k}\right)_{k \in \mathbb{N}}$ in $W^{1, p}\left(\Omega, \mathbb{R}^{N}\right)$, constructed in Section 5.1, and every ball $B_{2 r}\left(x_{0}\right) \subset \Omega$ we have

$$
\begin{aligned}
& \int_{B_{r}\left(x_{0}\right)}\left|\nabla u_{k}\right| \log \left(1+\left|\nabla u_{k}\right|^{2}\right) d x \\
& \quad \leqq C\left(\lambda+\frac{M+M r+M^{2}}{k}+\frac{M}{r}+\frac{M^{2}}{r^{2}}\right)\left(\frac{1}{k^{2}}+\mathscr{L}^{n}(\Omega)+|D u|(\Omega)\right)
\end{aligned}
$$

with a constant $C$ depending only on $n, N, \gamma$, and $\Gamma$, and in particular independent of $k$. Here, $M$ was defined in (5.2) as $\sup |u|$.

Proof. We will use the following estimates, which are available by Lemma 2.7, Lemma 2.8, and the properties of $g$ :

$$
\begin{gathered}
|\nabla f(z)| \leqq C, \\
\nabla f(z) \cdot z \geqq \gamma|z|-\lambda, \\
\nabla g(y) \cdot y \geqq 0 .
\end{gathered}
$$

Now we consider a cut-off function $\eta \in C_{\text {cpt }}^{\infty}(\Omega)$ satisfying $\mathbb{1}_{B_{r}\left(x_{0}\right)} \leqq \eta \leqq \mathbb{1}_{B_{2 r}\left(x_{0}\right)}$ and $|\nabla \eta| \leqq \frac{2}{r}$ on $\Omega$. Then we define

$$
\varphi:=\eta^{2} u_{k} \log \left(1+\left|\nabla u_{k}\right|^{2}\right)
$$

and compute for every $s \in\{1,2, \ldots, n\}$

$$
\begin{aligned}
\partial^{s} \varphi= & 2 \eta\left(\partial^{s} \eta\right) u_{k} \log \left(1+\left|\nabla u_{k}\right|^{2}\right)+\eta^{2}\left(\partial^{s} u_{k}\right) \log \left(1+\left|\nabla u_{k}\right|^{2}\right) \\
& +2 \eta^{2} u_{k} \frac{\nabla u_{k} \cdot \partial^{s} \nabla u_{k}}{1+\left|\nabla u_{k}\right|^{2}}
\end{aligned}
$$

Since $\varphi$ is not immediately admissible in (5.12), we first plug in the approximations $\varphi_{h}:=\eta^{2} u_{k} \log \left(1+\sum_{i=1}^{n}\left|\Delta_{h}^{i} u_{k}\right|^{2}\right)$. In view of (5.13), $\varphi_{h}$ converges to $\varphi$ in $L^{p}\left(\Omega, \mathbb{R}^{N}\right)$ and 
moreover one finds that $\nabla \varphi_{h}$ remains bounded ${ }^{19)}$ in $\widetilde{L^{2}}:=L^{2}\left(\Omega, \mathbb{R}^{N n} ;\left(1+\left|\nabla u_{k}\right|\right)^{p-2} \cdot \mathscr{L}^{n}\right)$ for $h \rightarrow 0$. Consequently, arguing as in the proof of Lemma 5.2 we may test (5.12) with $\varphi$. Taking into account (5.21) and (2.2) we infer

$$
\int_{\Omega} \nabla f_{k}\left(\nabla u_{k}\right) \cdot \nabla \varphi d x \leqq \frac{1}{k} \int_{\Omega}|\varphi| d x .
$$

Now we apply the above formula for $\partial^{s} \varphi$ on the left-hand side of the last inequality. Then we shift all the terms containing $u_{k}$ itself to the right-hand side. We get

$$
\begin{aligned}
& \int_{\Omega} \eta^{2} \nabla f_{k}\left(\nabla u_{k}\right) \cdot \nabla u_{k} \log \left(1+\left|\nabla u_{k}\right|^{2}\right) d x \\
& \leqq \int_{\Omega}\left|\nabla f_{k}\left(\nabla u_{k}\right)\right|\left|u_{k}\right|\left(2 \eta|\nabla \eta| \log \left(1+\left|\nabla u_{k}\right|^{2}\right)+2 \eta^{2} \frac{\left|\nabla u_{k}\right|\left|\nabla^{2} u_{k}\right|}{1+\left|\nabla u_{k}\right|^{2}}\right) d x \\
& \quad+\frac{1}{k} \int_{\Omega} \eta^{2}\left|u_{k}\right| \log \left(1+\left|\nabla u_{k}\right|^{2}\right) d x .
\end{aligned}
$$

In the next step we recall $\nabla f_{k}(z)=\nabla f(z)+\frac{p}{2 V_{k} k^{2}}\left(1+|z|^{2}\right)^{\frac{p-2}{2}} z$ and use (5.19) on the right-hand side of the previous inequality. Moreover, we estimate $\log \left(1+t^{2}\right) \leqq 4 \sqrt{t}$ and $\eta|\nabla \eta| \leqq 2 / r$ coming out with

$$
\begin{aligned}
& \int_{\Omega} \eta^{2} \nabla f_{k}\left(\nabla u_{k}\right) \cdot \nabla u_{k} \log \left(1+\left|\nabla u_{k}\right|^{2}\right) d x \\
& \leqq C\left[\left(\frac{1}{k}+\frac{1}{r}\right) \int_{\Omega}\left|u_{k}\right|\left(\left|\nabla u_{k}\right|^{\frac{1}{2}}+\frac{1}{V_{k} k^{2}}\left(1+\left|\nabla u_{k}\right|\right)^{p-\frac{1}{2}}\right) d x\right. \\
& \left.\quad+\int_{\Omega} \eta^{2}\left|u_{k}\right| \frac{\left|\nabla^{2} u_{k}\right|}{1+\left|\nabla u_{k}\right|} d x+\int_{\Omega} \frac{\eta^{2}\left|u_{k}\right|}{V_{k} k^{2}}\left(1+\left|\nabla u_{k}\right|\right)^{p-2}\left|\nabla^{2} u_{k}\right| d x\right] \\
& =: C\left[I_{k}+I I_{k}+I I I_{k}\right] .
\end{aligned}
$$

To control $I_{k}$ we use Young's inequality, $\frac{1}{V_{k} k^{2}} \leqq 1$, and (5.11) as follows:

$$
\begin{aligned}
I_{k} & \leqq 3\left(\frac{M}{k}+\frac{M}{r}\right) \int_{\Omega}\left[\left|\frac{u_{k}}{3 M}\right|^{2}+\left|\frac{u_{k}}{3 M}\right|^{2 p}+\left|\nabla u_{k}\right|+\frac{1}{V_{k} k^{2}}\left(1+\left|\nabla u_{k}\right|\right)^{p}\right] d x \\
& \leqq C\left(\frac{M}{k}+\frac{M}{r}\right) \int_{\Omega}\left[\exp \left(\left|\frac{u_{k}}{3 M}\right|^{4}\right)+\left|\nabla u_{k}\right|+\frac{1}{V_{k} k^{2}}\left(1+\left|\nabla u_{k}\right|\right)^{p}\right] d x \\
& \leqq C\left(\frac{M}{k}+\frac{M}{r}\right)\left(\frac{1}{k^{2}}+\mathscr{L}^{n}(\Omega)+|D u|(\Omega)\right)
\end{aligned}
$$

The term $I I I_{k}$ in (5.23) is estimated similarly, but additionally exploits (5.15):

19) Evidently, $\partial^{s} \varphi_{h}$ is given by a formula analogous to (5.22). To control the second term on the right-hand side of this formula in $\widetilde{L^{2}}$ we use the fact that $\nabla u_{k} \in L_{\text {loc }}^{q}\left(\Omega, \mathbb{R}^{N n}\right)$ holds for some $q>p$. The latter integrability follows in turn from Lemma 5.1 by the chain rule and Sobolev's embedding. 


$$
\begin{aligned}
I I I_{k} \leqq & 3 M r \int_{\Omega} \frac{\eta^{2}}{V_{k} k^{2}}\left(1+\left|\nabla u_{k}\right|\right)^{p-2}\left|\nabla^{2} u_{k}\right|^{2} d x \\
& +\frac{3 M}{r} \int_{\Omega}\left[\left|\frac{u_{k}}{3 M}\right|^{p}+\frac{1}{V_{k} k^{2}}\left(1+\left|\nabla u_{k}\right|\right)^{p}\right] d x \\
\leqq & C\left(\frac{M r}{k}+\frac{M}{r}\right)\left(\frac{1}{k^{2}}+\mathscr{L}^{n}(\Omega)+|D u|(\Omega)\right) .
\end{aligned}
$$

Finally, we treat $I I_{k}$ via the Orlicz-Young inequality from Lemma 2.12, Young's inequality, (5.15), and (5.11). We have

$$
\begin{aligned}
I I_{k}= & 3 M \int_{\Omega} \eta^{2} \frac{\left|\nabla^{2} u_{k}\right|}{\left(1+\left|\nabla u_{k}\right|\right)^{3 / 2}} \sqrt{\left|\frac{u_{k}}{3 M}\right|^{2}\left(1+\left|\nabla u_{k}\right|\right)} d x \\
\leqq & 3 M \int_{\Omega} \eta^{2} \frac{\left|\nabla^{2} u_{k}\right|}{\left(1+\left|\nabla u_{k}\right|\right)^{3 / 2}} \sqrt{\exp \left(\left|\frac{u_{k}}{3 M}\right|^{2}\right)+\left|\nabla u_{k}\right| \log \left(1+\left|\nabla u_{k}\right|^{2}\right)} d x \\
\leqq & 9\left(M r+M^{2} \varepsilon^{-1}\right) \int_{\Omega} \eta^{2} \frac{\left|\nabla^{2} u_{k}\right|^{2}}{\left(1+\left|\nabla u_{k}\right|\right)^{3}} d x+\frac{M}{r} \int_{\Omega} \exp \left(\left|\frac{u_{k}}{3 M}\right|^{2}\right) d x \\
& +\varepsilon \int_{\Omega} \eta^{2}\left|\nabla u_{k}\right| \log \left(1+\left|\nabla u_{k}\right|^{2}\right) d x \\
\leqq & C\left(\frac{M r+M^{2} \varepsilon^{-1}}{k}+\frac{M}{r}+\frac{M^{2} \varepsilon^{-1}}{r^{2}}\right)\left(\frac{1}{k^{2}}+\mathscr{L}^{n}(\Omega)+|D u|(\Omega)\right) \\
& +\varepsilon \int_{\Omega} \eta^{2}\left|\nabla u_{k}\right| \log \left(1+\left|\nabla u_{k}\right|^{2}\right) d x
\end{aligned}
$$

for all $\varepsilon>0$. Now we collect the estimates for the right-hand side of (5.23). We come out with

$$
\begin{aligned}
& \int_{\Omega} \eta^{2} \nabla f_{k}\left(\nabla u_{k}\right) \cdot \nabla u_{k} \log \left(1+\left|\nabla u_{k}\right|^{2}\right) d x \\
& \leqq \\
& \quad C\left(\frac{M+M r+M^{2} \varepsilon^{-1}}{k}+\frac{M}{r}+\frac{M^{2} \varepsilon^{-1}}{r^{2}}\right)\left(\frac{1}{k^{2}}+\mathscr{L}^{n}(\Omega)+|D u|(\Omega)\right) \\
& \quad+C \varepsilon \int_{\Omega} \eta^{2}\left|\nabla u_{k}\right| \log \left(1+\left|\nabla u_{k}\right|^{2}\right) d x .
\end{aligned}
$$

Next we deal with the left-hand side of (5.24). Recalling the definition of $f_{k}$ and (5.20) we have $\nabla f_{k}(z) \cdot z \geqq \gamma|z|-\lambda$. Using this with $\log \left(1+t^{2}\right) \leqq 2 t$ and once more (5.11) we find

$$
\begin{aligned}
& \int_{\Omega} \eta^{2} \nabla f_{k}\left(\nabla u_{k}\right) \cdot \nabla u_{k} \log \left(1+\left|\nabla u_{k}\right|^{2}\right) d x \\
& \quad \geqq \gamma \int_{\Omega} \eta^{2}\left|\nabla u_{k}\right| \log \left(1+\left|\nabla u_{k}\right|^{2}\right) d x-\lambda \int_{\Omega} \log \left(1+\left|\nabla u_{k}\right|^{2}\right) d x \\
& \quad \geqq \gamma \int_{\Omega} \eta^{2}\left|\nabla u_{k}\right| \log \left(1+\left|\nabla u_{k}\right|^{2}\right) d x-C \lambda\left(\frac{1}{k^{2}}+\mathscr{L}^{n}(\Omega)+|D u|(\Omega)\right) .
\end{aligned}
$$


At this point we fix $\varepsilon$ such that $C \varepsilon \leqq \gamma / 2$ holds for the constant $C$ in (5.24). Combining the previous estimate with (5.24) we arrive at

$$
\begin{aligned}
& \frac{\gamma}{2} \int_{\Omega} \eta^{2}\left|\nabla u_{k}\right| \log \left(1+\left|\nabla u_{k}\right|^{2}\right) d x \\
& \quad \leqq C\left(\lambda+\frac{M+M r+M^{2}}{k}+\frac{M}{r}+\frac{M^{2}}{r^{2}}\right)\left(\frac{1}{k^{2}}+\mathscr{L}^{n}(\Omega)+|D u|(\Omega)\right),
\end{aligned}
$$

and the claim follows by the choice of $\eta$.

5.3. Proofs of the uniqueness results. In this subsection we will prove Theorem 1.10 and Corollary 1.13 .

Proof of Theorem 1.10. Regularity. To prove the regularity results we continue working with the generalized minimizer $u$ and the minimizing sequence $\left(u_{k}\right)_{k \in \mathbb{N}}$ from Section 5.1, which were investigated in Section 5.2. By (5.3), (5.7), and (5.9) we have

$$
u_{k} \underset{k \rightarrow \infty}{\longrightarrow} u \text { in the norm of } W^{-1,1}\left(\Omega, \mathbb{R}^{N}\right) .
$$

However, from $(5.11)$ we deduce that a subsequence of $\left(u_{k}\right)_{k \in \mathbb{N}}$ converges weakly-* in $B V\left(\Omega, \mathbb{R}^{N}\right)$, and (5.25) is only needed to identify $u$ as the limit. In particular,

$$
\nabla u_{k} \cdot \mathscr{L}^{n} \text { converges weakly-* to } D u \text { in the sense of measures on } \Omega \text {. }
$$

Now we introduce the convex function $\Phi(z):=|z| \log \left(1+|z|^{2}\right)$. In view of the above convergence we may apply the semicontinuity part of Theorem 2.4 as in Remark 2.5, but with $\Phi$ in place of $f$, to deduce

$$
\int_{B_{r}\left(x_{0}\right)} \Phi(\nabla u) d x+\int_{B_{r}\left(x_{0}\right)} \Phi^{\infty}\left(\frac{d D^{s} u}{d\left|D^{s} u\right|}\right) d\left|D^{s} u\right| \leqq \liminf _{k \rightarrow \infty} \int_{B_{r}\left(x_{0}\right)} \Phi\left(\nabla u_{k}\right) d x
$$

By Lemma 5.3 we arrive at

$$
\begin{gathered}
\int_{B_{r}\left(x_{0}\right)} \Phi(\nabla u) d x+\int_{B_{r}\left(x_{0}\right)} \Phi^{\infty}\left(\frac{d D^{s} u}{d\left|D^{s} u\right|}\right) d\left|D^{s} u\right| \\
\leqq C\left(\lambda+\frac{M}{r}+\frac{M^{2}}{r^{2}}\right)\left(\mathscr{L}^{n}(\Omega)+|D u|(\Omega)\right) .
\end{gathered}
$$

In particular, the right-hand side and thus also the left-hand side of the last estimate is finite. Since $\Phi^{\infty}(z)=\infty$ holds for $z \neq 0$ and $B_{2 r}\left(x_{0}\right)$ is an arbitrary ball in $\Omega$, this means that $D^{s} u$ vanishes, $u \in W^{1,1}\left(\Omega, \mathbb{R}^{N}\right)$ holds, and moreover we have $\Phi(\nabla u) \in L_{\text {loc }}^{1}(\Omega)$.

$L \log L$-estimate. We recall from the beginning of Section 5 that $u$ minimizes with respect to its own boundary values. From this observation it follows that $u$ still minimizes on any ball $B_{2 r}\left(x_{0}\right) \subset \Omega$ and thus (5.26) still holds if we replace $\Omega$ by $B_{2 r}\left(x_{0}\right)$ (and $M$ by sup $|u|)$ on the right-hand side. Since we have already argued that $D^{s} u$ vanishes, this yields $B_{2 r}\left(x_{0}\right)$ 
the estimate of Theorem 1.10 - apart from the quadratic occurrence of sup $|u|$. To establish the precise form of the claim we finally reason that the term $M^{2} / r^{2}$ in (5.26) can be removed. Since this refinement is of secondary importance, we just outline how it is achieved:

We revisit the arguments of this section, and exploiting that the $W^{1,1}$-regularity of $u$ and a uniform $L \log L$-bound for $\nabla u_{k}$ are already available, we now modify the treatment of the term $I I_{k}$ on the right-hand side of (5.23) as follows. We obtain

$$
I I_{k} \leqq M r \int_{\Omega} \eta^{2} \frac{\left|\nabla^{2} u_{k}\right|^{2}}{\left(1+\left|\nabla u_{k}\right|\right)^{3}} d x+\frac{1}{M r} \int_{\Omega} \eta^{2}\left|u_{k}\right|^{2}\left(1+\left|\nabla u_{k}\right|\right) d x=: M r J_{k}+\frac{1}{M r} J J_{k}
$$

by Young's inequality, and $J_{k}$ is handled via $(5.15)$ as before. In order to deal with $J J_{k}$ we make use of Theorem B.2. Actually, it is not difficult to verify the assumptions of Theorem B.2 (i) for the sequence $\left(u_{k}\right)_{k \in \mathbb{N}}$ (but we skip the details here), and thus strong convergence $u_{k} \rightarrow u$ in $W^{1,1}\left(\Omega, \mathbb{R}^{N}\right)$ follows. For a suitable subsequence we infer $\left(u_{k_{l}}, \nabla u_{k_{l}}\right) \rightarrow(u, \nabla u)$ a.e. on $\Omega$, and moreover we estimate via Young's inequality and Lemma 2.12

$$
\begin{aligned}
& \int_{\mathrm{spt} \eta}\left|u_{k}\right|^{2}\left|\nabla u_{k}\right| \sqrt{\log \left(1+\left|u_{k}\right|^{4}\left|\nabla u_{k}\right|^{2}\right)} d x \\
& \leqq \int_{\mathrm{spt} \eta}\left|u_{k}\right|^{2}\left|\nabla u_{k}\right| \sqrt{\log \left(1+\left|\nabla u_{k}\right|^{2}\right)} d x+\int_{\mathrm{spt} \eta}\left|u_{k}\right|^{2}\left|\nabla u_{k}\right| \sqrt{\log \left(1+\left|u_{k}\right|^{4}\right)} d x \\
& \leqq \int_{\mathrm{spt} \eta}\left|\nabla u_{k}\right| \log \left(1+\left|\nabla u_{k}\right|^{2}\right) d x+C \int_{\mathrm{spt} \eta}\left|u_{k}\right|^{4}\left|\nabla u_{k}\right| d x \\
& \leqq C\left(1+M^{4}\right)\left[\int_{\mathrm{spt} \eta}\left|\nabla u_{k}\right| \log \left(1+\left|\nabla u_{k}\right|^{2}\right) d x+\int_{\Omega} \exp \left(\left|\frac{u_{k}}{3 M}\right|^{4}\right) d x\right] .
\end{aligned}
$$

In view of Lemma 5.3 and (5.11) the right-hand side of the last estimate remains bounded as $k \rightarrow \infty$ and hence the sequence $\left(\eta^{2}\left|u_{k}\right|^{2}\left(1+\left|\nabla u_{k}\right|\right)\right)_{k \in \mathbb{N}}$ is uniformly integrable on $\Omega$. By Vitali's convergence theorem we conclude

$$
\liminf _{k \rightarrow \infty} J J_{k} \leqq \lim _{l \rightarrow \infty} J J_{k_{l}}=\int_{\Omega} \eta^{2}|u|^{2}(1+|\nabla u|) d x \leqq M^{2}\left(\mathscr{L}^{n}(\Omega)+|D u|(\Omega)\right)
$$

and the refined version of (5.26) follows.

Uniqueness. Let us consider two bounded generalized minimizers $u$ and $v$ for $F$ in an arbitrary Dirichlet class $\mathscr{D}$. If $\nabla u \neq \nabla v$ holds on a set of positive measure, then the strict convexity of $f$ and the convexity of $f^{\infty}$ give the following contradiction:

$$
\mathscr{F}^{\mathscr{D}}\left[\frac{u+v}{2}\right]<\frac{1}{2}\left(\mathscr{F}^{\mathscr{D}}[u]+\mathscr{F}^{\mathscr{D}}[v]\right)=\min _{B V\left(\Omega, \mathbb{R}^{N}\right)} \mathscr{F}^{\mathscr{D}} .
$$

Thus we have $\nabla u=\nabla v$. Since we have already proved that $D^{s} u$ and $D^{s} v$ vanish, this means $D u=D v$ and since $\Omega$ is connected, the claim $u=v+y$ follows by the constancy theorem. 
Proof of Corollary 1.13. The claims follow ${ }^{20)}$ from Theorem 1.10 and Theorem 1.11, and in fact there are only a few points which need to be addressed:

Regularity. Concerning regularity the relevant point is that Theorem 1.11 just provides interior $L_{\text {loc }}^{\infty}$-regularity while Theorem 1.10 assumes global boundedness. However, by the same simple reasoning as for the $L \log L$-estimate in the proof of Theorem 1.10 we know that generalized minimizers also minimize on subdomains with respect to their own boundary values. Thus we may still apply Theorem 1.10 on subdomains and this suffices to conclude $D^{s} u=0$ and $|\nabla u| \log \left(1+|\nabla u|^{2}\right) \in L_{\text {loc }}^{1}(\Omega)$.

$L \log L$-estimate. We combine the estimates from Theorem 1.10 and Theorem 1.11 (in the latter one we replace $r$ with $2 r$ and $R$ with $3 r$ ) getting

$$
\int_{B_{r}\left(x_{0}\right)}|\nabla u| \log \left(1+|\nabla u|^{2}\right) d x \leqq C\left(1+\frac{1}{r^{n+1}} \int_{B_{3 r}\left(x_{0}\right)}|u| d x\right) \int_{B_{2 r}\left(x_{0}\right)}(1+|\nabla u|) d x .
$$

Now we would like to apply Poincaré's inequality but this is not immediately possible since the mean value $u_{x_{0}, 3 r}$ of $u$ on $B_{3 r}\left(x_{0}\right)$ need not vanish. However, the function $u-u_{x_{0}, 3 r}$ is still a generalized minimizer with respect to its own boundary values on $B_{3 r}\left(x_{0}\right)$ and thus the last estimate still holds if we replace $u$ by $u-u_{x_{0}, 3 r}$. Now we are in the position to apply Poincaré's inequality, and we conclude the claimed estimate.

Uniqueness. Once the regularity part of the corollary is proved, uniqueness follows. Actually, we may repeat the simple argument from the end of the proof of Theorem 1.10.

5.4. The set of generalized minimizers. In this subsection we will work explicitly with the definition of $\mathscr{F}^{\mathscr{D}}$ in order to prove Theorem 1.16 . Moreover, we will finally establish Theorem 1.3.

For the moment we just suppose that $f: \mathbb{R}^{N n} \rightarrow[0, \infty)$ is convex with (H1) and for $u_{0} \in W^{1,1}\left(\Omega, \mathbb{R}^{N}\right)$ we write as usual

$$
\mathscr{D}=u_{0}+W_{0}^{1,1}\left(\Omega, \mathbb{R}^{N}\right)
$$

for the corresponding Dirichlet class.

Let us start with simple observations about the set of generalized minimizers.

Lemma 5.4. For any $u \in B V\left(\Omega, \mathbb{R}^{N}\right)$ the set of possible additive constants

$$
Y:=\left\{y \in \mathbb{R}^{N}: u+y \text { is a generalized minimizer of } F \text { in } \mathscr{D}\right\}
$$

is convex, closed, and bounded in $\mathbb{R}^{N}$.

20) Assuming $\lambda \geqq \Gamma$ (which is not restrictive) the assumption $f(0) \leqq \lambda$ in Theorem 1.10 and Theorem 1.11 is valid. 
Proof. All properties will be derived from the definition of $\mathscr{F}^{\mathscr{D}}$ in (1.8). First, since $f$ and $f^{\infty}$ are convex, also the functional $\mathscr{F}^{\mathscr{D}}$ is convex and the convexity of $Y$ follows. Now let us consider a sequence $y_{k} \rightarrow y$ in $\mathbb{R}^{N}$. Then lower semicontinuity of $f^{\infty}$ and Fatou's lemma give

$$
\mathscr{F}^{\mathscr{D}}[u+y] \leqq \liminf _{k \rightarrow \infty} \mathscr{F}^{\mathscr{D}}\left[u+y_{k}\right]
$$

where $y_{k}$ and $y$ occur only in the third term in (1.8). Thus $y_{k} \in Y$ implies $y \in Y$, and $Y$ is closed. Finally, if $\left|y_{k}\right| \rightarrow \infty$, then Fatou's lemma gives

$$
\liminf _{k \rightarrow \infty} \mathscr{F}^{\mathscr{D}}\left[u+y_{k}\right]=\infty
$$

which is impossible for $y_{k} \in Y$. Consequently, $Y$ is bounded.

Lemma 5.5. Suppose that generalized minimizers of $F$ in $\mathscr{D}$ are unique up to additive constants. If one minimizer $u$ attains the boundary values, i.e., $u=u_{0}$ on $\partial \Omega$, then minimizers are fully unique.

Proof. For $0 \neq y \in \mathbb{R}^{N}$ there holds

$$
\mathscr{F}^{\mathscr{D}}[u+y]=\mathscr{F}^{\mathscr{D}}[u]+\int_{\partial \Omega} f^{\infty}\left(-y \otimes v_{\Omega}\right) d \mathscr{H}^{n-1} .
$$

The last integral is positive and thus $u+y$ is not minimizing.

Now we provide a proof of Theorem 1.16 which makes substantial use of (a particular case of) Lemma 6.2 below. We remark that $(\mathrm{H} 4)$ is involved only implicitly through this lemma.

Proof of Theorem 1.16. We recall that by assumption generalized minimizers of $F$ in $\mathscr{D}$ are unique up to additive constants. Now we fix some generalized minimizer $u$ and consider the set $Y$ defined in Lemma 5.4. We will show that $Y$ is contained in a 1-dimensional subspace of $\mathbb{R}^{N}$.

Indeed, let us assume that $Y$ is not contained in a 1-dimensional subspace. Then we can find linearly independent elements $y_{1}, y_{2} \in Y$. Since $u+y_{1}$ and $u+y_{2}$ are both generalized minimizers, by Lemma 6.2 we can write $u+J_{1} y_{1}=u_{0}$ and $u+J_{2} y_{2}=u_{0}$ on $\partial \Omega$ with some functions $J_{1}, J_{2}: \partial \Omega \rightarrow \mathbb{R}$. However, this may only happen if $J_{1}$ and $J_{2}$ vanish and thus $u=u_{0}$ holds $\mathscr{H}^{n-1}$-a.e. on $\partial \Omega$. In this situation Lemma 5.5 gives $Y=\{0\}$ and thus $Y$ is always contained in a 1-dimensional subspace.

Now, taking into account the properties of $Y$ from Lemma 5.4, it follows that $Y$ is a compact interval in $\mathbb{R}^{N}$. We define $y_{0}$ as the center point of $Y$ and may then write $Y$ as $\left\{y_{0}+t \bar{y}: t \in[-1,1]\right\}$ for some $\bar{y} \in \mathbb{R}^{N}$. Setting $\bar{u}:=u+y_{0}$ we arrive at the claim.

Proof of Theorem 1.3. In view of Corollary 1.13 and Theorem 1.16 it only remains to verify the hypotheses $(\mathrm{H} 1),(\mathrm{H} 2),(\mathrm{H} 3)$, and $(\mathrm{H} 4)$ for the density $e_{\lambda}(z)=\sqrt{\lambda^{2}+|z|^{2}}$ with $\lambda>0$. However, (H1) is obvious, (H2) can be verified by an explicit computation of $\nabla^{2} e_{\lambda}$, 
(H3) (with $\lambda=0$ ) follows from the discussion of $(1.10)$ in the introduction, and (H4) is valid by Remark 1.15 .

\section{Non-uniqueness and boundary behavior}

In this section we derive Theorem 1.17 and Theorem 1.4, and we discuss additional aspects of the boundary behavior of generalized minimizers.

We start again with a simple lemma.

Lemma 6.1. Suppose that $g$ is a strictly convex norm on $\mathbb{R}^{m}$ in the sense of Definition 1.14. If for $y_{1}, y_{2} \in \mathbb{R}^{m}$ with $y_{1} \neq 0$ there occurs equality

$$
g\left(y_{1}+y_{2}\right)=g\left(y_{1}\right)+g\left(y_{2}\right)
$$

in the triangle (or convexity) inequality of $g$, then there holds

$$
y_{2}=r y_{1} \quad \text { for some } r \geqq 0 \text {. }
$$

Proof. For $y_{2}=0$ there is nothing to prove. In the case $y_{2} \neq 0$ we assume by homogeneity $g\left(y_{2}\right)=1$. With the abbreviations

$$
\lambda:=\frac{g\left(y_{1}\right)}{1+g\left(y_{1}\right)} \quad \text { and } \quad \tilde{y}_{1}:=\frac{y_{1}}{g\left(y_{1}\right)}
$$

we have

$$
g\left(\tilde{y}_{1}\right)=1 \quad \text { and } \quad g\left(\lambda \tilde{y}_{1}+(1-\lambda) y_{2}\right)=g\left(\frac{y_{1}+y_{2}}{1+g\left(y_{1}\right)}\right)=1
$$

Now Definition 1.14 gives $y_{2}=\tilde{y}_{1}$ and the claim follows.

As usual we work in the remainder of this section with a Dirichlet class

$$
\mathscr{D}=u_{0}+W_{0}^{1,1}\left(\Omega, \mathbb{R}^{N}\right),
$$

where $u_{0} \in W^{1,1}\left(\Omega, \mathbb{R}^{N}\right)$ is fixed. Moreover, we suppose from now on that $f: \mathbb{R}^{N n} \rightarrow[0, \infty)$ is convex with (H1) and (H4). The next lemma makes substantial use of $(\mathrm{H} 4)$ and is the core of the proof of both Theorem 1.16 and Theorem 1.17 .

Lemma 6.2. Consider a generalized minimizer $u$ of $F$ in $\mathscr{D}$ and a constant $0 \neq y \in \mathbb{R}^{N}$. Then $u+y$ is another generalized minimizer of $F$ in $\mathscr{D}$ if and only if there exists some function $\tilde{J}: \partial \Omega \rightarrow \mathbb{R} \backslash(0,1)$ with the following two properties:

$$
\begin{array}{r}
\int_{\{\tilde{J} \leqq 0\}} f^{\infty}\left(-y \otimes v_{\Omega}\right) d \mathscr{H}^{n-1}=\int_{\{\tilde{J} \geqq 1\}} f^{\infty}\left(y \otimes v_{\Omega}\right) d \mathscr{H}^{n-1}, \\
u+\tilde{J} y=u_{0} \quad \mathscr{H}^{n-1} \text {-a.e. on } \partial \Omega . \\
\text { Bereitgestellt von | Universitaetsbibliothek Augsburg } \\
\text { Angemeldet } \\
\text { Heruntergeladen am | 04.02.19 08:18 }
\end{array}
$$


Proof. We introduce the abbreviation

$$
g_{x}(y):=f^{\infty}\left(y \otimes v_{\Omega}(x)\right) \text { for } y \in \mathbb{R}^{N},
$$

and note that by $(\mathrm{H} 4), g_{x}$ is a strictly convex norm on $\mathbb{R}^{N}$ for $\mathscr{H}^{n-1}$-a.e. $x \in \partial \Omega$. Now we go back to the definition of $\mathscr{F}^{\mathscr{D}}$ in $(1.8)$ and observe that the terms in $\mathscr{F}^{\mathscr{D}}[u]$ and $\mathscr{F}^{\mathscr{D}}[u+y]$ which involve only the derivatives coincide. Therefore, a necessary and sufficient condition for $u+y$ to be a minimizer is that the remaining boundary integrals are also equal; in the terminology of (6.3) this means

$$
\int_{\partial \Omega} g \bullet\left(u_{0}-u-y\right) d \mathscr{H}^{n-1}=\int_{\partial \Omega} g \bullet\left(u_{0}-u\right) d \mathscr{H}^{n-1}
$$

Now let us prove the backwards implication of the lemma by checking that (6.4) holds if a function $\tilde{J}$ with the above properties exists. Indeed, using (6.2), homogeneity, and (6.1) we find

$$
\begin{aligned}
\int_{\partial \Omega} g_{\bullet}\left(u_{0}-u-y\right) d \mathscr{H}^{n-1} & =\int_{\{\tilde{J} \leqq 0\}}(1-\tilde{J}) g \bullet(-y) d \mathscr{H}^{n-1}+\int_{\{\tilde{J} \geqq 1\}}(\tilde{J}-1) g \bullet(y) d \mathscr{H}^{n-1} \\
& =\int_{\{\tilde{J} \leqq 0\}}(-\tilde{J}) g \bullet(-y) d \mathscr{H}^{n-1}+\int_{\{\tilde{J} \geqq 1\}} \tilde{J} g \bullet(y) d \mathscr{H}^{n-1} \\
& =\int_{\partial \Omega} g \bullet\left(u_{0}-u\right) d \mathscr{H}^{n-1} .
\end{aligned}
$$

To establish the forwards implication we assume that $u+y$ is a minimizer and we thus have (6.4). By Lemma 5.4 also $u+y / 2$ is a minimizer and therefore

$$
\int_{\partial \Omega} g_{\bullet}\left(u_{0}-u-y / 2\right) d \mathscr{H}^{n-1}
$$

must also coincide with the two integrals in (6.4). Now we notice on the one hand that by the triangle inequality for $g_{\bullet}$ there holds

$$
2 g_{\bullet}\left(u_{0}-u-y / 2\right)=g_{\bullet}\left(2 u_{0}-2 u-y\right) \leqq g_{\bullet}\left(u_{0}-u\right)+g_{\bullet}\left(u_{0}-u-y\right) .
$$

On the other hand by the preceding considerations integrating both sides of (6.5) gives the same value. Thus, $\mathscr{H}^{n-1}$-a.e. we must have equality in (6.5). At points of $\partial \Omega$ where $u_{0}=u$ holds we obviously have (6.2) with $\tilde{J}=0$. Therefore, we now restrict our considerations to points with $u_{0}-u \neq 0$. At those points we infer via Lemma 6.1 that $u_{0}-u-y=R\left(u_{0}-u\right)$ holds, where $R$ is a nonnegative function (notice $R \neq 1$ ). Solving the last equation for $u_{0}-u$ we have $u_{0}-u=y /(1-R)$, and thus (6.2) holds with $\tilde{J}=1 /(1-R)$ (taking values in $\mathbb{R} \backslash[0,1)$ ). Hence, we have constructed a function $\tilde{J}$ with (6.2). Now (6.1) follows essentially by the same computation which we made for the backwards direction.

Proof of Theorem 1.17. By Theorem 1.16 the set of all generalized minimizers of $F$ in $\mathscr{D}$ may be written as

$$
\begin{array}{r}
\{\bar{u}+t \bar{y}: t \in[-1,1]\}, \\
\text { Bereitgestellt von | Universitaetsbibliothek Augsburg } \\
\text { Angemeldet } \\
\text { Heruntergeladen am | 04.02.19 08:18 }
\end{array}
$$


where $\bar{y} \neq 0$ by the non-uniqueness assumption. In particular, $\bar{u}-\bar{y}$ and $\bar{u}+\bar{y}$ are minimizers. Applying Lemma 6.2 to these two minimizers (with $2 \bar{y}$ in place of $y$ ) we come up with a function $\tilde{J}: \partial \Omega \rightarrow \mathbb{R} \backslash(0,1)$ such that $(6.1)$ and

$$
\bar{u}-\bar{y}+2 \tilde{J} \bar{y}=u_{0}
$$

hold. If we now define $(\partial \Omega)_{+}:=\{\tilde{J} \geqq 1\},(\partial \Omega)_{-}:=\{\tilde{J} \leqq 0\}$, and

$$
J:= \begin{cases}2 \tilde{J}-1 & \text { if } \tilde{J} \geqq 1 \\ 1-2 \tilde{J} & \text { if } \tilde{J} \leqq 0\end{cases}
$$

then most of the claims of Theorem 1.17 are visible. In particular, (6.1) gives (1.11).

However, even though we have by now established the inequalities $\inf _{(\partial \Omega)_{-}} J \geqq 1$ and $\inf _{(\partial \Omega)_{+}} J \geqq 1$, obtaining equality requires the following additional reasoning. In fact, if we had $\inf _{(\partial \Omega)_{+}} J>1$, then we would also have $s:=\inf _{\{\tilde{J} \geqq 1\}} \tilde{J}>1$. Consequently, we could apply (the reverse direction of) Lemma 6.2 (with the minimizer $\bar{u}-\bar{y}$, the constant $2 s \bar{y}$, and the function $\tilde{J} / s)$ to conclude that $\bar{u}-\bar{y}+2 s \bar{y}$ is a minimizer. However, by the characterization of the set of all minimizers from the beginning of the proof, $\bar{u}-\bar{y}+2 s \bar{y}$ is not a minimizer and thus we must have $\inf _{(\partial \Omega)_{+}} J=1$. A similar argument gives $\inf _{(\partial \Omega)_{-}} J=1$.

Proof of Theorem 1.4. As it was already mentioned in the proof of Theorem 1.3 at the end of Section 5 the integrands $e_{\lambda}$ satisfy all the relevant hypotheses $(\mathrm{H} 1),(\mathrm{H} 2),(\mathrm{H} 3)$, (H4). Moreover, (1.12) is obviously satisfied by $e_{\lambda}$ and uniqueness up to constants (which was implicitly assumed) holds by Theorem 1.3. Thus, all the hypotheses of Theorem 1.17 and Remark 1.18 are available and Theorem 1.4 follows as a particular case of these statements.

In the remainder of this section we deal with the size of the sets where the boundary data are attained. We first record the following simple consequence of Theorem 1.17:

Corollary 6.3. Assume that we are in the situation of Theorem 1.16 with $\bar{y} \neq 0$. Then all generalized minimizers, apart from the extremal ones $\bar{u} \pm \bar{y}$, nowhere attain the boundary data, that is,

$$
\bar{u}+t \bar{y} \neq u_{0} \quad \mathscr{H}^{n-1} \text {-a.e. on } \partial \Omega \text { for all } t \in(-1,1) .
$$

In view of Corollary 6.3 it only remains to study the boundary behavior of the extremal minimizers $\bar{u} \pm \bar{y}$. To fix notation let us write down the two alternative situations,

$$
\begin{array}{rrrrr}
\text { either } & \bar{y}=0 & \text { and } & \mathscr{H}^{n-1}\left(\partial \Omega \cap\left\{\bar{u}=u_{0}\right\}\right) & =\lambda \mathscr{H}^{n-1}(\partial \Omega) \\
\text { or } & \bar{y} \neq 0 & \text { and } & \mathscr{H}^{n-1}\left(\partial \Omega \cap\left\{\bar{u} \pm \bar{y}=u_{0}\right\}\right) & =\lambda_{ \pm} \mathscr{H}^{n-1}(\partial \Omega),
\end{array}
$$

where $\lambda \in[0,1]$ is arbitrary, and $\lambda_{+}, \lambda_{-} \in[0,1)$ are such that $\lambda_{+}+\lambda_{-} \leqq 1$ holds (note that the cases $\lambda_{+}=1$ and $\lambda_{-}=1$ are ruled out by Lemma 5.5$)$. We now show by a modification 
of Santi's counterexample (see [74] and Section 3.3) that indeed all the situations in (6.6) may occur.

Proposition 6.4. Let $n=2, N=1$ and let us continue using the terminology of Theorem 1.16. Given arbitrary numbers $\lambda \in[0,1]$ and $\lambda_{+}, \lambda_{-} \in[0,1)$ with $\lambda_{+}+\lambda_{-} \leqq 1$ each of the two situations in (6.6) occurs with these given parameters for some bounded Lipschitz domain $\Omega$, some smooth integrand $f: \mathbb{R}^{2} \rightarrow[0, \infty)$ satisfying $(\mathrm{H} 1),(\mathrm{H} 2),(\mathrm{H} 3)$, and $(\mathrm{H} 4)$, and some Dirichlet class $\mathscr{D}=u_{0}+W_{0}^{1,1}(\Omega)$.

Remark 6.5. The following proof shows that the examples for $\bar{y}=0$ already work for the model integral $E_{1}$, while for $\bar{y} \neq 0$ examples for $E_{1}$ can only be constructed if $\max \left\{\lambda_{+}, \lambda_{-}\right\} \leqq 1 / 2$ holds. In view of Theorem 1.4 the latter restriction in the case $\bar{y} \neq 0$ is necessary since each of the sets $(\partial \Omega)_{+}$and $(\partial \Omega)_{-}$is half of $\partial \Omega$ and moreover we have

$$
\partial \Omega \cap\left\{\bar{u} \pm \bar{y}=u_{0}\right\} \subset(\partial \Omega)_{ \pm} .
$$

Proof of Proposition 6.4. The proof is divided into two parts, which correspond to the two alternative situations in (6.6). Both parts are based on the following basic strategy. We begin with Santi's example of non-uniqueness. More specifically, we consider the generalized minimizers $\hat{u}+y$ from Proposition 3.11 (or some variant). Then we modify-a posteriori-the boundary values, and we infer from Lemma 2.9 that $\hat{u}+y$ is still minimizing, for suitable $y$, with respect to the new boundary values. We will see below that the construction can be adjusted in such a way that for the modified problem each of the situations in (6.6) occurs.

Part 1 is concerned with the first of the two situations in (6.6). Given an arbitrary $\lambda \in[0,1]$ we work with Santi's two-dimensional domain $\Omega_{S}$ from Section 3.3 and we decompose the boundary $\partial \Omega_{S}$ into

$$
\partial_{+} \Omega_{S}:=\left\{x \in \partial \Omega_{S}: x_{1} x_{2}>0\right\} \quad \text { and } \quad \partial_{-} \Omega_{S}:=\left\{x \in \partial \Omega_{S}: x_{1} x_{2}<0\right\} .
$$

Moreover, we choose $\tilde{u}_{0} \in W^{1,1}\left(\Omega_{S}\right)$ with $\tilde{u}_{0}= \pm M$ on $\partial_{ \pm} \Omega_{S}$ for some constant $M>2$ which is fixed in the following. By Proposition 3.11 (applied to the model integral $E_{1}$ ) there exists a generalized minimizer $\hat{u}$ of $E_{1}$ in $\tilde{\mathscr{D}}:=\tilde{u}_{0}+W_{0}^{1,1}\left(\Omega_{S}\right)$ with $\sup _{\Omega_{S}}|\hat{u}| \leqq 2$. Now we choose an $\mathscr{H}^{1}$-measurable function $\hat{J}: \partial \Omega_{S} \rightarrow[0, M-2]$ such that we have

$$
\mathscr{H}^{1}\left(\partial \boldsymbol{\Omega}_{S} \cap\{\hat{\boldsymbol{J}}=0\}\right)=\lambda \mathscr{H}^{1}\left(\partial \boldsymbol{\Omega}_{S}\right),
$$

and additionally we require that the essential infimum of $\hat{J}$ is 0 on both $\partial_{+} \Omega_{S}$ and $\partial_{-} \Omega_{S}$. Then we take some $u_{0} \in W^{1,1}\left(\Omega_{S}\right)$ with

$$
u_{0}(x)=\hat{u}(x) \pm \hat{J}(x) \text { for } \mathscr{H}^{1} \text {-a.e. } x \in \partial_{ \pm} \Omega_{S} .
$$

By construction $u_{0}$ is a convex combination of $\hat{u}$ and $\tilde{u}_{0}$ on $\partial \Omega_{S}$, and Lemma 2.9 implies that $\hat{u}$ is a generalized minimizer of $E_{1}$ in $\mathscr{D}:=u_{0}+W_{0}^{1,1}\left(\Omega_{S}\right)$. Moreover, from the definition of $u_{0}$ and the choice of $\hat{J}$ we have

$$
\mathscr{H}^{1}\left(\partial \Omega_{S} \cap\left\{\hat{u}=u_{0}\right\}\right)=\lambda \mathscr{H}^{1}\left(\partial \Omega_{S}\right) .
$$


It remains to reason that $\hat{u}$ is the unique generalized minimizer in $\mathscr{D}$. Let us assume that this were not the case. Then, taking without loss of generality $\bar{y}>0$ we decompose $\partial \Omega_{S}$ into the disjoint sets $\left(\partial \Omega_{S}\right)_{+}$from Theorem 1.4, and we infer from that theorem that the generalized minimizer $\hat{u}$ is bounded away from $u_{0}$ on at least one of the sets $\left(\partial \Omega_{S}\right)_{+}$. In particular, $\hat{u}$ satisfies $\hat{u}<u_{0}$ on $\left(\partial \Omega_{S}\right)_{+}$or $\hat{u}>u_{0}$ on $\left(\partial \Omega_{S}\right)_{-}$. In view of $\hat{u} \leqq u_{0}$ on $\partial_{+} \Omega_{S}$ and $\hat{u} \geqq u_{0}$ on $\partial_{-} \Omega_{S}$ this implies that one of the inclusions $\left(\partial \Omega_{S}\right)_{+} \subset \partial_{ \pm} \Omega_{S}$ holds. Since the sets $\left(\partial \Omega_{S}\right)_{ \pm}$and $\partial_{ \pm} \Omega_{S}$ have the same measure, we in fact have equality $\left(\partial \Omega_{S}\right)_{ \pm}=\partial_{ \pm} \Omega_{S}$ up to a set of $\mathscr{H}^{1}$-measure zero. Consequently, $\hat{u}$ is bounded away from $u_{0}$ on one of the sets $\partial_{+} \Omega_{S}$ which contradicts the above choice of $\hat{J}$. In conclusion, we must have uniqueness, in other words $\bar{y}=0$.

Part 2 deals with the second situation in (6.6). Before proving the general claim we briefly mention that in the case $\max \left\{\lambda_{+}, \lambda_{-}\right\} \leqq 1 / 2$ one may work with a slight modification of the arguments from Part 1. Actually, this reasoning leads - in accordance with Remark 6.5-to an example for the model integral $E_{1}$ on $\Omega_{S}$.

Next we treat the general case, with arbitrary numbers $\lambda_{ \pm} \in[0,1)$ such that $\lambda_{+}+\lambda_{-} \leqq 1$ holds. Reversing the sign of $\bar{y}$ in (6.6) corresponds to interchanging the roles of $\lambda_{+}$and $\lambda_{-}$, and thus it suffices to treat the case $\lambda_{+} \leqq 1 / 2$ in the following. We now use the 1-parameter family of domains $\Omega_{S}^{r}$ from the beginning of Section 3.3, and we let

$$
\partial_{+} \Omega_{S}^{r}:=\left\{x \in \partial \Omega_{S}^{r}: x_{1} x_{2}>0\right\} \quad \text { and } \quad \partial_{-} \Omega_{S}^{r}:=\left\{x \in \partial \Omega_{S}^{r}: x_{1} x_{2}<0\right\}
$$

In the following we modify the shape of $\Omega_{S}^{r}$ by a linear transformation in order to adjust the ratio between the sizes of $\partial_{+} \Omega_{S}^{r}$ and $\partial_{-} \Omega_{S}^{r}$. We consider the endomorphism

$$
L_{r}:=\frac{1}{2}\left(\begin{array}{rr}
r+\frac{1}{r} & r-\frac{1}{r} \\
r-\frac{1}{r} & r+\frac{1}{r}
\end{array}\right)
$$

of $\mathbb{R}^{2}$ (with eigenvalues $r$ and $1 / r$, corresponding eigenvectors $(1,1)$ and $(1,-1)$, and determinant 1), and we are interested in the transformed domain $L_{r} \Omega_{S}^{r}$. We claim that there exists an $r \geqq 1$ such that we have

$$
\frac{\mathscr{H}^{1}\left(L_{r} \partial_{+} \Omega_{S}^{r}\right)}{\mathscr{H}^{1}\left(L_{r} \partial \Omega_{S}^{r}\right)} \geqq \lambda_{+} \quad \text { and } \quad \frac{\mathscr{H}^{1}\left(L_{r} \partial_{-} \Omega_{S}^{r}\right)}{\mathscr{H}^{1}\left(L_{r} \partial \Omega_{S}^{r}\right)} \geqq \lambda_{-} .
$$

In order to establish (6.7) we record that the four points $( \pm 1 / 4, \pm 1 / 4)$ are contained in $\partial \Omega_{S}^{r}$ for all values of $r$. Moreover, the intersections of $\partial \Omega_{S}^{r}$ with the coordinate axes can be written as $\left( \pm \xi_{r}, 0\right)$ and $\left(0, \pm \xi_{r}\right)$ with some $\xi_{r}>1 / 2$, and explicit computations give $\lim _{r \rightarrow \infty} \xi_{r}=1 / 2$. Hence $\Omega_{S}^{r}$ converges to the square $Q:=\left\{x \in \mathbb{R}^{2}:\left|x_{1}\right|+\left|x_{2}\right|<1 / 2\right\}$ as $r \rightarrow \infty$.

We now compare $L_{r} \Omega_{S}^{r}$ and the rectangle $L_{r} Q$ with edges of length $r / \sqrt{2}$ and $1 /(\sqrt{2} r)$. Without going into detailed computations let us record that the length of the 
arcs of $L_{r} \partial \Omega_{S}^{r}$ exceeds the length of the corresponding edges of $L_{r} \partial Q$ at most by $2 r\left(\xi_{r}-1 / 2\right)$, and we thus have

$$
\frac{\mathscr{H}^{1}\left(L_{r} \partial_{+} \Omega_{S}^{r}\right)}{\mathscr{H}^{1}\left(L_{r} \partial \Omega_{S}^{r}\right)} \leqq \frac{2 \frac{1}{\sqrt{2} r}+4 r\left(\xi_{r}-\frac{1}{2}\right)}{2 \frac{r}{\sqrt{2}}+2 \frac{1}{\sqrt{2} r}} \underset{r \rightarrow \infty}{\longrightarrow} 0 .
$$

Moreover, the quantity $\frac{\mathscr{H}^{1}\left(L_{r} \partial_{+} \Omega_{S}^{r}\right)}{\mathscr{H}^{1}\left(L_{r} \partial \Omega_{S}^{r}\right)}$ depends continuously on $r$ and takes the value $1 / 2$ for $r=1$. Thus, for $\lambda_{+}>0$ (remember $\lambda_{+} \leqq 1 / 2$ ) we always find an $r \geqq 1$ with

$$
\frac{\mathscr{H}^{1}\left(L_{r} \partial_{+} \Omega_{S}^{r}\right)}{\mathscr{H}^{1}\left(L_{r} \partial \Omega_{S}^{r}\right)}=\lambda_{+}
$$

and because of $\lambda_{+}+\lambda_{-} \leqq 1$ the assertions in (6.7) follow. Furthermore, for $\lambda_{+}=0$ we choose an $r \geqq 1$ with $\frac{\mathscr{H}^{1}\left(L_{r} \partial_{+} \Omega_{S}^{r}\right)}{\mathscr{H}^{1}\left(L_{r} \partial \Omega_{S}^{r}\right)} \leqq 1-\lambda_{-}$and (6.7) follows also in this case.

From now on we may thus fix $r \geqq 1$ with (6.7). It is not difficult to check that the assertions of Proposition 3.11 still hold for the integral $E_{1}$ on $\Omega_{S}^{r}$ (instead of $\Omega_{S}$ ), with some bound $M_{0}$, depending only on $r$, in place of $2 f(0)$. Then composing all the functions under consideration with $L_{r}^{-1}$ we transform the minimization problem to $L_{r} \Omega_{S}^{r}$, and we come out with the following statement. We introduce the integrand

$$
\tilde{e}_{1}(z):=\sqrt{1+\left|z L_{r}\right|^{2}} \text { for } z \in \mathbb{R}^{n}
$$

which satisfies (H1), (H2), (H3), and (H4), and for $w \in W^{1,1}\left(L_{r} \Omega_{S}^{r}\right)$ we consider

$$
\tilde{E}_{1}[w]:=\int_{L_{r} \Omega_{S}^{r}} \tilde{e}_{1}(\nabla w(x)) d x=\int_{\Omega_{S}^{r}} \sqrt{1+\left|\nabla\left(w \circ L_{r}\right)\right|^{2}} d x
$$

where the last equality exploits $\operatorname{det} L_{r}=1$. Fixing $M>M_{0}+1$ for the remainder of the proof we choose $\tilde{u}_{0} \in W^{1,1}\left(L_{r} \Omega_{S}^{r}\right)$ such that $\tilde{u}_{0}(x)= \pm M$ holds on $L_{r} \partial_{ \pm} \Omega_{S}^{r}$, and we set $\tilde{\mathscr{D}}:=\tilde{u}_{0}+W_{0}^{1,1}\left(L_{r} \Omega_{S}^{r}\right)$. Then there exists a generalized minimizer $\hat{u}$ of $\tilde{E}_{1}$ in $\tilde{\mathscr{D}}$ such that $\sup |\hat{u}| \leqq M_{0}$ holds. Moreover, $\hat{u}+y$ is minimizing in $\mathscr{D}$ for every $y \in \mathbb{R}$ with $|y| \leqq 1$. $L_{r} \Omega_{S}^{r}$

Finally, we conclude the proof similarly to Part 1. In view of (6.7) we choose an $\mathscr{H}^{1}$-measurable function $\hat{J}: L_{r} \partial \Omega_{S}^{r} \rightarrow\left[1, M-M_{0}\right]$ such that we have

$$
\mathscr{H}^{1}\left(L_{r} \partial_{ \pm} \Omega_{S}^{r} \cap\{\hat{J}=1\}\right)=\lambda_{ \pm} \mathscr{H}^{1}\left(L_{r} \partial \Omega_{S}^{r}\right),
$$

and additionally ${ }^{21)}$ we require that the essential infimum of $\hat{J}$ is 1 on both $L_{r} \partial_{+} \Omega_{S}^{r}$ and $L_{r} \partial_{-} \Omega_{S}^{r}$. Taking some $u_{0} \in W^{1,1}\left(L_{r} \Omega_{S}^{r}\right)$ with

$$
u_{0}(x)=\hat{u}(x) \pm \hat{J}(x) \quad \text { for } \mathscr{H}^{1} \text {-a.e. } x \in L_{r} \partial_{ \pm} \Omega_{S}^{r}
$$

21) The requirement for the infima is relevant only if $\lambda_{+}=0$ or $\lambda_{-}=0$ holds. 
we find that $u_{0}$ is a convex combination of $\hat{u}+y$ and $\tilde{u}_{0}$ on $\partial \Omega_{S}$, and by Lemma $2.9, \hat{u}+y$ is a generalized minimizer of $\tilde{E}_{1}$ in $\mathscr{D}:=u_{0}+W_{0}^{1,1}\left(L_{r} \Omega_{S}^{r}\right)$, for every $y \in \mathbb{R}$ with $|y| \leqq 1$. In particular, we have non-uniqueness, that is $\bar{y} \neq 0$. Additionally, by the choice of $u_{0}$ and $\hat{J}$ we have

$$
\mathscr{H}^{1}\left(L_{r} \partial \Omega_{S}^{r} \cap\left\{\hat{u} \pm 1=u_{0}\right\}\right)=\lambda_{ \pm} \mathscr{H}^{1}\left(L_{r} \partial \Omega_{S}^{r}\right)
$$

Thus it just remains to argue that $\hat{u}+1$ and $\hat{u}-1$ are extremal minimizers. However, if one of them were not extremal, then by Theorem 1.17 it would be bounded away from $u_{0}$ on $L_{r} \partial \Omega_{S}^{r}$ which contradicts the above requirement for the infima of $\hat{J}$. In conclusion, we have constructed an example such that the second situation in (6.6) occurs, and the proof is complete.

\section{A. (Semi-)Continuity and existence}

This section is concerned with the functional $\mathscr{F}^{\mathscr{D}}$ from (1.8), where

$$
\mathscr{D}=u_{0}+W_{0}^{1,1}\left(\Omega, \mathbb{R}^{N}\right)
$$

with $u_{0} \in W^{1,1}\left(\Omega, \mathbb{R}^{N}\right)$ is a fixed Dirichlet class. In the following Theorem A.1 we summarize continuity properties of $\mathscr{F}^{\mathscr{D}}$, which are essentially known from [43]. Then we give a proof of these properties, which is based on (a supplement to) Theorem 2.4 and follows the lines of [43]. Finally, we derive Theorem 1.8 as a corollary of Theorem A.1 combined with Lemma 2.3 .

Theorem A.1. Suppose that $f: \mathbb{R}^{N n} \rightarrow[0, \infty)$ is convex with $(\mathrm{H} 1)$ and that $\left(u_{k}\right)_{k \in \mathbb{N}}$ is a sequence in $B V\left(\Omega, \mathbb{R}^{N}\right)$ which converges in $L^{1}\left(\Omega, \mathbb{R}^{N}\right)$ to some $u \in B V\left(\Omega, \mathbb{R}^{N}\right)$.

Semicontinuity part. There holds

$$
\mathscr{F}^{\mathscr{D}}[u] \leqq \liminf _{k \rightarrow \infty} \mathscr{F}^{\mathscr{D}}\left[u_{k}\right]
$$

Continuity part. If the equality

$$
\left|\left(\mathscr{L}^{n}, D u\right)\right|(\Omega)+\int_{\partial \Omega}\left|u_{0}-u\right| d \mathscr{H}^{n-1}=\lim _{k \rightarrow \infty}\left[\left|\left(\mathscr{L}^{n}, D u_{k}\right)\right|(\Omega)+\int_{\partial \Omega}\left|u_{0}-u_{k}\right| d \mathscr{H}^{n-1}\right]
$$

is valid, then there holds

$$
\mathscr{F}^{\mathscr{D}}[u]=\lim _{k \rightarrow \infty} \mathscr{F}^{\mathscr{D}}\left[u_{k}\right]
$$

Moreover, if $f: \mathbb{R}^{N n} \rightarrow[0, \infty)$ is strictly convex ${ }^{22)}$ and $f^{\infty}$ is a strictly convex norm, then the reverse implication is also true.

22) The strict convexity of $f$ is meant in the usual sense, that is $f\left(\lambda z_{1}+(1-\lambda) z_{2}\right)<\lambda f\left(z_{1}\right)+(1-\lambda) f\left(z_{2}\right)$ for all $\lambda \in(0,1)$ and $z_{1} \neq z_{2}$ in $\mathbb{R}^{N n}$. 
Next we briefly sketch a proof of Theorem A.1 which is based on the following ideas from [43]. Fix a bounded Lipschitz domain $\tilde{\Omega}$ containing $\bar{\Omega}$ and a $W^{1,1}$-extension $\tilde{u}_{0}$ of $u_{0}$ from $\Omega$ to $\tilde{\Omega}$. For all $w \in B V\left(\Omega, \mathbb{R}^{N}\right)$ define $\tilde{w} \in B V\left(\tilde{\Omega}, \mathbb{R}^{N}\right)$ by

$$
\tilde{w}:= \begin{cases}w & \text { on } \Omega \\ \tilde{u}_{0} & \text { on } \tilde{\Omega} \backslash \Omega\end{cases}
$$

Then Corollary 3.89 of [6] provides the formula

$$
D \tilde{w}=D w+\left(u_{0}-w\right) \otimes v_{\Omega} 1_{\partial \Omega} \cdot \mathscr{H}^{n-1}+\nabla u_{0} \mathbb{1}_{\tilde{\Omega} \backslash \Omega} \cdot \mathscr{L}^{n}
$$

where $D w$ is viewed as a measure on $\tilde{\Omega}$ with support in $\Omega$. Hence, $\mathscr{F}^{\mathscr{D}}[w]$ can be writtenin contrast to $(2.7)$ we here include the boundary integral in $\mathscr{F}^{\mathscr{D}}$-as

$$
\text { (A.2) } \quad \mathscr{F}^{\mathscr{D}}[w]=\int_{\tilde{\Omega}} \bar{f}\left(\frac{d\left(\mathscr{L}^{n}, D \tilde{w}\right)}{d\left|\left(\mathscr{L}^{n}, D \tilde{w}\right)\right|}\right) d\left|\left(\mathscr{L}^{n}, D \tilde{w}\right)\right|-\int_{\tilde{\Omega} \backslash \Omega} f\left(\nabla \tilde{u}_{0}\right) d x
$$

where $\bar{f}$ is the function from (2.6), and where the last integral is independent of $w$. Having represented $\mathscr{F}^{\mathscr{D}}$ in this way we give a

Proof of the semicontinuity part of Theorem A.1. We assume $\sup _{k \in \mathbb{N}} \mathscr{F}^{\mathscr{D}}\left[u_{k}\right]<\infty$. Then by the coercivity condition in $(\mathrm{H} 1)$ it follows that $\tilde{u}_{k}$ converges to $\tilde{u}$ weakly-* in $B V\left(\Omega, \mathbb{R}^{N}\right)$. In view of (A.2) we may apply Theorem 2.4 and Remark 2.5 to $\mathscr{F} \mathscr{D}$, and we infer that $\mathscr{F}^{\mathscr{D}}[u] \leqq \liminf _{k \rightarrow \infty} \mathscr{F}^{\mathscr{D}}\left[u_{k}\right]$ holds.

Before proving the continuity part of Theorem A.1 we deal with a lemma concerning a strict convexity property of $\bar{f}$.

Lemma A.2. Suppose that $f: \mathbb{R}^{N n} \rightarrow[0, \infty)$ is strictly convex with $(\mathrm{H} 1)$ and that $f^{\infty}$ is a strictly convex norm. Then the function $\bar{f}$ from (2.6) is a strictly convex 1-homogeneous function, where the strictness is to be understood in the following sense: The implication ${ }^{23)}$

$$
\bar{f}\left(\chi_{1}+\chi_{2}\right)=\bar{f}\left(\chi_{1}\right)+\bar{f}\left(\chi_{2}\right) \Rightarrow \chi_{1}=0 \text { or } \chi_{2}=r \chi_{1} \text { for some } r \geqq 0
$$

holds for all $\chi_{1}, \chi_{2} \in[0, \infty) \times \mathbb{R}^{N n}$.

Proof. According to Remark 2.5, $\bar{f}$ is convex and 1-homogeneous, so it remains to establish (A.3). To this end we assume that the hypothesis of (A.3) holds for $\chi_{1}=\left(t_{1}, z_{1}\right)$ and $\chi_{2}=\left(t_{2}, z_{2}\right)$ in $[0, \infty) \times \mathbb{R}^{N n}$, and we distinguish the following cases. If $t_{1}=t_{2}=0$ holds, the claim follows from (2.6) and Lemma 6.1. If $t_{1}>0$ and $t_{2}>0$ hold, then the strict convexity of $f$ gives $z_{1} / t_{1}=z_{2} / t_{2}$ as claimed since otherwise the computation

23) The convexity property in (A.3) is closely related to the strict convexity of norms; see Definition 1.14 and Lemma 6.1. However, $\bar{f}$ need not be positive outside $\{0\}$ and thus_even if we extend it onto $\mathbb{R}^{1+N n}$-it need not be a norm. 


$$
\begin{aligned}
\bar{f}\left(\chi_{1}+\chi_{2}\right) & =\left(t_{1}+t_{2}\right) f\left(\frac{t_{1}}{t_{1}+t_{2}} \cdot \frac{z_{1}}{t_{1}}+\frac{t_{2}}{t_{1}+t_{2}} \cdot \frac{z_{2}}{t_{2}}\right) \\
& <t_{1} f\left(\frac{z_{1}}{t_{1}}\right)+t_{2} f\left(\frac{z_{2}}{t_{2}}\right)=\bar{f}\left(\chi_{1}\right)+\bar{f}\left(\chi_{2}\right)
\end{aligned}
$$

would result in a contradiction. In the case $t_{1}>0=t_{2}$ we have by 1 -homogeneity and convexity

$$
\begin{aligned}
2 \bar{f}\left(\chi_{1}+\chi_{2}\right) & =\bar{f}\left(2 t_{1}, 2 z_{1}+2 z_{2}\right) \leqq \bar{f}\left(t_{1}, z_{1}\right)+\bar{f}\left(t_{1}, z_{1}+2 z_{2}\right) \\
& \leqq \bar{f}\left(t_{1}, z_{1}\right)+\bar{f}\left(t_{1}, z_{1}\right)+\bar{f}\left(0,2 z_{2}\right)=2\left[\bar{f}\left(\chi_{1}\right)+\bar{f}\left(\chi_{2}\right)\right]
\end{aligned}
$$

However, by assumption the terms on the very left and the very right of the latter estimate coincide and in particular we must have $\bar{f}\left(2 t_{1}, 2 z_{1}+2 z_{2}\right)=\bar{f}\left(t_{1}, z_{1}\right)+\bar{f}\left(t_{1}, z_{1}+2 z_{2}\right)$. From the previous case we then get $z_{2}=0$. Hence, we arrive at $\chi_{2}=0$, and thus the claim is established also for $t_{1}>0=t_{2}$. Finally, the case $t_{2}>0=t_{1}$ follows by exchange of the variables.

Moreover, we record a supplement to Theorem 2.4 which was also obtained in $[73]^{24)}$; compare [43], Theorem 1.6, [10], Theorem 2.1, and [54], Theorem 3. The following version involving cones has not been stated up to now but is a direct outcome of the respective proofs; see for instance Theorem 2.38 and Theorem 2.39 in [6].

Theorem A.3. Consider a sequence $\left(\mu_{k}\right)_{k \in \mathbb{N}}$ of finite $\mathbb{R}^{m}$-valued Radon measures on $\Omega$ which converges weakly-* to a finite $\mathbb{R}^{m}$-valued Radon measure $\mu$ on $\Omega$. Moreover, assume that $\mu_{k}$ and $\mu$ take values in some closed convex cone $K$ in $\mathbb{R}^{m}$. If

$$
\int_{\Omega} \bar{f}\left(\frac{d \mu}{d|\mu|}\right) d|\mu|=\lim _{k \rightarrow \infty} \int_{\Omega} \bar{f}\left(\frac{d \mu_{k}}{d\left|\mu_{k}\right|}\right) d\left|\mu_{k}\right|
$$

holds for one strictly convex 1-homogeneous function $\bar{f}: K \rightarrow[0, \infty)$ in the sense of (A.3) (with $K$ in place of $[0, \infty) \times \mathbb{R}^{N n}$ ), then (A.4) holds for all continuous and 1-homogeneous functions $\bar{f}: K \rightarrow[0, \infty)$.

Proof of the continuity part of Theorem A.1. We first recall (A.1) and (A.2), and we record that if one of the two equalities in the continuity part of Theorem A.1 holds, then $\tilde{u}_{k}$ converges to $\tilde{u}$ weakly-* in $B V\left(\Omega, \mathbb{R}^{N}\right)$. Thus, we deduce from (A.2) and Theorem A.3 that $\mathscr{F}^{\mathscr{D}}$ is continuous along $\left(u_{k}\right)_{k \in \mathbb{N}}$ for every integrand $f$, once it is shown to be continuous for one integrand $f$ such that the corresponding function $\bar{f}$ is strictly convex in the sense of (A.3). In turn, (A.3) is available by Lemma A.2 if $f$ is strictly convex with (H1) and $f^{\infty}$ is a strictly convex norm.

Since the last properties are valid for the integrand $e_{1}$ from Section 1.1, the above reasoning gives in particular

$$
\mathscr{E}_{1}^{\mathscr{D}}[u]=\lim _{k \rightarrow \infty} \mathscr{E}_{1}^{\mathscr{D}}\left[u_{k}\right] \Rightarrow \mathscr{F}^{\mathscr{D}}[u]=\lim _{k \rightarrow \infty} \mathscr{F}^{\mathscr{D}}\left[u_{k}\right]
$$

24) The reader should note that a mistake in the translation of the respective statement from [73] was pointed out in [10]. 
for every $f: \mathbb{R}^{N n} \rightarrow[0, \infty)$ which is convex with $(\mathrm{H} 1)$. Moreover, if $f$ is even strictly convex and $f^{\infty}$ is a strictly convex norm, then the reverse implication in (A.5) also holds. Rewriting the first two terms in the definition (1.3) of $\mathscr{E}_{1}^{\mathscr{D}}$ as in Remark 2.5 we have proven the claims.

Proof of Theorem 1.8. We first assume that an arbitrary $u \in B V\left(\Omega, \mathbb{R}^{N}\right)$ is given and we work with the approximating sequence $\left(w_{k}\right)_{k \in \mathbb{N}}$ from Lemma 2.3 . Then by the continuity part of Theorem A.1 we get

$$
\mathscr{F}^{\mathscr{D}}[u]=\lim _{k \rightarrow \infty} \mathscr{F}^{\mathscr{D}}\left[w_{k}\right]=\lim _{k \rightarrow \infty} F\left[w_{k}\right]
$$

We have thus shown that ' $\geqq$ ' holds in (1.9). However, the reverse inequality is trivially valid and (1.9) is proved.

It remains to establish the claimed characterization. gives

If $u$ is a generalized minimizer of $F$ in $\mathscr{D}$, then the above reasoning via Lemma 2.3

$$
\lim _{k \rightarrow \infty} F\left[w_{k}\right]=\mathscr{F}^{\mathscr{D}}[u]=\inf _{\mathscr{D}} F,
$$

and hence $w_{k}$ is a minimizing sequence for $F$ in $\mathscr{D}$ which converges to $u$ in $L^{1}\left(\Omega, \mathbb{R}^{N}\right)$.

Conversely, if we consider a minimizing sequence for $F$ in $\mathscr{D}$, converging in $L^{1}\left(\Omega, \mathbb{R}^{N}\right)$ to $u \in B V\left(\Omega, \mathbb{R}^{N}\right)$, then by the semicontinuity part of Theorem A.1 we have $\mathscr{F}^{\mathscr{D}}[u] \leqq \inf _{\mathscr{D}} F$. In view of $(1.9), u$ is a generalized minimizer of $F$ in $\mathscr{D}$.

\section{B. Additional remarks on the Dirichlet problem}

In this section we further investigate the minimization problem for the integral

$$
F[w]:=\int_{\Omega} f(\nabla w) d x
$$

from (1.1) in a Dirichlet class

$$
\mathscr{D}=u_{0}+W_{0}^{1,1}\left(\Omega, \mathbb{R}^{N}\right)
$$

with $u_{0} \in W^{1,1}\left(\Omega, \mathbb{R}^{N}\right)$. We provide some additional statements which are essentially consequences of Corollary 1.13 and Theorem 2.4. Though there are no innovative arguments in this section, it seems that the results do not occur explicitly in the literature, not even in the scalar case or for area minimizing graphs.

As a common feature the following statements are based on the assumption that some function $u \in B V\left(\Omega, \mathbb{R}^{N}\right)$, mostly a generalized minimizer, satisfies

$$
u=u_{0} \quad \text { in the sense of trace on } \partial \Omega \text {. }
$$


Unfortunately, apart from those for area minimizing graphs (compare the end of Section 1.2 and of the present section) there are no convenient criteria for having (B.1).

\section{Full uniqueness.}

Proposition B.1. Suppose that $f: \mathbb{R}^{N n} \rightarrow[0, \infty)$ is strictly convex with $(\mathrm{H} 1)$ and that $u$ is a generalized minimizer of $F$ in $\mathscr{D}$. If $u \in \mathscr{D}$ holds, then $u$ is the unique generalized minimizer of $F$ in $\mathscr{D}$.

Proof. We assume that $v \in B V\left(\Omega, \mathbb{R}^{N}\right)$ is another generalized minimizer of $F$ in $\mathscr{D}$. Then the uniqueness argument from the proof of Theorem 1.10 in Section 5.3 gives $\nabla u=\nabla v$ on $\Omega$. Since we assume $u \in \mathscr{D}$, we moreover have $D^{s} u=0$ and $u=u_{0}$ on $\partial \Omega$. Thus, from the equality $\mathscr{F}^{\mathscr{D}}[u]=\mathscr{F}^{\mathscr{D}}[v]$ we get

$$
\int_{\Omega} f^{\infty}\left(\frac{d D^{s} v}{d\left|D^{s} v\right|}\right) d\left|D^{s} v\right|+\int_{\partial \Omega} f^{\infty}\left(\left(u_{0}-v\right) \otimes v_{\Omega}\right) d \mathscr{H}^{n-1}=0
$$

Since $f^{\infty}(z)>0$ holds for $z \neq 0$, this implies $D^{s} v=0$ and $v=u_{0}$ on $\partial \Omega$. Consequently, we have $D u=D v$ and $u=v$ on $\partial \Omega$ and by the constancy theorem we deduce $u=v$ on $\Omega$.

Strong/strict convergence of minimizing sequences. The next result states that a sequence which converges in energy already converges in a good sense provided that the limit function satisfies (B.1). In fact, we supply a tripartite statement: The first part deals with strong convergence of sequences in $W^{1,1}\left(\Omega, \mathbb{R}^{N}\right)$, the second part concerns strict convergence of sequences in $B V\left(\Omega, \mathbb{R}^{N}\right)$, and the third part regards convergence of traces.

Theorem B.2. Suppose that $f: \mathbb{R}^{N n} \rightarrow[0, \infty)$ is convex with (H1). Moreover, consider a sequence $\left(u_{k}\right)_{k \in \mathbb{N}}$ in $B V\left(\Omega, \mathbb{R}^{N}\right)$ which converges in $L^{1}\left(\Omega, \mathbb{R}^{N}\right)$ to $u \in B V\left(\Omega, \mathbb{R}^{N}\right)$, and assume that there holds

$$
\mathscr{F}^{\mathscr{D}}\left[u_{k}\right] \underset{k \rightarrow \infty}{\longrightarrow} \mathscr{F}^{\mathscr{D}}[u]
$$

Finally, suppose that

$$
u=u_{0} \quad \text { on } \partial \Omega
$$

(i) If $f$ is strictly convex and the sequence $\left(u_{k}\right)_{k \in \mathbb{N}}$ and $u$ are in $W^{1,1}\left(\Omega, \mathbb{R}^{N}\right)$, then $u_{k}$ converges strongly to $u$ in $W^{1,1}\left(\Omega, \mathbb{R}^{N}\right)$.

(ii) If $f$ is strictly convex and $f^{\infty}$ is a strictly convex norm, then $u_{k}$ converges strictly to $u$ in $B V\left(\Omega, \mathbb{R}^{N}\right)$ (in the sense of Definition 2.2).

(iii) The trace of $u_{k}$ converges to the trace of $u$ in $L^{1}\left(\partial \Omega, \mathbb{R}^{N} ; \mathscr{H}^{n-1}\right)$.

Before proving Theorem B.2 we apply it to minimizing sequences:

Corollary B.3 (strong/strict convergence of minimizing sequences). Suppose that $f: \mathbb{R}^{N n} \rightarrow[0, \infty)$ is strictly convex with $(\mathrm{H} 1)$ and that $u$ is a generalized minimizer of $F$ 
in $\mathscr{D}$ with

$$
u=u_{0} \quad \text { on } \partial \Omega
$$

(i) If $u$ is in $W^{1,1}\left(\Omega, \mathbb{R}^{N}\right)$, then every minimizing sequence for $F$ in $\mathscr{D}$ converges strongly to $u$ in $W^{1,1}\left(\Omega, \mathbb{R}^{N}\right)$.

(ii) If $f^{\infty}$ is a strictly convex norm, then there exists a minimizing sequence for $F$ in $\mathscr{D}$ which converges strictly to $u$ in $B V\left(\Omega, \mathbb{R}^{N}\right)$.

Remark B.4. Under additional hypotheses the $W^{1,1}$-assumption in Corollary B.3 (i) is guaranteed by Theorem 1.10; compare Corollary B.6 below.

Proof of Corollary B.3. To establish (i) we show that every minimizing sequence for $F$ in $\mathscr{D}$ has a subsequence converging to $u$ in $W^{1,1}\left(\Omega, \mathbb{R}^{N}\right)$. To prove this claim we first exploit the coercivity of $f$ and conclude that every minimizing sequence has a subsequence converging in $L^{1}\left(\Omega, \mathbb{R}^{N}\right)$ to some limit $v \in B V\left(\Omega, \mathbb{R}^{N}\right)$. By Theorem 1.8, $v$ is a generalized minimizer of $F$ in $\mathscr{D}$, and if $u \in \mathscr{D}$ holds, then Proposition B.1 gives $u=v$. At this point Theorem B.2 (i) guarantees strong convergence $u_{k} \underset{k \rightarrow \infty}{\longrightarrow} u$ in $W^{1,1}\left(\Omega, \mathbb{R}^{N}\right)$.

The claim in (ii) follows from Theorem 1.8 and Theorem B.2 (ii).

The proof of Theorem B.2 begins with one more lemma on convex functions.

Lemma B.5. Suppose that $f: \mathbb{R}^{m} \rightarrow \mathbb{R}$ is strictly convex and consider $z \in \mathbb{R}^{m}$ and a sequence $\left(z_{k}\right)_{k \in \mathbb{N}}$ in $\mathbb{R}^{m}$. If $f(z)+f\left(z_{k}\right)-2 f\left(\frac{z_{k}+z}{2}\right)$ converges to 0 , then $z_{k}$ converges
to $z$.

Proof. For ease of notation let us assume $z=0$. As a straightforward consequence of the convexity inequality for $f$,

$$
[0, \infty) \rightarrow \mathbb{R}, \quad r \mapsto f(r \xi)-2 f\left(r \frac{\xi}{2}\right) \text { is non-decreasing }
$$

for every $\xi \in \mathbb{R}^{m}$. Now let us assume that $z_{k}$ does not converge to 0 . Possibly passing to a subsequence we then have $\left|z_{k}\right| \geqq \varepsilon$ for some $\varepsilon>0$, and $z_{k} /\left|z_{k}\right| \rightarrow \omega$ for some $\omega \in \mathbb{R}^{m}$ with $|\omega|=1$. Exploiting (B.2) we conclude

$$
\begin{aligned}
f(0)+f\left(z_{k}\right)-2 f\left(\frac{z_{k}}{2}\right) & \geqq f(0)+f\left(\varepsilon \frac{z_{k}}{\left|z_{k}\right|}\right)-2 f\left(\varepsilon \frac{z_{k}}{2\left|z_{k}\right|}\right) \\
& \underset{k \rightarrow \infty}{\longrightarrow} f(0)+f(\varepsilon \omega)-2 f\left(\varepsilon \frac{\omega}{2}\right) .
\end{aligned}
$$

By the strict convexity of $f$ the limit on the right-hand side of the previous formula is positive, and thus $f(0)+f\left(z_{k}\right)-2 f\left(\frac{z_{k}}{2}\right)$ does not converge to 0 . 
Proof of Theorem B.2 (i). Due to the assumption $u=u_{0}$ on $\partial \Omega$ the boundary integrals in the expression $\mathscr{F}^{\mathscr{D}}[u]+\mathscr{F}^{\mathscr{D}}\left[u_{k}\right]-2 \mathscr{F}^{\mathscr{D}}\left[\frac{u_{k}+u}{2}\right]$ cancel out. Moreover, by the
semicontinuity part of Theorem A.1 we have

$$
\lim _{k \rightarrow \infty} \mathscr{F}^{\mathscr{D}}\left[u_{k}\right]=\mathscr{F}^{\mathscr{D}}[u] \leqq \liminf _{k \rightarrow \infty} \mathscr{F}^{\mathscr{D}}\left[\frac{u_{k}+u}{2}\right] .
$$

All in all—exploiting $D^{s} u=0=D^{s} u_{k}$ —we get

$$
\begin{aligned}
\limsup _{k \rightarrow \infty} \int_{\Omega}\left[f(\nabla u)+f\left(\nabla u_{k}\right)-2 f\left(\frac{\nabla u_{k}+\nabla u}{2}\right)\right] d x \\
=\mathscr{F}^{\mathscr{D}}[u]+\lim _{k \rightarrow \infty} \mathscr{F}^{\mathscr{D}}\left[u_{k}\right]-2 \liminf _{k \rightarrow \infty} \mathscr{F}^{\mathscr{D}}\left[\frac{u_{k}+u}{2}\right]=0 .
\end{aligned}
$$

However, by the convexity of $f$ the integrands $f(\nabla u)+f\left(\nabla u_{k}\right)-2 f\left(\frac{\nabla u_{k}+\nabla u}{2}\right)$ are nonnegative and hence converge to 0 in $L^{1}(\Omega)$. Passing once more to a subsequence we infer convergence pointwise a.e. on $\Omega$, and by Lemma B.5 we deduce $\nabla u_{k} \rightarrow \nabla u$ a.e. on $\Omega$. Fatou's lemma (note that (H1) gives $f\left(\nabla u_{k}\right)+f(\nabla u)-\gamma\left|\nabla u_{k}-\nabla u\right| \geqq 0$ ) yields

$$
\begin{aligned}
2 \mathscr{F}^{\mathscr{D}}[u]=\int_{\Omega} 2 f(\nabla u) d x & \leqq \liminf _{k \rightarrow \infty} \int_{\Omega}\left[f\left(\nabla u_{k}\right)+f(\nabla u)-\gamma\left|\nabla u_{k}-\nabla u\right|\right] d x \\
& \leqq \lim _{k \rightarrow \infty} \mathscr{F}^{\mathscr{D}}\left[u_{k}\right]+\mathscr{F}^{\mathscr{D}}[u]-\gamma \limsup _{k \rightarrow \infty} \int_{\Omega}\left|\nabla u_{k}-\nabla u\right| d x \\
& =2 \mathscr{F}^{\mathscr{D}}[u]-\gamma \limsup _{k \rightarrow \infty} \int_{\Omega}\left|\nabla u_{k}-\nabla u\right| d x .
\end{aligned}
$$

In conclusion, we obtain strong convergence $u_{k} \underset{k \rightarrow \infty}{\longrightarrow} u$ in $W^{1,1}\left(\Omega, \mathbb{R}^{N}\right)$ as claimed.

Proof of Theorem B.2 (ii). From the coercivity condition in (H1) we deduce that $u_{k}$ converges to $u$ not only in $L^{1}\left(\Omega, \mathbb{R}^{N}\right)$ but also weakly- $*$ in $B V\left(\Omega, \mathbb{R}^{N}\right)$. Therefore it suffices to prove

$$
\lim _{k \rightarrow \infty}\left|D u_{k}\right|(\Omega)=|D u|(\Omega)
$$

To this end we apply the continuity part of Theorem A.1 twice: By the backwards implication we find

$$
\left|\left(\mathscr{L}^{n}, D u\right)\right|(\Omega)+\int_{\partial \Omega}\left|u_{0}-u\right| d \mathscr{H}^{n-1}=\lim _{k \rightarrow \infty}\left[\left|\left(\mathscr{L}^{n}, D u_{k}\right)\right|(\Omega)+\int_{\partial \Omega}\left|u_{0}-u_{k}\right| d \mathscr{H}^{n-1}\right],
$$

and then using the forwards implication for the integrand $e_{0}$ from Section 1.1 we arrive at

$$
\mathscr{E}_{0}^{\mathscr{D}}[u]=\lim _{k \rightarrow \infty} \mathscr{E}_{0}^{\mathscr{D}}\left[u_{k}\right]
$$


Taking into account the definition $(1.3)$ and $u=u_{0}$ on $\partial \Omega$ the last equality just means

$$
|D u|(\Omega)=\lim _{k \rightarrow \infty}\left[\left|D u_{k}\right|(\Omega)+\int_{\partial \Omega}\left|u_{0}-u_{k}\right| d \mathscr{H}^{n-1}\right] .
$$

By the semicontinuity of the variation (which is a special case of Theorem 2.4) we also know

$$
|D u|(\Omega) \leqq \liminf _{k \rightarrow \infty}\left|D u_{k}\right|(\Omega)
$$

Combining the last two formulas we end up with (B.3).

Under the strict convexity assumptions of part (ii) of Theorem B.2 the preceding arguments also yield the convergence of traces. The following arguments establish this convergence in the more general setting of part (iii).

Proof of Theorem B.2 (iii). As in the proof of part (ii), $u_{k}$ converges to $u$ weakly-* in $B V\left(\Omega, \mathbb{R}^{N}\right)$. Thus, we may apply Theorem 2.4 as explained in Remark 2.5 to the measures $D u_{k}$ coming out with

$$
\begin{aligned}
& \int_{\Omega} f(\nabla u) d x+\int_{\Omega} f^{\infty}\left(\frac{d D^{s} u}{d\left|D^{s} u\right|}\right) d\left|D^{s} u\right| \\
& \quad \leqq \liminf _{k \rightarrow \infty}\left[\int_{\Omega} f\left(\nabla u_{k}\right) d x+\int_{\Omega} f^{\infty}\left(\frac{d D^{s} u_{k}}{d\left|D^{s} u_{k}\right|}\right) d\left|D^{s} u_{k}\right|\right] .
\end{aligned}
$$

Moreover, exploiting the hypothesis $u=u_{0}$ on $\partial \Omega$ we find

$$
\begin{aligned}
\int_{\Omega} f(\nabla u) d x+\int_{\Omega} f^{\infty}\left(\frac{d D^{s} u}{d\left|D^{s} u\right|}\right) d\left|D^{s} u\right| \\
=\mathscr{F}^{\mathscr{D}}[u] \\
=\lim _{k \rightarrow \infty} \mathscr{F}^{\mathscr{D}}\left[u_{k}\right] \\
=\liminf _{k \rightarrow \infty}\left[\int_{\Omega} f\left(\nabla u_{k}\right) d x+\int_{\Omega} f^{\infty}\left(\frac{d D^{s} u_{k}}{d\left|D^{s} u_{k}\right|}\right) d\left|D^{s} u_{k}\right|\right] \\
\quad+\limsup _{k \rightarrow \infty} \int_{\partial \Omega} f^{\infty}\left(\left(u_{0}-u_{k}\right) \otimes v_{\Omega}\right) d \mathscr{H}^{n-1} .
\end{aligned}
$$

Combining the last formulas we arrive at

$$
\lim _{k \rightarrow \infty} \int_{\partial \Omega} f^{\infty}\left(\left(u_{0}-u_{k}\right) \otimes v_{\Omega}\right) d \mathscr{H}^{n-1}=0
$$

Since $u=u_{0}$ holds on $\partial \Omega$, the left-hand inequality in (H1) gives the claimed convergence. 
Continuous dependence on the boundary data. We now record another result on strong convergence which follows from the regularity result in Corollary 1.13 combined with Theorem B.2. An abstract reformulation of the result will be given below.

Corollary B.6. Suppose that $f: \mathbb{R}^{N n} \rightarrow[0, \infty)$ is $C^{2}$ with $(\mathrm{H} 1),(\mathrm{H} 2)$, and $(\mathrm{H} 3)$, and that $\left(u_{0 ; k}\right)_{k \in \mathbb{N}}$ is a sequence in $W^{1,1}\left(\Omega, \mathbb{R}^{N}\right)$ such that the trace of $u_{0 ; k}$ converges to the trace of $u_{0}$ in $L^{1}\left(\partial \Omega, \mathbb{R}^{N} ; \mathscr{H}^{n-1}\right)$. Moreover, assume that $u_{k}$ and $u$ are generalized minimizers of $F$ in $\mathscr{D}_{k}:=u_{0 ; k}+W_{0}^{1,1}\left(\Omega, \mathbb{R}^{N}\right)$ and $\mathscr{D}=u_{0}+W_{0}^{1,1}\left(\Omega, \mathbb{R}^{N}\right)$, respectively, and that

$$
u=u_{0} \quad \text { on } \partial \Omega
$$

Then $u_{k}$ converges strongly to $u$ in $W^{1,1}\left(\Omega, \mathbb{R}^{N}\right)$.

Proof. We first note that by Corollary 1.13 the sequence $\left(u_{k}\right)_{k \in \mathbb{N}}$ and $u$ are in $W^{1,1}\left(\Omega, \mathbb{R}^{N}\right)$. In particular, since we are also assuming $u=u_{0}$ on $\partial \Omega$, we have $u \in \mathscr{D}$. Moreover, by Lemma $2.7, f^{\infty}$ is Lipschitz continuous and thus there holds

$$
\left|\mathscr{F}^{\mathscr{D}_{k}}[w]-\mathscr{F}^{\mathscr{D}}[w]\right| \leqq L \int_{\partial \Omega}\left|u_{0 ; k}-u_{0}\right| d \mathscr{H}^{n-1} \quad \text { for all } w \in B V\left(\Omega, \mathbb{R}^{N}\right),
$$

where the positive constant $L$ depends only on $N n$ and $\Gamma$. Using this together with the minimality of $u_{k}$ we get

$$
\begin{aligned}
\mathscr{F}^{\mathscr{D}}\left[u_{k}\right] & \leqq \mathscr{F}^{\mathscr{D}_{k}}\left[u_{k}\right]+L \int_{\partial \Omega}\left|u_{0 ; k}-u_{0}\right| d \mathscr{H}^{n-1} \\
& \leqq \mathscr{F}^{\mathscr{D} k}[u]+L \int_{\partial \Omega}\left|u_{0 ; k}-u_{0}\right| d \mathscr{H}^{n-1} \\
& \leqq \mathscr{F}^{\mathscr{D}}[u]+2 L \int_{\partial \Omega}\left|u_{0 ; k}-u_{0}\right| d \mathscr{H}^{n-1}
\end{aligned}
$$

Now the assumed convergence of the traces and the minimality of $u$ give

$$
\mathscr{F}^{\mathscr{D}}\left[u_{k}\right] \underset{k \rightarrow \infty}{\longrightarrow} \mathscr{F}^{\mathscr{D}}[u]=\inf _{B V\left(\Omega, \mathbb{R}^{N}\right)} \mathscr{F}^{\mathscr{D}} .
$$

Exploiting the coercivity in $(\mathrm{H} 1)$ and passing to a subsequence we deduce that $u_{k}$ converges in $L^{1}\left(\Omega, \mathbb{R}^{N}\right)$. By the semicontinuity part of Theorem A.1 the limit function is a generalized minimizer and by the uniqueness result in Proposition B.1 it coincides with $u$. Now we are in the position to apply Theorem B.2 (i) which yields the claim.

Imposing the assumptions of Corollary B.6 on the integrand $f$ we are interested in maps which assign to a given Dirichlet class $\mathscr{D}=u_{0}+W_{0}^{1,1}\left(\Omega, \mathbb{R}^{N}\right)$-more precisely to the trace of $u_{0}$ which determines $\mathscr{D}$-a generalized minimizer of $F$ in $\mathscr{D}$. By Corollary 1.13 these generalized minimizers are in $W^{1,1}\left(\Omega, \mathbb{R}^{N}\right)$ and unique up to constants, but in general they are not fully unique. However, there are several ways of designating a specific generalized minimizer corresponding to each class, for instance if additionally (H4) holds, we may take the minimizer $\bar{u}$ from Theorem 1.16. All possible ways of choosing generalized minimizers are represented by resolvent operators

$$
R: L^{1}\left(\partial \Omega, \mathbb{R}^{N} ; \mathscr{H}^{n-1}\right) \rightarrow W^{1,1}\left(\Omega, \mathbb{R}^{N}\right)
$$


defined on the trace space which have the following property for all $u_{0} \in W^{1,1}\left(\Omega, \mathbb{R}^{N}\right)$ :

$R$ maps the trace of $u_{0}$ onto a generalized minimizer of $F$ in $u_{0}+W_{0}^{1,1}\left(\Omega, \mathbb{R}^{N}\right)$.

Moreover, let us introduce the class $A_{f ; \Omega}$ of attained boundary values in $L^{1}\left(\partial \Omega, \mathbb{R}^{N} ; \mathscr{H}^{n-1}\right)$, that is, the set of all traces of functions $u_{0} \in W^{1,1}\left(\Omega, \mathbb{R}^{N}\right)$ such that some generalized minimizer of $F$ in $u_{0}+W_{0}^{1,1}\left(\Omega, \mathbb{R}^{N}\right)$ coincides with $u_{0}$ on $\partial \Omega$. In view of Lemma 2.9 we may equivalently say that $A_{f ; \Omega}$ is the set of all traces of generalized minimizers of $F$, in all Dirichlet classes in $W^{1,1}\left(\Omega, \mathbb{R}^{N}\right)$.

With this terminology Corollary B.6 can be rephrased-keeping in mind Proposition B.1 - by saying that the traces in $A_{f ; \Omega}$ are continuity points of the resolvent operators:

Corollary B.7. Suppose that $f$ satisfies the hypothesis of Corollary B.6. Moreover, consider a resolvent operator $R$ in the above sense and endow both $L^{1}\left(\partial \Omega, \mathbb{R}^{N} ; \mathscr{H}^{n-1}\right)$ and $W^{1,1}\left(\Omega, \mathbb{R}^{N}\right)$ with the strong topology. Then $R$ is continuous at all points of $A_{f ; \Omega}$.

We briefly comment on the composition $\nabla \circ R$ with the gradient operator, that is, we map onto the gradient of a generalized minimizer instead of the minimizer itself. The map

$$
\nabla \circ R: L^{1}\left(\partial \Omega, \mathbb{R}^{N} ; \mathscr{H}^{n-1}\right) \rightarrow L^{1}\left(\Omega, \mathbb{R}^{N n}\right)
$$

is also continuous at all points of $A_{f ; \Omega}$. But moreover $\nabla \circ R$ is uniquely determined by $f$ and $\Omega$, and thus it might be the most reasonable object to study in this context.

The Dirichlet problem with $\boldsymbol{L}^{\mathbf{1}}$ data. In the remainder of this section we assume that the hypotheses of Corollary B.6 are valid for some given integrand $f$. We recall that as the crucial assumption of this section we assumed that a generalized minimizer $u$ of $F$ in $\mathscr{D}=u_{0}+W_{0}^{1,1}\left(\Omega, \mathbb{R}^{N}\right)$ satisfies (B.1), that is, $u=u_{0}$ on $\partial \Omega$. If (B.1) holds, then by Corollary 1.13 we have $u \in \mathscr{D}$. Consequently, $u$ is a minimizer in the usual sense (i.e., $u$ realizes the minimum not only on the left-hand side but even on the right-hand side of (1.9)) and moreover the preceding results apply to $u$. In view of all these properties we think that it is natural to ask for which Dirichlet classes $\mathscr{D}$ (B.1) holds. Since $\mathscr{D}$ is determined by the trace of $u_{0}$, this question can be reformulated in the terminology of the preceding paragraph as follows:

What can be said about the class $A_{f ; \Omega}$ of attained boundary values?

Let us summarize what is known about this question. For particular choices of $f$ and $\Omega$ and for certain symmetric boundary data it is possible to perform a reduction to a 1-dimensional problem as in Sections 3.2 and 3.3, and to decide in this way whether the boundary data are in $A_{f ; \Omega}$ or not. A contribution which goes beyond such particular symmetric situations is Miranda's boundary continuity result for area minimizing graphs in the scalar case $N=1$; see [66], [67]. This result implies that if $\partial \Omega$ has nonnegative mean curvature, then $A_{e_{1} ; \Omega}$ contains all traces which are $\mathscr{H}^{n-1}$-a.e. continuous. An interesting example, which highlights the sharpness of Miranda's result, was provided by Baldo and Modica [11]. For the two-dimensional unit ball $B_{1}^{2}$ they constructed a bounded and everywhere discontinuous boundary datum which is not in $A_{e_{1} ; B_{1}^{2}}$. In particular, this implies that $A_{e_{1} ; B_{1}^{2}}$ is strictly smaller than $L^{1}\left(\partial B_{1}^{2}, \mathbb{R}^{N} ; \mathscr{H}^{n-1}\right)$. In our opinion it would be interesting to 
investigate in more detail the attainment of $L^{1}$ boundary data and the class $A_{e_{1} ; B_{1}^{2}}$ - or even the classes $A_{f ; \Omega}$ with more general $f$ and $\Omega$.

\section{Non-autonomous integrals}

Here, we briefly discuss variational integrals depending explicitly on the independent variable, that is, we consider functionals of the form

$$
F[w]:=\int_{\Omega} f(\cdot, \nabla w) d x \quad \text { for } w: \Omega \rightarrow \mathbb{R}^{N}
$$

with an integrand $f: \bar{\Omega} \times \mathbb{R}^{N n} \rightarrow[0, \infty)$. Basically, the results in this paper extend to this more general setting without requiring significantly new ideas if the assumptions $(\mathrm{H} 1),(\mathrm{H} 2)$, (H3), and all notions of convexity are imposed on the functions $f(x, \cdot)$, uniformly in $x$. In particular, with an analogous definition of generalized minimizers the (semi-)continuity results of Appendix A, the characterization of generalized minimizers in Theorem 1.8, and the existence result of Corollary 1.9 carry over almost ${ }^{25)}$ verbatim; compare [73], [43], [7], $[6]$.

In order to state a generalization of Theorem 1.10 we follow [23], Section 3 (see also [16], Chapter 4.2.2.2), and we impose the following additional assumptions on the derivatives of $f$ :

$$
\begin{gathered}
\left|\frac{\partial}{\partial x_{i}} \nabla_{z} f(x, z)\right| \leqq \Gamma, \\
\left|\frac{\partial^{2}}{\partial x_{i}^{2}} \nabla_{z} f(x, z)\right| \leqq \Gamma, \\
\left|\frac{\partial}{\partial x_{i}} \nabla_{z}^{2} f(x, z)\left(\tilde{z}_{1}, \tilde{z}_{2}\right)\right| \leqq \Gamma\left[\left|\nabla_{z}^{2} f(x, z)\left(\tilde{z}_{1}, \tilde{z}_{2}\right)\right|+(1+|z|)^{-2}\left|\tilde{z}_{1}\right|\left|\tilde{z}_{2}\right|\right]
\end{gathered}
$$

for all $z, \tilde{z}_{1}, \tilde{z}_{2} \in \mathbb{R}^{N n}, x \in \Omega$ and $i \in\{1, \ldots, n\}$.

Theorem C.1. Assume that $f: \bar{\Omega} \times \mathbb{R}^{N n} \rightarrow[0, \infty)$ is continuous and that (H1) and (H2) hold for all functions $f(x, \cdot)$, uniformly in $x \in \Omega$. Moreover, suppose that (C.1), (C.2), and (C.3) are valid, where all the occurring derivatives exist and are continuous on $\Omega \times \mathbb{R}^{N n}$. Then bounded generalized minimizers of $F$ in $\mathscr{D}$ are unique up to additive constants. Furthermore, each such generalized minimizer $u \in B V\left(\Omega, \mathbb{R}^{N}\right) \cap L^{\infty}\left(\Omega, \mathbb{R}^{N}\right)$ satisfies

$$
u \in W^{1,1}\left(\Omega, \mathbb{R}^{N}\right) \quad \text { and } \quad|\nabla u| \log \left(1+|\nabla u|^{2}\right) \in L_{\mathrm{loc}}^{1}(\Omega) .
$$

Theorem C. 1 can be proved following the strategy of Section 5 and handling the additional terms as in [23], Section 3. The crucial point is to provide variants of Lemma 5.1

25) In the non-autonomous case one assumes additionally that the functions $f(x, z)$ and $f^{\infty}(x, z)$ are (lower semi-)continuous in $(x, z)$. 
and Lemma 5.2. Here, (C.1) is needed in order to establish Lemma 5.1, while (C.2) and (C.3) are used to get uniform estimates for some additional terms in the proof of Lemma 5.2. We omit further details.

As for Theorem 1.10, the boundedness assumption in Theorem C.1 can be derived either from the maximum principles in Appendix D or from a straightforward adaptation of Section 4 to integrals with $x$-dependency.

Remark C.2. It is not possible to weaken the assumption (H2) in Theorem C.1. Actually, if $(\mathrm{H} 2)$ is replaced by $(\mathrm{H} 2 \mu)$ with some $\mu>3$, then by virtue of a counterexample in [23], Section 4 (based on previous ideas in [43]) generalized minimizers need not be in $W^{1,1}$. However, it is not clear whether it is possible to construct a corresponding counterexample in the autonomous setting.

\section{Maximum principles}

Next we briefly discuss two maximum principles (and the corresponding minimum principles) for minimizers in $W^{1,1}\left(\Omega, \mathbb{R}^{N}\right)$ and generalized minimizers in $B V\left(\Omega, \mathbb{R}^{N}\right)$, respectively. These principles apply to component functions of minimizers in the vectorial case and work under quite weak assumptions on the integrands - even though we do not intend to state them in maximal generality. The first, quite simple principle is proved by a standard argument (compare [29]). The second principle for generalized minimizers is somewhat more involved but still follows the same idea. Our main interest is in a corollary of the second principle (Corollary D.3) which provides the $L^{\infty}$-bound needed in Theorem 1.10 and Theorem C.1. integrals

Now we start by stating the principles for minimizers in $W^{1,1}\left(\Omega, \mathbb{R}^{N}\right)$. We consider

$$
F[w]:=\int_{\Omega} f(\cdot, \nabla w) d x \quad \text { for } w \in W^{1,1}\left(\Omega, \mathbb{R}^{N}\right)
$$

with a Borel function $f: \Omega \times \mathbb{R}^{N n} \rightarrow[0, \infty)$. We fix $\alpha \in\{1,2, \ldots, N\}$ and we write $z^{\alpha} \in \mathbb{R}^{n}$ for the $\alpha$ th row of $z$. We assume that whenever we fix some $x \in \Omega$ and all the entries of $z$ except those in $z^{\alpha}$ then

(D.2) the function $\mathbb{R}^{n} \rightarrow \mathbb{R}, z^{\alpha} \mapsto f(x, z)$, has a unique minimum at 0 .

In particular, (D.2) is satisfied if $f$ is strictly convex and even in $z^{\alpha}$. Comparing this with the conditions of Section 1.2 we record that either of the conditions (1.10) and (1.12) implies even dependence on all variables.

Theorem D.1. Assume that $f: \Omega \times \mathbb{R}^{N n} \rightarrow[0, \infty)$ is a Borel function which satisfies (D.2) for some fixed $\alpha \in\{1,2, \ldots, N\}$. Moreover, suppose that $u \in W^{1,1}\left(\Omega, \mathbb{R}^{N}\right)$ is a minimizer of the integral $F$ from (D.1), that is, $F[u] \leqq F[w]$ for all $w \in u+W_{0}^{1,1}\left(\Omega, \mathbb{R}^{N}\right)$. Then one has

$$
\sup _{\Omega} u^{\alpha}=\sup _{\partial \Omega} u^{\alpha} \quad \text { and } \quad \inf _{\Omega} u^{\alpha}=\inf _{\partial \Omega} u^{\alpha}
$$


Proof. We only prove the maximum principle. By a standard property of traces we have $\sup _{\partial \Omega} u^{\alpha} \leqq \sup _{\Omega} u^{\alpha}$. Hence, if $M:=\sup _{\partial \Omega} u^{\alpha}$ is infinite, there is nothing to prove. Otherwise we compare $u$ with $w \in W^{1,1}\left(\Omega, \mathbb{R}^{N}\right)$ defined by

$$
w^{\beta}:= \begin{cases}u^{\beta} & \text { for } \beta \neq \alpha \\ \min \left\{u^{\alpha}, M\right\} & \text { for } \beta=\alpha\end{cases}
$$

Then we have $\nabla w^{\alpha}=\mathbb{1}_{\left\{u^{\alpha} \leqq M\right\}} \cdot \nabla u^{\alpha}$, and thus it holds $\mathscr{L}^{n}$-a.e. on $\Omega$ either $\nabla w^{\alpha}=\nabla u^{\alpha}$ or $\nabla w^{\alpha}=0 \neq \nabla u^{\alpha}$. Additionally, we evidently have $\nabla w^{\beta}=\nabla u^{\beta}$ for $\beta \neq \alpha$. Using the assumption (D.2) we infer

(D.3) $\quad f(\cdot, \nabla w) \leqq f(\cdot, \nabla u) \quad \mathscr{L}^{n}$-a.e. on $\Omega$ with equality only where $\nabla u=\nabla w$.

Moreover, since $u^{\alpha} \leqq M$ holds on $\partial \Omega$, we have $w=u$ on $\partial \Omega, w$ is admissible as a comparison function for the minimality property, and we have

$$
\int_{\Omega} f(\cdot, \nabla u) d x \leqq \int_{\Omega} f(\cdot, \nabla w) d x
$$

In view of the last inequality we must have equality in (D.3), and thus $\nabla u=\nabla w$ holds $\mathscr{L}^{n}$-a.e. on $\Omega$. Taking into account $u=w$ on $\partial \Omega$ it follows that $u^{\alpha}=w^{\alpha} \leqq M$ holds $\mathscr{L}^{n}$-a.e. on $\Omega$.

Next we state the principles for generalized minimizers in $B V\left(\Omega, \mathbb{R}^{N}\right)$. To this end we consider integrals

$$
\begin{aligned}
\mathscr{F}[w]:= & \int_{\Omega} f(\cdot, \nabla w) d x+\int_{\Omega} g\left(\cdot, \frac{d D^{s} w}{d\left|D^{s} w\right|}\right) d\left|D^{s} w\right| \\
& +\int_{\partial \Omega} g\left(\cdot,\left(u_{0}-w\right) \otimes v_{\Omega}\right) d \mathscr{H}^{n-1}
\end{aligned}
$$

with $u_{0} \in W^{1,1}\left(\Omega, \mathbb{R}^{N}\right), f$ as above, and a Borel function $g: \bar{\Omega} \times \mathbb{R}^{N n} \rightarrow[0, \infty)$, which is 1 -homogeneous in its second argument. Of course we are interested in the case where $g$ is the recession function of $f$. However, for the purposes of the next statement this relation is irrelevant and we just require that whenever we fix $x \in \bar{\Omega}$ and all the entries of $z$ except those in $z^{\alpha}$ then

(D.5) the function $\mathbb{R}^{n} \rightarrow \mathbb{R}, z^{\alpha} \mapsto g(x, z)$, is radially strictly increasing.

Reformulating (D.5) as a formula we require that for all $x \in \bar{\Omega}$ and $z, \xi \in \mathbb{R}^{N n}$ we have the implication

$$
\left.\begin{array}{c}
\xi^{\beta}=z^{\beta} \text { for } \beta \neq \alpha, \\
z^{\alpha} \neq 0, \xi^{\alpha}=t z^{\alpha} \text { with some } t \in[0,1)
\end{array}\right\} \Rightarrow g(x, \xi)<g(x, z) .
$$

In particular, (D.5) is satisfied if $g$ is a strictly convex norm (in the sense of Definition 1.14) in $z$ and even in $z^{\alpha}$. 
Theorem D.2. We fix $u_{0} \in W^{1,1}\left(\Omega, \mathbb{R}^{N}\right), \quad \alpha \in\{1,2, \ldots, N\}$, a Borel function $f: \Omega \times \mathbb{R}^{N n} \rightarrow[0, \infty)$ with (D.2), and a Borel function $g: \bar{\Omega} \times \mathbb{R}^{N n} \rightarrow[0, \infty)$ which is 1 -homogeneous in the second argument and satisfies (D.5). Moreover, we suppose that $u \in B V\left(\Omega, \mathbb{R}^{N}\right)$ minimizes $\mathscr{F}$ from $(\mathrm{D} .4)$, that is, $\mathscr{F}[u] \leqq \mathscr{F}[w]$ for all $w \in B V\left(\Omega, \mathbb{R}^{N}\right)$. Then we have

$$
\sup _{\Omega} u^{\alpha}=\sup _{\partial \Omega} u^{\alpha} \leqq \sup _{\partial \Omega} u_{0}^{\alpha} \quad \text { and } \quad \inf _{\Omega} u^{\alpha}=\inf _{\partial \Omega} u^{\alpha} \geqq \inf _{\partial \Omega} u_{0}^{\alpha}
$$

Proof. It suffices to establish for $M \in \mathbb{R}$ the implications

(D.6) $\quad u^{\alpha} \leqq M \quad \mathscr{H}^{n-1}$-a.e. on $\partial \Omega \Rightarrow u^{\alpha} \leqq M \quad \mathscr{L}^{n}$-a.e. on $\Omega$,

(D.7) $\quad u_{0}^{\alpha} \leqq M \quad \mathscr{H}^{n-1}$-a.e. on $\partial \Omega \Rightarrow u^{\alpha} \leqq M \quad \mathscr{L}^{n}$-a.e. on $\Omega$.

We start by assuming $u^{\alpha} \leqq M$ on $\partial \Omega$, and-proceeding similarly to the proof of Theorem D.1 - we use the comparison function $w \in B V\left(\Omega, \mathbb{R}^{N}\right)$ defined by

$$
w^{\beta}:= \begin{cases}u^{\beta} & \text { for } \beta \neq \alpha \\ \min \left\{u^{\alpha}, M\right\} & \text { for } \beta=\alpha\end{cases}
$$

We first note that $w$ can be written as the composition of $u$ with a Lipschitz function and that $D w$ can be computed by an adequate version of the chain rule. Since we only change one component function, it suffices to apply the chain rule [6], Theorem 3.99, for realvalued $^{26)}$ functions which yields the formulas

$$
\nabla w^{\alpha}=\mathbb{1}_{\left\{u^{\alpha} \leqq M\right\}} \nabla u^{\alpha}, \quad D^{c} w^{\alpha}=\mathbb{1}_{\left\{u^{\alpha} \leqq M\right\}} \cdot D^{c} u^{\alpha},
$$

and

$$
D^{j} w^{\alpha}=\frac{\min \left\{\left(u^{\alpha}\right)^{+}, M\right\}-\min \left\{\left(u^{\alpha}\right)^{-}, M\right\}}{\left(u^{\alpha}\right)^{+}-\left(u^{\alpha}\right)^{-}} \cdot D^{j} u^{\alpha},
$$

where $D^{s} w^{\alpha}=D^{c} w^{\alpha}+D^{j} w^{\alpha}$ is the decomposition into the Cantor part and the jump part and $\left(u^{\alpha}\right)^{ \pm}$are the traces of $u^{\alpha}$ on the set of approximate jump points of $u^{\alpha}$; see [6], Chapter 3, for further details. Additionally, we evidently have $D w^{\beta}=D u^{\beta}$ for $\beta \neq \alpha$.

Now we notice that (D.3) still holds by the same reasoning as in the proof of Theorem D.1. Moreover, from the formulas for $D^{c} w^{\alpha}$ and $D^{j} w^{\alpha}$ we infer that $D^{s} w$ is absolutely continuous with respect to $\left|D^{s} u\right|$ and

$$
D^{s} w^{\alpha}=\chi \cdot D^{s} u^{\alpha}
$$

for some Borel function $\chi: \Omega \rightarrow[0,1]$. By the 1-homogeneity of $g$ and assumption (D.5) we deduce

$$
\int_{\Omega} g\left(\cdot, \frac{d D^{s} w}{d\left|D^{s} w\right|}\right) d\left|D^{s} w\right|=\int_{\Omega} g\left(\cdot, \frac{d D^{s} w}{d\left|D^{s} u\right|}\right) d\left|D^{s} u\right| \leqq \int_{\Omega} g\left(\cdot, \frac{d D^{s} u}{d\left|D^{s} u\right|}\right) d\left|D^{s} u\right|
$$

with equality only if $D^{s} u=D^{s} w$.

26) In this context the chain rule for real-valued functions is much simpler than its vectorial counterpart [6], Theorem 3.101. 
Additionally, from the minimality of $u$ we have $\mathscr{F}[u] \leqq \mathscr{F}[w]$. Since $u^{\alpha} \leqq M$ and thus $w=u$ hold on $\partial \Omega$, the boundary integrals in $\mathscr{F}[u]$ and $\mathscr{F}[w]$ are the same, and in view of (D.3) and (D.8) it follows that also the other integrals coincide, that is, we have equality in (D.3) and (D.8) and consequently $D u=D w$. Taking into account $u=w$ on $\partial \Omega$ we arrive at $u^{\alpha}=w^{\alpha} \leqq M$ on $\Omega$ and (D.6) is proved.

To prove (D.7) we assume $u_{0}^{\alpha} \leqq M$ on $\partial \Omega$, we still use the comparison function $w$, and we observe that the arguments leading to (D.3) and (D.8) remain unchanged. However, this time we supply an additional argument to establish $w=u$ on $\partial \Omega$. Exploiting $u_{0}^{\alpha} \leqq M$ on $\partial \Omega$ and the definition of $w$ we get

$$
u_{0}^{\alpha}-w^{\alpha}=\tilde{\chi}\left(u_{0}^{\alpha}-u^{\alpha}\right) \quad \text { on } \partial \Omega
$$

for some $\tilde{\chi}: \partial \Omega \rightarrow[0,1]$. Applying (D.5) we then find

$$
\begin{gathered}
g\left(\cdot,\left(u_{0}-w\right) \otimes v_{\Omega}\right) \leqq g\left(\cdot,\left(u_{0}-u\right) \otimes v_{\Omega}\right) \quad \mathscr{H}^{n-1} \text {-a.e. on } \partial \Omega \\
\text { with equality only where } u=w .
\end{gathered}
$$

Now invoking the minimality property $\mathscr{F}[u] \leqq \mathscr{F}[w]$ as before we derive equality in (D.3), (D.8), and (D.9), that is, $D u=D w$ and $u=w$ on $\partial \Omega$. We infer $u^{\alpha}=w^{\alpha} \leqq M$ on $\Omega$ and (D.7) is proved.

One may formulate several variants of Theorem D.1 and Theorem D.2. Here, we provide only one more principle which is relevant in connection with Theorem 1.10 and Theorem C.1.

Corollary D.3. Suppose that the hypotheses of Theorem D.2 are valid for all $\alpha \in\{1,2, \ldots, N\}$ instead of just one. Then for $M \geqq 0$ we have

$$
\left|u_{0}\right| \leqq M \quad \mathscr{H}^{n-1} \text {-a.e. on } \partial \Omega \quad \Rightarrow \quad|u| \leqq M \sqrt{N} \quad \mathscr{L}^{n} \text {-a.e. on } \Omega \text {. }
$$

Proof. From Theorem D.2 we get $\left|u^{\alpha}\right| \leqq M$ on $\Omega$ for all component functions $u^{\alpha}$. Summing up we arrive at $|u| \leqq M \sqrt{N}$ on $\Omega$.

Acknowledgement. This project was partially supported by the Scuola Normale Superiore di Pisa via the program "Progetti 2010 Giovani Ricercatori”.

\section{References}

[1] E. Acerbi and G. Dal Maso, New lower semicontinuity results for polyconvex integrals, Calc. Var. Part. Diff. Equ. 2 (1994), 329-371.

[2] E. Acerbi and N. Fusco, A regularity theorem for minimizers of quasiconvex integrals, Arch. Ration. Mech. Anal. 99 (1987), 261-281.

[3] E. Acerbi and N. Fusco, Regularity for minimizers of non-quadratic functionals: the case $1<p<2$, J. Math. Anal. Appl. 140 (1989), 115-135.

[4] R. A. Adams and J. J. F. Fournier, Sobolev spaces, 2nd ed., Academic Press, Amsterdam 2003.

[5] L. Ambrosio and G. Dal Maso, On the relaxation in $B V\left(\Omega ; \mathbb{R}^{m}\right)$ of quasi-convex integrals, J. Funct. Anal. 109 (1992), 76-97.

[6] L. Ambrosio, N. Fusco and D. Pallara, Functions of bounded variation and free discontinuity problems, Oxford University Press, Oxford 2000. 
[7] G. Anzellotti, The Euler equation for functionals with linear growth, Trans. Am. Math. Soc. 290 (1985), 483-501.

[8] G. Anzellotti, BV solutions of quasilinear PDEs in divergence form, Comm. Part. Diff. Equ. 12 (1987), $77-122$.

[9] G. Anzellotti and M. Giaquinta, Funzioni BV e tracce, Rend. Sem. Mat. Univ. Padova 60 (1978), 1-21.

[10] G. Anzellotti and M. Giaquinta, Convex functionals and partial regularity, Arch. Ration. Mech. Anal. 102 (1988), 243-272.

[11] S. Baldo and L. Modica, Nonuniqueness of minimal graphs, Indiana Univ. Math. J. 40 (1991), no. 3, 975-983.

[12] L. Beck and T. Schmidt, Doubly degenerate variational problems in $B V$, in preparation.

[13] S. Bernstein, Sur les équations du calcul des variations, Ann. Sci. École Norm. Sup. (3) 29 (1912), 431-485.

[14] M. Bildhauer, A uniqueness theorem for the dual problem associated to a variational problem with linear growth, J. Math. Sci. (N. Y.) 115 (2003), 2747-2752; translation from Zap. Nauchn. Semin. POMI 271 (2000), 83-91.

[15] M. Bildhauer, A priori gradient estimates for bounded generalized solutions of a class of variational problems with linear growth, J. Convex Anal. 9 (2002), 117-137.

[16] M. Bildhauer, Convex variational problems, Linear, nearly linear and anisotropic growth conditions, Lect. Notes Math. 1818, Springer, Berlin 2003.

[17] M. Bildhauer, Two dimensional variational problems with linear growth, Manuscr. Math. 110 (2003), 325-342.

[18] M. Bildhauer and M. Fuchs, Regularity for dual solutions and for weak cluster points of minimizing sequences of variational problems with linear growth, J. Math. Sci. (N. Y.) 109 (2002), 1835-1850; translation from Zap. Nauchn. Semin. POMI 259 (1999), 46-66.

[19] M. Bildhauer and M. Fuchs, Partial regularity for variational integrals with $(s, \mu, q)$-growth, Calc. Var. Part. Diff. Equ. 13 (2001), 537-560.

[20] M. Bildhauer and M. Fuchs, On a class of variational integrals with linear growth satisfying the condition of $\mu$-ellipticity, Rend. Mat. Appl. (VII) 22 (2002), 249-274.

[21] M. Bildhauer and M. Fuchs, Partial regularity for a class of anisotropic variational integrals with convex hull property, Asympt. Anal. 32 (2002), 293-315.

[22] M. Bildhauer and M. Fuchs, Relaxation of convex variational problems with linear growth defined on classes of vector-valued functions, St. Petersbg. Math J. 14 (2003), 19-33; translation from Algebra Anal. 14 (2002), 26-47.

[23] M. Bildhauer and M. Fuchs, Convex variational problems with linear growth, in: Geometric analysis and nonlinear partial differential equations, Springer, Berlin (2003), 327-344.

[24] E. Bombieri, E. De Giorgi and E. Giusti, Minimal cones and the Bernstein problem, Invent. Math. 7 (1969), 243-268.

[25] E. Bombieri, E. De Giorgi and M. Miranda, Una maggiorazione a priori relativa alle ipersuperfici minimali non parametriche, Arch. Ration. Mech. Anal. 32 (1969), 255-267.

[26] M. Carozza, J. Kristensen and A. Passarelli di Napoli, Higher differentiability of minimizers of convex variational integrals, Ann. Inst. Henri Poincaré, Anal. Non Lin. 28 (2011), no. 3, 395-411.

[27] E. De Giorgi, Frontiere orientate di misura minima, Seminario di Matematica, Sc. Norm. Super. Pisa, 19601961.

[28] E. De Giorgi, Un esempio di estremali discontinue per un problema variazionale di tipo ellittico, Boll. Unione Mat. Ital. (IV) 1 (1968), 135-137.

[29] A. D'Ottavio, F. Leonetti and C. Musciano, Maximum principle for vector-valued mappings minimizing variational integrals, Atti Semin. Mat. Fis. Univ. Modena 46 (1998), Suppl., 677-683.

[30] I. Ekeland, Sur les problèmes variationnels, C. R. Acad. Sci., Paris (A) 275 (1972), 1057-1059.

[31] I. Ekeland, On the variational principle, J. Math. Anal. Appl. 47 (1974), 324-353.

[32] I. Ekeland, Nonconvex minimization problems, Bull. Am. Math. Soc. (New Ser.) 1 (1979), 443-474.

[33] L. Esposito, F. Leonetti and G. Mingione, Regularity for minimizers of functionals with $p-q$ growth, Nonlin. Diff. Equ. Appl. 6 (1999), 133-148.

[34] L. Esposito, F. Leonetti and G. Mingione, Regularity results for minimizers of irregular integrals with $(p, q)$ growth, Forum Math. 14 (2002), 245-272.

[35] L. Esposito, F. Leonetti and G. Mingione, Sharp regularity for functionals with $(p, q)$ growth, J. Diff. Equ. 204 (2004), 5-55.

[36] L. Esposito and G. Mingione, Partial regularity for minimizers of convex integrals with $L \log L$-growth, Nonlin. Diff. Equ. Appl. 7 (2000), 107-125. 
[37] H. Federer, Geometric measure theory, Springer, Berlin 1969.

[38] M. Fuchs and G. Seregin, A regularity theory for variational integrals with $L \ln L$-growth, Calc. Var. Part. Diff. Equ. 6 (1998), 171-187.

[39] N. Fusco and J. E. Hutchinson, $C^{1, \alpha}$ partial regularity of functions minimising quasiconvex integrals, Manuscr. Math. 54 (1985), 121-143.

[40] E. Gagliardo, Caratterizzazioni delle tracce sulla frontiera relative ad alcune classi di funzioni in $n$ variabili, Rend. Sem. Mat. Univ. Padova 27 (1957), 284-305.

[41] M. Giaquinta and G. Modica, Partial regularity of minimizers of quasiconvex integrals, Ann. Inst. Henri Poincaré, Anal. Non Linéaire 3 (1986), 185-208.

[42] M. Giaquinta and G. Modica, Remarks on the regularity of the minimizers of certain degenerate functionals, Manuscr. Math. 57 (1986), 55-99.

[43] M. Giaquinta, G. Modica and J. Souček, Functionals with linear growth in the calculus of variations, Commentat. Math. Univ. Carol. 20 (1979), 143-156, 157-172.

[44] M. Giaquinta, G. Modica and J. Souček, Cartesian currents in the calculus of variations II, Variational integrals, Springer, Berlin 1998.

[45] E. Giusti, Superfici cartesiane di area minima, Rend. Sem. Mat. Fis. Milano 40 (1970), 135-153.

[46] E. Giusti, Minimal surfaces and functions of bounded variation, Birkhäuser, Basel 1984.

[47] E. Giusti, Direct methods in the calculus of variation, World Scientific Publishing, Singapore 2003.

[48] E. Giusti and M. Miranda, Un esempio di soluzioni discontinue per un problema di minimo relativo ad un integrale regolare del calcolo delle variazioni, Boll. Unione Mat. Ital. (IV) 1 (1968), 219-226.

[49] C. Goffman and J. Serrin, Sublinear functions of measures and variational integrals, Duke Math. J. 31 (1964), 159-178.

[50] G. Gregori, Generalized solutions for a class of non-uniformly elliptic equations in divergence form, Comm. Part. Diff. Equ. 22 (1997), 581-617.

[51] C. Hamburger, Regularity of differential forms minimizing degenerate elliptic functionals, J. reine angew. Math. 431 (1992), 7-64.

[52] W. Hao, S. Leonardi and J. Nečas, An example of irregular solution to a nonlinear Euler-Lagrange elliptic system with real analytic coefficients, Ann. Sc. Norm. Super. Pisa, Cl. Sci. (IV) 23 (1996), 57-67.

[53] H. Jenkins and J. Serrin, The Dirichlet problem for the minimal surface equation in higher dimensions, J. reine angew. Math. 229 (1968), 170-187.

[54] J. Kristensen and F. Rindler, Relaxation of signed integral functionals in $B V$, Calc. Var. Part. Diff. Equ. 37 (2010), 29-62.

[55] H. B. Lawson and R. Osserman, Non-existence, non-uniqueness and irregularity of solutions to the minimal surface system, Acta Math. 139 (1977), 1-17.

[56] H. Lebesgue, Intégrale, longueur, aire, Thèse, 1902.

[57] P. Marcellini, On the definition and the lower semicontinuity of certain quasiconvex integrals, Ann. Inst. Henri Poincaré, Anal. Non Linéaire 3 (1986), 391-409.

[58] P. Marcellini, Regularity of minimizers of integrals of the calculus of variations with non-standard growth conditions, Arch. Ration. Mech. Anal. 105 (1989), 267-284.

[59] P. Marcellini, Regularity and existence of solutions of elliptic equations with $p, q$-growth conditions, J. Diff. Equ. 90 (1991), 1-30.

[60] P. Marcellini and G. Papi, Nonlinear elliptic systems with general growth, J. Diff. Equ. 221 (2006), 412-443.

[61] P. Marcellini and C. Sbordone, On the existence of minima of multiple integrals of the calculus of variations, J. Math. Pures Appl. (IX) 62 (1983), 1-9.

[62] M. Meier, Boundedness and integrability properties of weak solutions of quasilinear elliptic systems, J. reine angew. Math. 333 (1982), 191-220.

[63] G. Mingione and F. Siepe, Full $C^{1, \alpha}$-regularity for minimizers of integral functionals with $L \log L$-growth, Z. Anal. Anwend. 18 (1999), 1083-1100.

[64] M. Miranda, Superfici cartesiane generalizzate ed insiemi di perimetro localmente finito sui prodotti cartesiani, Ann. Sc. Norm. Super. Pisa, Sci. Fis. Mat. (III) 18 (1964), 515-542.

[65] M. Miranda, Comportamento delle successioni convergenti di frontiere minimali, Rend. Sem. Mat. Univ. Padova 38 (1967), 238-257.

[66] M. Miranda, Un principio di massimo forte per le frontiere minimali e una sua applicazione alla risoluzione del problema al contorno per l'equazione delle superfici di area minima, Rend. Sem. Mat. Univ. Padova 45 (1971), 355-366.

[67] M. Miranda, Dirichlet problem with $L^{1}$ data for the non-homogeneous minimal surface equation, Indiana Univ. Math. J. 24 (1974), 227-241. 
[68] J. Moser, A new proof of De Giorgi's theorem concerning the regularity problem for elliptic differential equations, Commun. Pure Appl. Math. 13 (1960), 457-468.

[69] J. Moser, On Harnack's theorem for elliptic differential equations, Commun. Pure Appl. Math. 14 (1961), $577-591$.

[70] J. Necas, Example of an irregular solution to a nonlinear elliptic system with analytic coefficients and conditions for regularity, Theor. Nonlin. Oper., Constr. Aspects, Proc. Int. Summer School (Berlin 1975), Akademie-Verlag, Berlin (1977), 197-206.

[71] H. R. Parks and W. P. Ziemer, Jacobi fields and regularity of functions of least gradients, Ann. Sc. Norm. Super. Pisa, Cl. Sci. (IV) 11 (1984), 505-527.

[72] A. Passarelli di Napoli and F. Siepe, A regularity result for a class of anisotropic systems, Rend. Ist. Mat. Univ. Trieste 28 (1996), 13-31.

[73] Y. G. Reshetnyak, Weak convergence of completely additive vector functions on a set, Sib. Math. J. 9 (1968), 1039-1045.

[74] E. Santi, Sul problema al contorno per l'equazione delle superfici di area minima su domini limitati qualunque, Ann. Univ. Ferrara (N), VII, 17 (1972), 13-26.

[75] C. Scheven and T. Schmidt, Asymptotically regular problems I: Higher integrability, J. Diff. Equ. 248 (2010), 745-791.

[76] T. Schmidt, Regularity of minimizers of $W^{1, p}$-quasiconvex variational integrals with $(p, q)$-growth, Calc. Var. Part. Diff. Equ. 32 (2008), 1-24.

[77] T. Schmidt, Regularity of relaxed minimizers of quasiconvex variational integrals with $(p, q)$-growth, Arch. Ration. Mech. Anal. 193 (2009), 311-337.

[78] J. Serrin, A new definition of the integral for non-parametric problems in the calculus of variations, Acta Math. 102 (1959), 23-32.

[79] J. Serrin, On the definition and properties of certain variational integrals, Trans. Am. Math. Soc. 101 (1961), $139-167$.

[80] J. Serrin, The problem of Dirichlet for quasilinear elliptic differential equations with many independent variables, Philos. Trans. R. Soc. London (A) 264 (1969), 413-496.

[81] L. Simon, Boundary regularity for solutions of the non-parametric least area problem, Ann. Math. (2) 103 (1976), 429-455.

[82] P. Sternberg, G. Williams and W. P. Ziemer, Existence, uniqueness, and regularity for functions of least gradient, J. reine angew. Math. 430 (1992), 35-60.

[83] V. Šverák and X. Yan, A singular minimizer of a smooth strongly convex functional in three dimensions, Calc. Var. Part. Diff. Equ. 10 (2000), 213-221.

[84] V. Šverák and $X$. Yan, Non-Lipschitz minimizers of smooth uniformly convex functionals, Proc. Natl. Acad. Sci. USA 99 (2002), 15269-15276.

[85] K. Uhlenbeck, Regularity for a class of nonlinear elliptic systems, Acta Math. 138 (1977), 219-240.

[86] M.-T. Wang, Interior gradient bounds for solutions to the minimal surface system, Am. J. Math. 126 (2004), 921-934.

[87] M.-T. Wang, The Dirichlet problem for the minimal surface system in arbitrary dimensions and codimensions, Commun. Pure Appl. Math. 57 (2004), 267-281.

[88] B. White, Existence of least-area mappings of N-dimensional domains, Ann. Math. (2) 118 (1983), 179-185.

[89] K. Yosida, Functional analysis, 6th ed., Springer, Berlin 1980.

SNS Pisa, Piazza dei Cavalieri 7, 56126 Pisa, Italy

e-mail: lisa.beck@sns.it

Department Mathematik, FAU Erlangen-Nürnberg, Bismarckstr. 1 $\frac{1}{2}$, 91054 Erlangen, Germany

e-mail: schmidt@mi.uni-erlangen.de

Eingegangen 23. August 2010, in revidierter Fassung 19. April 2011 